\title{
SEMICLASSICAL EVOLUTION WITH LOW REGULARITY
}

\author{
FRANÇOIS GOLSE AND THIERRY PAUL
}

\begin{abstract}
We prove semiclassical estimates for the Schrödinger-von Neumann evolution with $C^{1,1}$ potentials and density matrices whose square root have either Wigner functions with low regularity independent of the dimension, or matrix elements between Hermite functions having long range decay. The estimates are settled in different weak topologies and apply to initial density operators whose square root have Wigner functions 7 times differentiable, independently of the dimension. They also apply to the $N$ body quantum dynamics uniformly in $N$. In a appendix, we finally estimate the dependence in the dimension of the constant appearing on the Calderon-Vaillancourt Theorem.
\end{abstract}

\section{Contents}

1. Introduction 1

2. Main results 5

3. Semiclassical Wasserstein 11

4. Proofs of Theorems 2.1, 2.2 and 2.3 13

5. Proof of Theorem 4.1 item (III) 14

6. Proof of Theorem 4.1 item (II) 17

6.1. Construction of $T_{F}^{-\frac{1}{2}} \quad 17$

6.2. Hypothesis (1) in Theorem $2.2 \Longrightarrow\left(1^{\prime}\right)$ in Lemma $6.1 \quad 21$

6.3. Hypothesis (1) and (2) in Theorem $2.2 \Longrightarrow\left(2^{\prime}\right)$ in Lemma 6.1

7. Proof of Theorem 4.1 item (I) 24

7.1. Hermite as exact Gaussian quasimodes 24

7.2. Weyl calculus through Wigner operators 26

7.3. The case $d=1 \quad 28$

7.4. The case of any dimension $d \quad 34$

7.5. End of the proof of item (I) in Theorem 4.1 35

8. The case of $N$ particles $\quad 35$

Appendix A. Proof of the triangle inequality 37

Appendix B. Quantum Wasserstein and weak topologies 43

B.1. $L^{2}$ test functions 43

B.2. $\quad L^{\infty}$ test functions 46

Appendix C. An estimation of the Calderon-Vaillancourt constant 49

References $\quad 54$

\section{INTRODUCTION}

The semiclassical approximation links the quantum dynamics of Hamiltonian, say, $H=-\frac{\hbar^{2}}{2} \Delta+V(x)$ on $L^{2}\left(\mathbf{R}^{d}, d x\right)$, to the underlying classical one, namely the flow generated by the Hamiltonian $h(p, q)=\frac{1}{2} p^{2}+V(q)$ on $\mathbf{R}^{2 d} \simeq T^{*} \mathbf{R}^{d}$. This quite indirect 
link, particularly efficient when the Planck constant takes small values, relies on the presence of fast oscillations in the initial data whose speed diverges linearly in $\hbar^{-1}$ as $\hbar \rightarrow 0$.

To our knowledge, all results in semiclassical approximations are subjects to the following alternative:

- either no specific ansatz is made for the initial data of the quantum dynamics. In that case, under some tightness conditions of the initial data, revealing the size of oscillations, and along subsequences of values of $\hbar$ tending to 0 , the Wigner function (or equivalently the Husimi one) of the solution of the quantum evolution is shown to tend to a solution of the Liouville classical equation, with no estimate of the rate of convergence provided (see, e.g., $[8,9,16]$, and also $[1,6]$ for an extension to potentials whose gradient has only $B V$ regularity)).

Let us recall that, by quantum and classical evolution, we mean the content of the following table:

quantum $\begin{cases}i \hbar \partial_{t} \psi=H \psi, & \psi \in L^{2}\left(\mathbf{R}^{d},\right) \\ \frac{d}{d t} R=\frac{1}{i \hbar}[H, R], & R>0, \text { trace } R=1,\end{cases}$

classical $\left\{\begin{array}{lll}\left(\begin{array}{c}\dot{p} \\ \dot{q}\end{array}\right)=\left(\begin{array}{cc}-\partial_{q} h(p, q) \\ \partial_{p} h(p, q)\end{array}\right), & (p, q) \in T^{*} \mathbf{R}^{d} & \text { Hamilton } \\ \frac{d}{d t} \rho=\{h, \rho\}, & \rho>0, \int_{\mathbf{R}^{2 d}} \rho=1 & \text { Liouville }\end{array}\right.$

- or very precise estimates of the rate of convergence are obtained after some ansatz is made on the initial quantum data (WKB, coherent states for the Schrödinger equation; various type of quantization (pseudodifferential, Weyl, Töplitz calculus) for the von Neumann equation). In this case a "quasimode" is constructed, e.g. a solution of an approximate equation, and the unitarity of the quantum flow (supposed a priori) provides a remainder estimate of order $\hbar^{1 / 2}$ or $\hbar$ in $L^{2}\left(\mathbf{R}^{d}\right)$ or $\mathcal{L}\left(L^{2}\left(\mathbf{R}^{d}\right)\right)$ topology. Let us remark that, although the quasimode is constructed trough the solution of the equations of the classical paradigm, the estimate of the rate of convergence is settled in the topology of the quantum one.

Let us mention also that the accuracy of the results for the von Neumann equation in the second case of the preceding alternative degrades quite rapidly when the space dimension or the number of particles involved get large. This for two reasons. First, estimating the remainder is done through the so-called Calderon-Vaillancourt Theorem, a result very regularity consuming when the total dimension, namely $d=3 N$ in the case of $N$ particle in three dimensions, increases. Basically $C^{\left[\frac{3 N}{2}\right]}$-differentiability for both the potential and the initial data is required. In addition, the $O(\hbar)$ size of the remainder is degraded by the multiplicative constant $\gamma_{d}$ appearing in CalderonVaillancourt Theorem. We give an estimate of $\gamma_{d}$ in Appendix C (Theorem C.4), as we couldn't find it elsewhere. Certainly not sharp, the result we obtain is $\log \gamma_{d} \sim \frac{11}{4} d \log d$, 
and a value of $\gamma_{d}$ larger than the inverse of the Planck constant $h=2 \pi \hbar \sim 6.626 \times 10^{-34}$ already for $N=4$ in three dimensions.

Recently we found a way of somehow estimating directly the "distance" between quantum and classical objects. Given $R(t)$ and $\rho(t)$, solutions of the von Neumann and Liouville equations respectively, we defined in [12] a positive number $E_{\hbar}(\rho(t), R(t))$ satisfying for all time $t$ a "Gronwall type" estimate

$$
E_{\hbar}(\rho(t), R(t))^{2} \leq e^{\max (1,4 \operatorname{Lip}(\nabla V)) t} E_{\hbar}(\rho(0), R(0))^{2},
$$

where $\operatorname{Lip}(\nabla V)$ denotes the Lipschitz constant of $\nabla V$. The definition of $E_{\hbar}$ is given in Definition 3.2 below, and is a generalization of a quantum analog of the Wasserstein distance of exponent 2 introduced in [11].

Let us remark immediately that (1) is uniform in $\hbar$ and doesn't contain any extra semiclassical error term as appearing in estimates involving quasimodes. e.g. $O\left(\hbar^{\infty}\right)$. Consistently $E_{\hbar}(R(0), \rho(0))$ cannot vanish. In fact

$$
E_{\hbar} \geq \frac{1}{2} d \hbar \text {. }
$$

On the other side, the smallness of $E_{\hbar}(R, \rho)$ has a true meaning thanks to the fact that it almost dominates a distance between $\rho$ and the Husimi function of $R$ : the following bound holds true for any $R, \rho \geq 0$ with trace $R=\int_{\mathbf{R}^{2 d}} \rho d p d q=1$, proven in [12] Theorem 2.42 and recalled in Theorem 3.3 of the present paper,

$$
\operatorname{dist}_{\mathrm{MK}, 2}(\widetilde{W}[R], \rho)^{2} \leq E_{\hbar}(R, \rho)^{2}+\frac{1}{2} d \hbar .
$$

The inequality (3) was proven in [12] Theorem 2.42 and is recalled in Theorem 3.3 of the present paper, the definition of the Wasserstein distance dist $\mathrm{MK}, 2_{2}$ is recalled in (21) and $\widetilde{W}[R]$, the Husimi function of $R$, in (10).

Moreover, $E_{\hbar}$ shares with the notion of distance the following type of triangle inequality, valid for any $R \geq 0$, trace $R=1$ and probability measures $f, f^{\prime}$, see Lemma 3.5 in Section 3,

$$
E_{\hbar}\left(f^{\prime}, R_{\hbar}\right) \leq \operatorname{dist}_{\mathrm{MK}, 2}\left(f^{\prime}, f\right)+E_{\hbar}\left(f, R_{\hbar}\right) .
$$

Since the correspondence between $R$ and $\widetilde{W}[R]$ is one-to-one (see Section 2), formula (1) together with (2) will give an estimate for the semiclassical evolution of $R(t)$ solution of the von Neumann equation, estimate expressed this time in the classical paradigm at the contrary of the quasimode ones. More precisely, we will get this estimates if we are able to find a probability measure $\rho^{\text {in }}$ such that

$$
E_{\hbar}\left(\rho^{i n}, R(0)\right)=o(1) \text { as } \hbar \rightarrow 0 .
$$

This task was achieved in the case where $R(0)$ is a Töplitz operator whose symbol $\rho$ is any probability measure on $\mathbf{R}^{2 d}$ (see Definition in Section 2 below): for all $t \in \mathbf{R}$, Theorem 2.7 in [12] (see also Section 3 of the present article) leads to

$$
\operatorname{dist}_{\mathrm{MK}, 2}(\rho(t), \widetilde{W}[R(t)])^{2} \leq \frac{1}{2}\left(1+e^{\max \left(1,4 \operatorname{Lip}(\nabla V)^{2}\right) t}\right) d \hbar,
$$


Note that this result, being valid for any probability measure type symbol $\rho$ and therefore not requiring any regularity condition, is, at our knowledge, unreachable by usual Töplitz calculus proof.

The goal of the present paper is:

(1) to get rid of the Töplitz ansatz we just mentioned

(2) to find practical efficient conditions for quantum initial data to be semiclassically evolved by the von Neumann equation, without any need of concrete ansatz

(3) to obtain propagation estimates of the form (6) for initial density matrices, the Wigner function of the square root of them having low regularity $C^{7}$, independently of the dimension and the number of particles.

(4) to estimate the semiclassical propagation in some "direct" weak distances instead of in the Wasserstein-Husimi formulation of [12], namely a distance $\delta$ between Wigner functions generated by the dual of a space of test functions, or likewise a distance $d$ between density operators associated to the trace duality of a set of test operators (see definition above in this section).

(5) to get semiclassical estimates uniform in the number of particles involved.

Our results will be developed on three nested levels of generality. The most general one involves the existence of a probability density, linked to the initial condition $R^{\text {in }}$ of the von Neumann equation, satisfying explicit conditions. The second one gives a concrete realization of such a density under explicit conditions on the size of the matrix elements of $\sqrt{R(0)}$ between (semiclassically scaled) Hermite functions. The third one shows that such conditions are satisfied by operators whose Wigner function satisfies low regularity conditions.

Each of these results will be expressed in different forms, using the distance dist ${ }_{\mathrm{MK}, 2}$ or in terms of the following ones, introduced in [10] Appendix B and studied in Appendix B.2 on the present paper.

We recall the definition for two Hilbert-Schmidt (in particular density) operators $R$ and $S$ and $M>0$.

$$
\begin{aligned}
& \delta_{M}\left(W_{\hbar}[R], W_{\hbar}[S]\right)=\sup _{\max _{|\alpha|,|\beta| \leq M}\left\|\partial_{x}^{\alpha} \partial_{\xi}^{\beta} f\right\|_{L^{\infty} \leq 1}}\left|\int\left(W_{\hbar}[R](x, \xi)-W_{\hbar}[S](x, \xi)\right) f(x, \xi) d x d \xi\right|,
\end{aligned}
$$

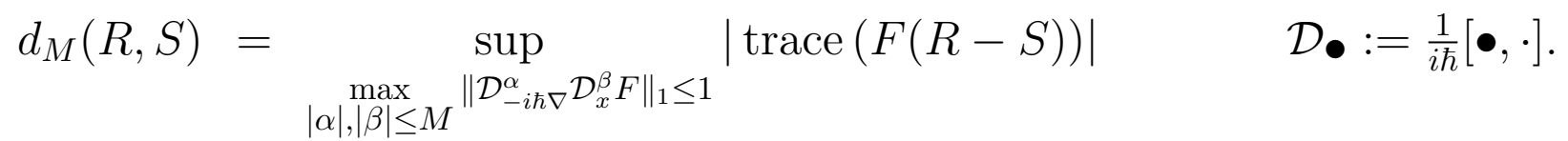

The different "distances" used in this article are nested in the following chain of inequalities (see Propositions B.7 and 3.4) valid for $R, S$ density operators

$d_{2[d / 4]+2}(R, S) \leq 2^{d} \delta_{2[d / 4]+2}\left(W_{\hbar}[R], W_{\hbar}[S]\right) \leq \operatorname{dist}_{\mathrm{MK}, 2}\left(\widetilde{W}_{\hbar}[R], \widetilde{W}_{\hbar}[S]\right)+\frac{2 d \gamma_{d}}{\sqrt{\pi}} \sqrt{\hbar} \leq E_{\hbar}\left(\widetilde{W}_{\hbar}[R], S\right)+\left(\frac{2 d \gamma_{d}}{\sqrt{\pi}}+\frac{d}{2}\right) \sqrt{\hbar}$ 


$$
\begin{aligned}
d_{2[d / 4]+2}(R, S) & \leq 2^{d} \delta_{2[d / 4]+2}\left(W_{\hbar}[R], W_{\hbar}[S]\right) \\
\leq \operatorname{dist}_{\mathrm{MK}, 2}\left(\widetilde{W}_{\hbar}[R], \widetilde{W}_{\hbar}[S]\right)+\frac{2 d \gamma_{d}}{\sqrt{\pi}} \sqrt{\hbar} & \leq E_{\hbar}\left(\widetilde{W}_{\hbar}[R], S\right)+\left(\frac{2 d \gamma_{d}}{\sqrt{\pi}}+\frac{d}{2}\right) \sqrt{\hbar}
\end{aligned}
$$

where $\gamma_{d}$ is the constant that appears in the Calderon-Vaillancourt Theorem C.4 below.

We will use finally a last distance $\delta$ whose definition, independent of the dimension and involving $L^{2}$ test functions is the following (once again $R$ and $S$ are Hilbert-Schmidt operators).

$$
\left.\delta\left(W_{\hbar}[R], W_{\hbar}[S)\right]\right)=\sup _{\substack{\operatorname{Lip}(f) \leq 1 \\\|f\|_{L^{2}\left(\mathbf{R}^{2 d}\right)},\|\nabla f\|_{L^{2}\left(\mathbf{R}^{2 d}\right)} \leq 1}}\left|\int\left(W_{\hbar}[R]-W_{\hbar}[S]\right) f(x, \xi) d x d \xi\right| .
$$

The series of nested links between $\delta$, dist ${ }_{\mathrm{MK}, 2}$ and $E_{\hbar}$, this time independent of the dimension, is the following (see Section B.1 in Appendix B below), for $R, S$ density operators.

$\delta\left(\widetilde{W}_{\hbar}[R], \widetilde{W}_{\hbar}[S]\right) \leq \operatorname{dist}_{\mathrm{MK}, 2}\left(\widetilde{W}_{\hbar}[R], \widetilde{W}_{\hbar}[S]\right)+\sqrt{\hbar}\left\|W_{\hbar}[R-S]\right\|_{L^{2}} \leq \sqrt{2} E_{\hbar}\left(\widetilde{W}_{\hbar}[R], S\right)+\sqrt{\hbar}\left\|W_{\hbar}[R-S]\right\|_{L^{2}}$.

In our last main result, Theorem 8.2, the distance $\delta$ will provide a topology for the $N$-body semiclassical propagation of factorized (e.g. bosonic) initial data which will be uniform in $N$ as $N \rightarrow \infty$.

The three type of results mentioned earlier are expressed in Section 2 by Theorems 2.1, 2.2 and 2.3 respectively, in reverse order of nesting for pedagogical purposes. Their proofs are given in Section 4 by showing that they are mostly corollaries of the three items of Theorem 4.1 proven in Sections 5, 6 and 7 respectively. Section 3 is devoted to the quantum analogue of the Wasserstein distance of exponent two and its properties. Semiclassical uniform in $N$ estimates for the $N$-body quantum propagation are stated and proved in Section 8 and the three appendices A, B and $\mathrm{C}$ are devoted respectively to the proof of the triangle type inequality for $E_{\hbar}$ just mentioned, the comparison of $E_{\hbar}$ with weak topologies and an estimation of the Calderon-Vaillancourt constant.

\section{MAIN RESULTS}

For any real function $V$ of class $C^{1,1}$ on $\mathbf{R}^{d}$ such that the operator $-\frac{1}{2} \hbar^{2} \Delta+V$ is essentially self-adjoint on $L^{2}\left(\mathbf{R}^{d}\right)$, we consider the von Neumann equation

$$
i \hbar \frac{d}{d t} R_{\hbar}(t)=\left[-\frac{1}{2} \hbar^{2} \Delta+V, R_{\hbar}(t)\right], R_{\hbar}(0)=R^{i n}
$$

where the initial condition $R^{i n}$ is a density matrix, that is that $R^{i n}$ is positive and trace $R^{i n}=1$.

Obviously

$$
R^{i n}=A^{2}, \quad A \text { being a positive Hilbert-Schmidt operator. }
$$


Let us recall the definition of the Wigner and Husimi transforms of a density operator on $\mathfrak{H}$ (see e.g. [16] or [11] Appendix B for a short review). If $R$ is the density matrix of integral kernel $r$, its Wigner transform at scale $\hbar$ is the function on $\mathbf{R}^{d} \times \mathbf{R}^{d}$ defined by the formula

$$
W_{\hbar}[R](x, \xi):=\frac{1}{(2 \pi)^{d}} \int_{\mathbf{R}^{d}} e^{-i \xi \cdot y} r\left(x+\frac{1}{2} \hbar y, x-\frac{1}{2} \hbar y\right) d y .
$$

The Husimi transform of $R$ is

$$
\widetilde{W}_{\hbar}[R]:=e^{\hbar \Delta_{x, \xi} / 4} W_{\hbar}[R]
$$

and we recall that

$$
\widetilde{W}_{\hbar}[R] \geq 0, \int_{\mathbf{R}^{d} \times \mathbf{R}^{d}} \widetilde{W}_{\hbar}[R](x, \xi) d x d \xi=\int_{\mathbf{R}^{d} \times \mathbf{R}^{d}} W_{\hbar}[R](x, \xi) d x d \xi=1 .
$$

In particular, $\widetilde{W}_{\hbar}[R]$ is a probability density on $\mathbf{R}^{d} \times \mathbf{R}^{d}$ for each $R \in \mathcal{D}\left(L^{2}\left(\mathbf{R}^{d}\right)\right)$.

Moreover, by Remark 2.3. in $[12], \widetilde{W}_{\hbar}[R]$ determines $R$ uniquely.

We denote by $\rho(t)$ the solution of the following Liouville equation on $\mathbf{R}^{2 d}$ with initial condition $\widetilde{W}\left[R^{i n}\right]$ :

$$
\dot{\rho}=\left\{\frac{1}{2} \xi^{2}+V(x), \rho\right\}, \quad \rho(0)=\widetilde{W}\left[R^{i n}\right]
$$

We denote also by $\Phi^{t}$ the Hamiltonian flow of Hamiltonian $\frac{1}{2} p^{2}+V(q)$ so that

$$
\rho(t)=\rho(0) \circ \Phi^{-t} \text {. }
$$

Moreover we define

$$
\lambda=\frac{1+\max \left(4 \operatorname{Lip}(\nabla V)^{2}, 1\right)}{2} .
$$

Finally we define $\mathcal{R}(t)$ as the operator whose Wigner function is $\rho(t)$ :

$$
W_{\hbar}[\mathcal{R}(t)]:=\rho(t) .
$$

In the sequel we will denote

- by $z=(x, \xi)$ a point in $T^{*} \mathbf{R}^{d}$,

- by $\mathcal{P}\left(\mathbf{R}^{2 d}\right)$ the set of probability densities on $T^{*} \mathbf{R}^{d}$

- by $\mathcal{P}_{2}\left(\mathbf{R}^{2 d}\right)$ the set of probability densities on $T^{*} \mathbf{R}^{d}$ with finite second moments

- by $\mathcal{D}\left(L^{2}\left(\mathbf{R}^{d}\right)\right)$ the set of density matrices on $L^{2}\left(\mathbf{R}^{d}\right)$

- by $\mathcal{D}_{2}\left(L^{2}\left(\mathbf{R}^{d}\right)\right)$ the set of density matrices $R$ on $L^{2}\left(\mathbf{R}^{d}\right)$ satisfying

$$
\operatorname{trace}\left(-\hbar^{2} \Delta+x^{2}\right) R<\infty
$$


For $k=\left(k_{1}, \ldots, k_{d}\right) \in \mathbf{Z}^{d}$ we define: the Fourier coefficients $a_{k}(z)$ of a function $a\left(z_{1}, \ldots, z_{d}\right), z_{i} \in T^{*} \mathbf{R}$ by

$$
a_{k}(z)=\int_{\mathbf{T}^{d}} a\left(z_{1} e^{i k_{1} \theta_{1}}, \ldots, z_{d} e^{i k_{d} \theta_{d}}\right) e^{i k \cdot \theta} d \theta:=\int_{\mathbf{T}^{d}} a\left(e^{i \theta} z\right) e^{i k \theta} d \theta .
$$

Definition. For $K, M \in \mathbf{R}^{+}, N \in \mathbf{N}$, we define on $L^{1}\left(\mathbf{R}^{2 d}\right)$ the norm

(15) $\|a\|_{M, K, N}:=\sup _{k \in \mathbf{Z}^{d}} \sup _{z \in \mathbf{R}^{2 d}} \prod_{l=1}^{d}\left(\left|z_{l}\right|^{2}+1\right)^{M}\left(\left|k_{l}\right|+d\right)^{K} \sup _{\left|\alpha_{1}\right|, \ldots,\left|\alpha_{d}\right| \leq N}\left|\prod_{m=1}^{d}\left(\sqrt{\hbar} D_{z_{m}}\right)^{\alpha_{m}} a_{k}(z)\right|$,

Theorem 2.1. Let $R(t)$ be the solution of the von Neumann equation (7) with initial condition $R^{\text {in }}$ and $\mathcal{R}(t)$ the operator whose Wigner function solves the Liouville equation (11) with initial data the Husimi function of $R^{i n}$. Let $\delta$ the distance defined in Definition B.1.

Let us suppose that there exist two functions $\mu(\hbar), \nu(\hbar)$ satisfying

$$
\sqrt{\hbar} \mu(\hbar)=o(1), \mu(\hbar) \nu(\hbar)=o(1) \text { as } \hbar \rightarrow 0,
$$

such that, for some $\epsilon \in] 0,1[$,

(1) $\left\|W_{\hbar}\left[\sqrt{R^{i n}}\right]\right\|_{\frac{3}{4}+\epsilon, \frac{7}{2}+3 \epsilon, 3} \leq(2 \pi \hbar)^{-\frac{d}{2}} \mu(\hbar)$

(2) $\hbar^{\frac{1}{2}}\left\|W_{\hbar}\left[\sqrt{R^{i n}}\right]\right\|_{\frac{3}{4}+\epsilon, \frac{5}{2}+3 \epsilon, 4} \leq(2 \pi \hbar)^{-\frac{d}{2}} \nu(\hbar)$

Then, for all $t \in \mathbf{R}$,

$$
\operatorname{dist}_{\mathrm{MK}, 2}\left(\widetilde{W}\left[R_{\hbar}(t)\right], \rho(t)\right) \leq D_{I} e^{\lambda|t|} \max (\sqrt{\hbar}, \sqrt{\hbar} \mu(\hbar), \sqrt{\mu(\hbar) \nu(\hbar)}) .
$$

Moreover, if $W\left[R_{\hbar}^{i n}\right] \in L^{1}\left(\mathbf{R}^{2 d}\right)$ for each $\left.\hbar \in\right] 0,1[$, we have

$$
\begin{aligned}
& \left.2^{-d} d_{2[d / 4]+3}(R(t), \mathcal{R}(t)) \leq \delta_{2[d / 4]+3}\left(W_{\hbar}[R(t))\right], W_{\hbar}\left[R^{i n}\right] \circ \Phi^{-t}\right) \\
& \leq D_{I} e^{\lambda|t|} \max (\sqrt{\hbar}, \sqrt{\hbar} \mu(\hbar), \sqrt{\mu(\hbar) \nu(\hbar)})+\sqrt{\hbar}\left(\frac{2 d \gamma_{d}}{\sqrt{\pi}}+e^{1+\operatorname{Lip}(\nabla V))|t|)}\left\|W_{\hbar}\left[R^{i n}\right]\right\|_{L^{1}\left(\mathbf{R}^{2 d}\right)}\right)
\end{aligned}
$$

Here $D_{I}$ is given in (90).

Using (23) and taking $\mu(\hbar)=1, \nu(\hbar)=\hbar^{\frac{1}{2}}$ so that $\sqrt{\mu(\hbar) \nu(\hbar)}=\hbar^{\frac{1}{4}}$, we derive easily the following (maybe more tractable) corollary.

Corollary Let $R_{\hbar}$ be a family of density matrices of $L\left(\mathbf{R}^{d}\right)$. Let us assume that the Wigner function $W_{\hbar}\left[\sqrt{R^{i n}}\right](x, \xi)$ of $\sqrt{R^{\text {in }}}$ has derivatives up to order $\frac{13}{2}+3 \epsilon$ bounded 


$$
\begin{aligned}
& \text { by } C(2 \pi \hbar)^{-\frac{d}{2}}\left(\left(\xi^{2}+x^{2}\right)+d\right)^{-\frac{10}{4}-4 \epsilon}, C>0 \text {, i.e. } \\
& \sup _{\left|\beta_{1}\right|, \ldots,\left|\beta_{d}\right| \leq 7}\left|\prod_{m=1}^{d} D_{(x, \xi)}^{\beta_{m}} W_{\hbar}\left[\sqrt{R^{i n}}\right](x, \xi)\right| \leq \frac{C(2 \pi \hbar)^{-\frac{d}{2}}}{\left(\left(\xi^{2}+x^{2}\right)^{2}+d\right)^{\frac{10}{4}+3 \epsilon}} \quad \forall(x, \xi) \in \mathbf{R}^{2 d} .
\end{aligned}
$$

Then there exist $D, D^{\prime}$ such that, for all $t \in \mathbf{R}$,

$$
\operatorname{dist}_{M K, 2}\left(\widetilde{W}\left[R_{\hbar}(t)\right], \rho(t)\right) \leq D \hbar^{\frac{1}{4}} e^{\lambda|t|}
$$

Moreover, if, in addition, $\left\|W_{\hbar}\left[R^{i n}\right]\right\|_{L^{1}\left(\mathbf{R}^{2 d}\right)} \leq C^{\prime}<\infty$, for each $\hbar \in[0,1]$, we have

$$
\left.\delta_{2[d / 4]+3}\left(W_{\hbar}[R(t))\right], W_{\hbar}\left[R^{i n}\right] \circ \Phi^{-t}\right) \leq D^{\prime} \hbar^{\frac{1}{4}} e^{2 \lambda|t|} .
$$

Let us make a few remarks concerning Theorem 2.1.

The factor $(2 \pi \hbar)^{-\frac{d}{2}}$ in Theorem 2.1 and its corollary might seem strange, but it is in fact natural, if we think that the Wigner function of $\left(\sqrt{R^{i n}}\right)^{2}$ has to be the one of $R^{i n}$, a density operator.

Indeed if we think that the Wigner function of an operator is the quotient of its Weyl symbol by $(2 \pi \hbar)^{d}$, we have, using the Moyal product $\star_{\text {Moyal }}$, that

$$
(2 \pi \hbar)^{d} W_{\hbar}\left[R^{i n}\right]=(2 \pi \hbar)^{d} W_{\hbar}\left[\sqrt{R^{i n}}\right] \star_{\text {Moyal }}(2 \pi \hbar)^{d} W_{\hbar}\left[\sqrt{R^{i n}}\right.
$$

so that

$$
W_{\hbar}\left[R^{i n}\right]=\left((2 \pi \hbar)^{\frac{d}{2}} W_{\hbar}\left[\sqrt{R^{i n}}\right]\right) \star_{\text {Moyal }}\left((2 \pi \hbar)^{\frac{d}{2}} W_{\hbar}\left[\sqrt{R^{i n}}\right) .\right.
$$

In order to directly implement semiclassical approximation in weak sense, other than the results presented in the introduction obtained by compactness methods without rate of convergence, one should work with Wigner or Husimi functions. But then one faces the difficulty of the non positiveness of the Wigner function. Of course, one can cure this default by using instead the Husimi function, but the Husimi function follows an evolution equation involving analyticity regularity (see [2]).

Therefore it seems to us that the only way of obtaining precise weak semiclassical results by "standard" methods consists in constructing quasimodes in strong topology, a way obviously very regularity consuming. On the contrary, the results in the present paper uses weak topology from the beginning, and allows us to obtain results requiring little regularity independent of the dimension.

Let us mention finally that we studied in [13], section 5, the pertinence of weak versus strong topologies concerning the transition between quantum and classical paradigms.

Actually the content of Theorem 2.1 is a particular case of the following more general result. 
Let us define the Hermite orthonormal basis of $L^{2}\left(\mathbf{R}^{d}\right)$ as $\left\{H_{j}, j \in \mathbf{N}^{d}\right\}$, i.e., for $j=\left(j_{1}, \ldots, j_{d}\right)$,

$$
H_{j}=h_{j_{1}} \otimes \cdots \otimes h_{j_{d}} \text { where }\left(-\hbar^{2} \frac{d^{2}}{d x^{2}}+x^{2}\right) h_{k}=(2 k+1) h_{k},\left\|h_{k}\right\|_{L^{2}(\mathbf{R})}=1 .
$$

We will denote by $(\cdot, \cdot)$ the scalar product in $L^{2}\left(\mathbf{R}^{d}\right)$.

Theorem 2.2. Let us suppose that there exist two functions $\mu^{\prime}(\hbar), \nu^{\prime}(\hbar)$ satisfying

$$
\sqrt{\hbar} \mu^{\prime}(\hbar)=o(1), \mu^{\prime}(\hbar) \nu^{\prime}(\hbar)=o(1) \text { as } \hbar \rightarrow 0,
$$

such that, for some $\epsilon \in] 0,1[$,

(1) $\left|\left(H_{i}, \sqrt{R^{i n}} H_{j}\right)\right| \leq(2 \pi \hbar)^{\frac{d}{2}} \mu^{\prime}(\hbar) \prod_{1 \leq l \leq d}\left|\hbar j_{l}+\frac{1}{2}\right|^{-\frac{3}{4}-\epsilon}\left(\left|i_{l}-j_{l}\right|+1\right)^{-2-\epsilon}$,

(2) $\sup _{O \in \Omega_{1}}\left|\left(H_{i},\left[O, \sqrt{R^{i n}}\right] H_{j}\right)\right| \leq(2 \pi \hbar)^{\frac{d}{2}} \nu^{\prime}(\hbar) \prod_{1 \leq l \leq d}\left|\hbar j_{l}+\frac{1}{2}\right|^{-\frac{1}{2}-\epsilon}\left(\left|i_{l}-j_{l}\right|+1\right)^{-1-\epsilon}$,

$$
\text { where } \Omega_{1}=\left\{y_{j}, \pm \hbar \partial_{y_{j}} \text { on } L^{2}\left(\mathbf{R}^{d}, d y\right), j=1, \ldots, d\right\} \text {. }
$$

Then, for all $t \in \mathbf{R}$,

$$
\operatorname{dist}_{M K, 2}\left(\widetilde{W}\left[R_{\hbar}(t)\right], \rho(t)\right) \leq D_{I I} e^{\lambda|t|} \max \left(\sqrt{\hbar}, \sqrt{\hbar} \mu^{\prime}(\hbar), \sqrt{\mu^{\prime}(\hbar) \nu^{\prime}(\hbar)}\right) .
$$

Moreover, if $W\left[R_{\hbar}^{i n}\right] \in L^{1}\left(\mathbf{R}^{2 d}\right)$ for each $\left.\hbar \in\right] 0,1[$, we have

$$
\begin{aligned}
& \left.2^{-d} d_{2[d / 4]+3}(R(t), \mathcal{R}(t)) \leq \delta_{2[d / 4]+3}\left(W_{\hbar}[R(t))\right], W_{\hbar}\left[R^{i n}\right] \circ \Phi^{-t}\right) \\
& \leq D_{I I} e^{\lambda|t|} \max \left(\sqrt{\hbar}, \sqrt{\hbar} \mu^{\prime}(\hbar), \sqrt{\mu^{\prime}(\hbar) \nu^{\prime}(\hbar)}\right) .+\sqrt{\hbar}\left(\frac{2 d \gamma_{d}}{\sqrt{\pi}}+e^{1+\operatorname{Lip}(\nabla V))|t|)}\left\|W_{\hbar}\left[R^{i n}\right]\right\|_{L^{1}\left(\mathbf{R}^{2 d}\right)}\right)
\end{aligned}
$$

Here $D_{I I}$ is given in (50).

\section{Remark}

Here also the factor $(2 \pi \hbar)^{d}$ is natural. For example, thanks to it, estimate (1) gives that trace $R^{\text {in }}$ is finite.

Once again, Theorem 2.2 is also a particular case of a more general one. Let us first set up the following definition (see [11], Appendix B for further details).

Definition [Töplitz operators] For each $z=x+i \xi \in \mathbf{C}^{d}$, we denote

$$
|z, \hbar\rangle: y \mapsto(\pi \hbar)^{-d / 4} e^{-|y-x|^{2} / 2 \hbar} e^{i \xi \cdot(y-x) / \hbar} \quad \||z, \hbar\rangle \|_{L^{2}\left(\mathbf{R}^{d}\right)}=1,
$$

and we designate by $|z, \hbar\rangle\langle z, \hbar|$ the orthogonal projection on the line $\mathbf{C}|z, \hbar\rangle$ in $\mathfrak{H}$. For each Borel probability density $\mu$ on $\mathbf{R}^{d} \times \mathbf{R}^{d}$, we define the Töplitz operator of symbol $\mu$ as the operator defined weakly on $L^{2}\left(\mathbf{R}^{d}\right)$ by the formula

$$
\mathrm{Op}_{\hbar}^{T}(\mu):=\frac{1}{(2 \pi \hbar)^{d}} \int_{\mathbf{R}^{d} \times \mathbf{R}^{d}}|x+i \xi, \hbar\rangle\langle x+i \xi, \hbar| \mu(x, \xi) d x d \xi .
$$


One easily shows that

$$
\operatorname{trace} \mathrm{Op}_{\hbar}^{T}\left((2 \pi \hbar)^{d} \mu\right)=1
$$

so that $\mathrm{Op}_{\hbar}^{T}\left((2 \pi \hbar)^{d} \mu\right)$ is trace class and admits an $L^{2}$ spectral decomposition

$$
\mathrm{Op}_{\hbar}^{T}\left((2 \pi \hbar)^{d} \mu\right)=\sum_{i \in \mathbf{N}} \mu_{i}\left|\psi_{i}\right\rangle\left\langle\psi_{i}\right|, \quad \psi_{i} \in L^{2}\left(\mathbf{R}^{d}\right)
$$

When $F>0, \mathrm{Op}_{\hbar}^{T}\left((2 \pi \hbar)^{d} F\right)$ is injective, as any element $\psi$ of its kernel would satisfy $\langle x, \xi \mid \psi\rangle=0$ for almost all $(x, \xi) \in \mathbf{R}^{2 d}$ which would imply $\psi=0$ by completeness of discrete families of coherent states (see [3]). So $\mathrm{Op}_{\hbar}^{T}\left((2 \pi \hbar)^{d} \mu\right]^{-1 / 2}$ exists as a unbounded operator on $L^{2}\left(\mathbf{R}^{d}\right)$ as defined by the spectral theorem

$$
\mathrm{Op}_{\hbar}^{T}\left((2 \pi \hbar)^{d} F\right)^{-1 / 2}=\sum_{i \in \mathbf{N}} \mu_{i}^{-1 / 2}\left|\psi_{i}\right\rangle\left\langle\psi_{i}\right|
$$

on the domain $\mathcal{D}=\left\{\psi \in L^{2}\left(\mathbf{R}^{d}\right), \sum_{i \in \mathbf{N}} \mu_{i}^{-1}\left|\left\langle\psi_{i} \mid \psi\right\rangle\right|^{2}<\infty\right\}$.

Theorem 2.3. Let $R_{\hbar}^{i n}=R^{i n}$ be a family of density matrices on $L^{2}\left(\mathbf{R}^{d}\right)$ satisfying the following hypothesis

\section{(1) (tightness)}

there exists a probability density $F>0$ on $\mathbf{R}^{2 d}$ satisfying $\int(|x|+|\xi|) F(d x, d \xi)<\infty$ such that, for each $\hbar \in(0,1]$,

$\mathrm{Op}_{\hbar}^{T}\left((2 \pi \hbar)^{d} F\right)^{-1 / 2} \sqrt{R^{i n}}, \sqrt{R^{i n}} \mathrm{Op}_{\hbar}^{T}\left((2 \pi \hbar)^{d} F\right)^{-1 / 2}$ have bounded extensions to $L^{2}\left(\mathbf{R}^{d}\right)$

(we will denote by the same symbols the operators and their bounded extensions).

(2) (semiclassical hypothesis)

there exists $\tau(\hbar)=o(1)$ as $\hbar \rightarrow 0$ such that

$$
\sup _{O \in \Omega}\left\|\mathrm{Op}_{\hbar}^{T}\left((2 \pi \hbar)^{d} F\right)^{-1 / 2} \sqrt{R^{i n}}\left[O, \sqrt{R^{i n}} \mathrm{Op}_{\hbar}^{T}\left((2 \pi \hbar)^{d} F\right)^{-1 / 2}\right]\right\|=\tau(\hbar),
$$

where $\Omega=\left\{-\hbar^{2} \Delta_{y}+|y|^{2}, y_{j}, \pm \hbar \partial_{y_{j}}\right.$ on $\left.L^{2}\left(\mathbf{R}^{d}, d y\right), j=1, \ldots, d\right\}$.

Let $R_{\hbar}(t)$ the solution of the von Neumann equation $(7)$ and $\rho(t)$ the solution of the Liouville equation (11) on $\mathbf{R}^{2 d}$ with initial condition $\widetilde{W}\left[R^{i n}\right]$.

Then, for all $t \in \mathbf{R}$,

$$
E_{\hbar}(\rho(t), R(t)) \leq \frac{D_{I I I}}{\sqrt{2}} e^{\lambda|t|} \max (\sqrt{\hbar}, \sqrt{\tau(\hbar)})
$$

and therefore

$$
\operatorname{dist}_{\mathrm{MK}, 2}\left(\widetilde{W}_{\hbar}[R(t)], \rho(t)\right) \leq D_{I I I} e^{\lambda|t|} \max (\sqrt{\hbar}, \sqrt{\tau(\hbar)}),
$$

with $D_{I I I}$ given by $(28)$. 


\section{Remark 2.4.}

Hypothesis (1) is not empty as $R_{\hbar}:=\mathrm{Op}_{\hbar}^{T}\left((2 \pi \hbar)^{d} F\right)$ satisfies obviously (1).

When the tightness bound in (1) is uniform in $\hbar,(2)$ can be replaced by

$\sup _{O \in \Omega}\left\|\left[O, R_{\hbar}^{1 / 2} \mathrm{Op}_{\hbar}^{T}\left((2 \pi \hbar)^{d} F\right)^{-1 / 2}\right]\right\|=o(1)$.

$O \in \Omega$

\section{Remark 2.5.}

The condition (1) implies that $\mathrm{Op}_{\hbar}^{T}\left((2 \pi \hbar)^{d} F\right)^{-1 / 2} R_{\hbar} \mathrm{Op}_{\hbar}^{T}\left((2 \pi \hbar)^{d} F\right)^{-1 / 2}$ is bounded. Therefore, writing the spectral decomposition $\mathrm{Op}_{\hbar}^{T}\left((2 \pi \hbar)^{d} F\right)=\sum_{j \in \mathbf{N}} \lambda_{j}|j\rangle\langle j|$,

$$
\left\langle j\left|R_{\hbar}\right| j\right\rangle=O\left(\lambda_{j}\right) \text { so that }\left\langle j\left|R_{\hbar}\right| j\right\rangle \rightarrow 0 \text { as } j \rightarrow \infty,
$$

since $\mathrm{Op}_{\hbar}^{T}\left((2 \pi \hbar)^{d} F\right)$ is trace class so that $\lambda_{j} \rightarrow 0$ as $j \rightarrow \infty$.

Moreover, since $F$ and therefore $\mathrm{Op}_{\hbar}^{T}\left((2 \pi \hbar)^{d} F\right)$ have finite moments, the eigenstates $|j\rangle$ must be phase-space localized at infinity as $j \rightarrow \infty$ and condition (1) reflects the lack of concentration of $R_{\hbar}$ at infinity.

\section{Semiclassical Wasserstein}

Let us start this section by recalling the definition of the second order Wasserstein

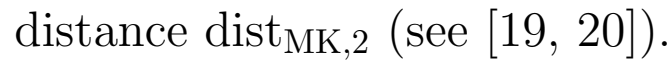

The Wasserstein distance of order two between two probability measures $\mu, \nu$ on $\mathbf{R}^{m}$ with finite second moments is defined as

$$
\operatorname{dist}_{\mathrm{MK}, 2}(\mu, \nu)^{2}=\inf _{\gamma \in \Gamma(\mu, \nu)} \int_{\mathbf{R}^{m} \times \mathbf{R}^{m}}|x-y|^{2} \gamma(d x, d y)
$$

where $\Gamma(\mu, \nu)$ is the set of probability measures on $\mathbf{R}^{m} \times \mathbf{R}^{m}$ whose marginals on the two factors are $\mu$ and $\nu$, i.e., for ny test function $a$,

$$
\int_{\mathbf{R}^{m} \times \mathbf{R}^{m}} a(x) \gamma(d x, d y)=\int_{\mathbf{R}^{m}} a(x) \mu(d x), \int_{\mathbf{R}^{m} \times \mathbf{R}^{m}} a(y) \gamma(d x, d y)=\int_{\mathbf{R}^{m}} a(y) \nu(d y) .
$$

Let us define now the notion of coupling between classical and quantum densities.

Definition 3.1. Let $p \equiv p(x, \xi)$ be a probability density on $\mathbf{R}^{d} \times \mathbf{R}^{d}$, and let $R \in \mathcal{D}(\mathfrak{H})$. $A$ coupling of $p$ and $R$ is a measurable function $Q:(x, \xi) \mapsto Q(x, \xi)$ defined a.e. on $\mathbf{R}^{d} \times \mathbf{R}^{d}$ and with values in $\mathcal{L}(\mathfrak{H})$ s.t. $Q(x, \xi) \in \mathcal{D}(\mathfrak{H})$ for a.e. $(x, \xi) \in \mathbf{R}^{d} \times \mathbf{R}^{d}$, and

$$
\left\{\begin{array}{l}
\operatorname{trace}(Q(x, \xi))=p(x, \xi) \text { for a.e. }(x, \xi) \in \mathbf{R}^{d} \times \mathbf{R}^{d}, \\
\iint_{\mathbf{R}^{d} \times \mathbf{R}^{d}} Q(x, \xi) d x d \xi=R .
\end{array}\right.
$$

The set of all such functions is denoted by $\mathcal{C}(p, R)$.

Mimicking the definition of Monge-Kantorovich distances, we next define the pseudodistance between $R_{\hbar}$ and $f$ in terms of an appropriate "cost function" analogous to the quadratic cost function used in optimal transport. 
Definition 3.2. For each probability density $p \equiv p(x, \xi)$ on $\mathbf{R}^{d} \times \mathbf{R}^{d}$ and each $\rho \in \mathcal{D}(\mathfrak{H})$, we set

$$
E_{\hbar}(p, R):=\left(\inf _{Q \in \mathcal{C}(p, R)} \int_{\mathbf{R}^{d} \times \mathbf{R}^{d}} \operatorname{trace}\left(c_{\hbar}(x, \xi) Q(x, \xi)\right) d x d \xi\right)^{1 / 2} \in[0,+\infty],
$$

where the transport cost $c_{\hbar}$ is the function of $(x, \xi)$ with values in the set of unbounded operators on $\mathfrak{H}=L^{2}\left(\mathbf{R}_{y}^{d}\right)$ defined by the formula

$$
c_{\hbar}(x, \xi):=\frac{1}{2}\left(|x-y|^{2}+\left|\xi+i \hbar \nabla_{y}\right|^{2}\right) .
$$

Immediate properties of $E_{\hbar}$ are stated in the following result.

Theorem 3.3. [from Theorem 2.4 in [12]]

(1) For each probability density $p$ on $\mathbf{R}^{d} \times \mathbf{R}^{d}$ such that $\int_{\mathbf{R}^{d} \times \mathbf{R}^{d}}\left(|x|^{2}+\left.\xi\right|^{2}\right) p(x, \xi) d x d \xi<\infty$ and each $R \in \mathcal{D}(\mathfrak{H})$, one has

$$
E_{\hbar}(p, R)^{2} \geq \frac{1}{2} d \hbar .
$$

(2) Let $R_{\hbar}=\mathrm{Op}_{\hbar}^{T}\left((2 \pi \hbar)^{d} \mu\right)$, where $\mu$ is a Borel probability measure on $\mathbf{R}^{d} \times \mathbf{R}^{d}$. Then

$$
E_{\hbar}\left(p, R_{\hbar}\right)^{2} \leq \operatorname{dist}_{\mathrm{MK}, 2}(p, \mu)^{2}+\frac{1}{2} d \hbar .
$$

(3) For each $R \in \mathcal{D}(\mathfrak{H})$, one has

$$
\operatorname{dist}_{\mathrm{MK}, 2}\left(p, \widetilde{W}_{\hbar}[R]\right)^{2} \leq E_{\hbar}(p, R)^{2}+\frac{1}{2} d \hbar \leq 2 E_{\hbar}(p, R)^{2} .
$$

Corollary 3.4. Let $R$ be a Töplitz operator of symbol $(2 \pi \hbar)^{d} \mu$. Then

$$
E_{\hbar}(\mu, R)^{2}=\frac{1}{2} d \hbar
$$

and an optimal coupling is

$$
Q_{0}(x, \xi):=\mu(x, \xi)|x, \xi\rangle\langle x, \xi| .
$$

Proof. the first equality follows from the items (1) and (2) in Theorem 3.3 and the second from the easy computation

$$
\int_{\mathbf{R}^{d} \times \mathbf{R}^{d}} \operatorname{trace}\left(c_{\hbar}(x, \xi) Q_{0}(x, \xi)\right) d x d \xi=\frac{1}{2} d \hbar .
$$

We will also need the following "triangle inequality" proven in Appendix A.

Theorem 3.5. Let $f, g$ be two probability densities on $\mathbf{R}^{2 d}$ and $R$ be a density operator on $L^{2}\left(\mathbf{R}^{d}\right)$ satisfying

$$
\int_{\mathbf{R}^{2 d}}\left(p^{2}+q^{2}\right)(f(p, q)+g(p, q)) d p d q<\infty \text { and } \operatorname{trace}\left(-\hbar^{2} \Delta+x^{2}\right) R<\infty .
$$

The following inequality holds true:

$$
E_{\hbar}\left(f, R_{1}\right) \leq \operatorname{dist}_{M K, 2}(f, g)+E_{\hbar}\left(g, R_{1}\right) .
$$


The proofs of Theorems 2.1, 2.2 and 2.3 will rely extensively on Theorem 2.7 in [10], a slight improvement of Theorem 2.7 in [12] in the special case $N=1$, that we recall now.

Theorem 3.6 (Theorem 2.7 in [10]). Let $\rho^{\text {in }}$ be a probability density on $\mathbf{R}^{2 d}$ satisfying

$$
\int_{\mathbf{R}^{2 d}}\left(p^{2}+q^{2}\right) \rho^{i n}(p, q) d p d q<\infty
$$

and let $R(t)$ and $\rho(t)$ the solutions of the von Neumann equation (7) and the Liouville equation (11) with initial conditions $R^{\text {in }}$ and $\rho^{\text {in }}$ respectively. then, for all $t \in \mathbf{R}$,

$$
E_{\hbar}(\rho(t), R(t))^{2} \leq E_{\hbar}\left(\rho^{i n}, R^{i n}\right)^{2} e^{\left(1+\max \left(1,4 \operatorname{Lip}(\nabla V)^{2}\right)\right)|t|} .
$$

Using the right inequality in item (3) of Theorem 3.3 we get the following corollary. Corollary 3.7. Let $R_{\hbar}(t)$ and $\rho(t)$ solve $(7)$ and $(11)$ respectively with initial data $R^{\text {in }}$ and $\widetilde{W}\left[R_{\hbar}\right]$ respectively. Let $R^{\text {in }}$ satisfies

$$
\int_{\mathbf{R}^{2 d}}\left(p^{2}+q^{2}\right) \widetilde{W}\left[R_{\hbar}\right](p, q) d p d q<\infty
$$

Then

$$
\operatorname{dist}_{\mathrm{MK}, 2}\left(\widetilde{W}\left[R_{\hbar}(t)\right], \rho(t)\right) \leq \sqrt{2} E_{\hbar}\left(\widetilde{W}\left[R_{\hbar}\right], R^{i n}\right) e^{\lambda|t|}
$$

\section{Proofs of Theorems 2.1, 2.2 And 2.3}

The conclusions of the Theorems 2.1, 2.2 and 2.3 are consequences of the following theorem which is the heart of this article and whose proof will occupy Section 5, 6 and 7 .

Theorem 4.1. Let $R^{\text {in }}$ satisfy the hypothesis of Theorems 2.1, 2.2 or 2.3. Then

(I) $E_{\hbar}\left(\widetilde{W}\left[R^{i n}\right], R^{i n}\right) \leq \frac{D_{I}}{\sqrt{2}} \max (\sqrt{\hbar}, \sqrt{\hbar} \mu(\hbar), \sqrt{\hbar \mu(\hbar) \nu(\hbar)}) \quad$ [case of Theorem 2.1]

(II) $E_{\hbar}\left(\widetilde{W}\left[R^{i n}\right], R^{i n}\right) \leq \frac{D_{I I}}{\sqrt{2}} \max \left(\sqrt{\hbar}, \sqrt{\hbar} \mu^{\prime}(\hbar), \sqrt{\mu^{\prime}(\hbar) \nu^{\prime}(\hbar)}\right) \quad$ [case of Theorem 2.2] (III) $E_{\hbar}\left(\widetilde{W}\left[R^{i n}\right], R^{i n}\right) \leq \frac{D_{I I I}}{\sqrt{2}} \max (\sqrt{\hbar}, \sqrt{\tau(\hbar)})$ [case of Theorem 2.3] Here $D_{I}, D_{I I}, D_{I I I}$ are given in (90), (50), (28) respectively.

Proof of Theorem 2.1. Item I and Corollary 3.7 give immediately the first result in Theorem 2.1. The second result is a corollary of the first thanks to Theorem B.8.

Proof of Theorem 2.2. Item II and Corollary 3.7 give again the first result in Theorem 4.1. The second result is a a corollary of the first thanks to Theorem B.8 and Proposition B.7.

Proof of Theorem 2.3. Finally, item III, Corollary 3.7 and Theorem B.8 gives the statement. 
Proof of the corollary of Theorem 2.1.

For $v \in \mathbf{R}$ let us define $\{v\}$ as the smallest integer greater or equal to $v$.

Lemma 4.2. For any $M, K, N>0$, there exist $C_{1}>0$ such that

$$
\|a\|_{M, K, N} \leq C_{1} \sup _{\substack{(x, \xi) \in \mathbf{R}^{2 d} \\\left|\beta_{1}\right|, \ldots,\left|\beta_{d}\right| \leq N+K}}\left(\xi^{2}+x^{2}+d\right)^{M+\left\{\frac{K}{2}\right\}}\left|\prod_{m=1}^{d} D_{(x, \xi)}^{\beta_{m}} a(x, \xi)\right|
$$

Proof. Let us first remark that, with the defintion (14),

$$
D_{z_{m}}^{\alpha_{m}} a_{k}(z)=\int D_{z_{m}}^{\alpha_{m}} a\left(e^{i t} z\right) P(t) e^{i k t} d t
$$

for a certain trigonometric polynomial $P$.

We have, for $K$ even,

$$
\begin{aligned}
\left(k_{l}^{2}+1\right)^{\frac{K}{2}} D_{z_{m}}^{\alpha_{m}} a_{k}(z) & =\int D_{z_{m}}^{\alpha_{m}} a\left(e^{i t} z\right) P(t)\left(\partial_{t}^{2}+1\right)^{\frac{K}{2}} e^{i k t} d t \\
& =\int e^{i k t}\left(\partial_{t}+i\right)^{\frac{K}{2}}\left(\partial_{t}-i\right)^{\frac{K}{2}}\left(D_{z_{m}}^{\alpha_{m}} a\left(e^{i t} z\right) P(t)\right) d t .
\end{aligned}
$$

Therefore, since $\left(\left|k_{l}\right|+1\right)^{K} \leq\left(k_{l}^{2}+1\right)^{\frac{K}{2}}$,

$$
\begin{aligned}
& \sup _{\left|\alpha_{1}\right|, \ldots,\left|\alpha_{d}\right| \leq N}\left(\left|k_{l}\right|+d\right)^{K}\left|\prod_{m=1}^{d} D_{z_{m}}^{\alpha_{m}} a_{k}(z)\right| \\
\leq & \sup _{\left|\alpha_{1}\right|, \ldots,\left|\alpha_{d}\right| \leq N}\left|\left(k_{l}^{2}+1\right)^{\frac{K}{2}} \prod_{m=1}^{d} D_{z_{m}}^{\alpha_{m}} a_{k}(z)\right| \\
\leq & C \prod_{n=1}^{d}\left(\left|z_{n}\right|^{2}+1\right)^{\frac{K}{2}} \sup _{\substack{\left|\alpha_{1}\right|, \ldots,\left|\alpha_{d}\right| \leq N+K \\
\leq}} \int\left|\prod_{m=1}^{d} D_{z_{m}}^{\alpha_{m}} a\left(e^{i t} z\right) e^{i k t}\right| d t \\
\leq & 2 \pi C\left(|z|^{2}+d\right)^{\frac{K}{2}} \sup _{\substack{\left|\alpha_{1}\right|, \ldots,\left|\alpha_{d}\right| \leq N+K \\
t \in[0,2 \pi]}}\left|\prod_{m=1}^{d} D_{z_{m}}^{\alpha_{m}} a\left(e^{i t} z\right)\right|
\end{aligned}
$$

Since the family of norms $\|\cdot\|_{M, K, N}$ is non decreasing in $K$, we conclude by noticing that $\|\cdot\|_{M, K, N} \leq\|\cdot\|_{M, 2\left\{\frac{K}{2}\right\}, N}$, plugging (24) in the definition (15) and define $C_{1}=$ $2 \pi C$.

Using Lemma 4.2, one sees easily that when $W_{\hbar}\left[\sqrt{R^{i n}}\right]$ satisfies the hypothesis of the Corollary of Theorem 2.1, the two hypothesis (1) and (2) of Theorem 2.1 are satisfied for $\mu(\hbar)=1$ and $\nu(\hbar)=\sqrt{\hbar}$ and the conclusion follows.

\section{Proof of Theorem 4.1 item (III)}

Let us start by a lemma, interesting per se. 
Lemma 5.1. Let $f$ be a probability density on $\mathbf{R}^{2 d}$ and $R$ be a density operator on $L^{2}\left(\mathbf{R}^{d}\right)$ satisfying

$$
\int_{\mathbf{R}^{2 d}}\left(p^{2}+q^{2}\right) f(p, q) d p d q<\infty \text { and } \operatorname{trace}\left(-\hbar^{2} \Delta+x^{2}\right) R<\infty .
$$

The following inequality holds true:

$$
E_{\hbar}(\widetilde{W}[R], R) \leq \sqrt{E_{\hbar}(f, R)^{2}+\frac{d \hbar}{2}}+E_{\hbar}(f, R) \leq(\sqrt{2}+1) E_{\hbar}(f, R) .
$$

Corollary 5.2. For any density operator satisfying trace $\left(-\hbar^{2} \Delta+x^{2}\right) R<\infty$,

$$
E_{\hbar}(\widetilde{W}[R], R) \leq(\sqrt{2}+1) \inf _{g \in \mathcal{P}\left(\mathbf{R}^{2 d}\right)} E_{\hbar}(g, R)
$$

Proof. By Theorem 3.3 (3),

$$
\operatorname{dist}_{\mathrm{MK}, 2}\left(\widetilde{W}[R, f)^{2} \leq E_{\hbar}(f, R)^{2}+\frac{d \hbar}{2}\right.
$$

and (25) follows by Theorem 3.5 and Theorem 3.3 (1).

Remark 5.3. The meaning of Corollary 5.2 is the following: thought $\widetilde{W}_{\hbar}[R]$ might not be the classical density "closets" to $R$, it belongs to a "ball" of radius $(\sqrt{2}+1)$ around $i t$.

Let us come back tp the proof of Theorem 4.1 item (III) and suppose satisfied Hypothesis 1 and 2 in Theorem 2.3 .

Let $F$ be given by Hypothesis 1 and let us define, for each $0<\epsilon \leq 1$

$$
F_{\epsilon}(x, \xi)=\left(\epsilon\left(x^{2}+\xi^{2}\right)+1\right)^{-1} F(x, \xi)
$$

so that $F_{\epsilon}$ has finite second moments.

Let $Q_{0}(x, \xi):=F_{\epsilon}(x, \xi)|x, \xi\rangle\langle x, \xi|$. By Corollary 3.4, $Q_{0}$ is a minimal coupling of $F_{\epsilon}$ and $\mathrm{OP}_{\hbar}^{T}\left((2 \pi \hbar)^{d} F_{\epsilon}\right)$.

Let us denote $A:=\sqrt{R^{i n}}$ and $T_{F_{\epsilon}}:=\mathrm{OP}_{\hbar}^{T}\left((2 \pi \hbar)^{d} F_{\epsilon}\right)$, and let

$$
Q(x, \xi)=A T_{F_{\epsilon}}^{-1 / 2} Q_{0}(x, \xi) T_{F_{\epsilon}}^{-1 / 2} A .
$$

By the hypothesis of boundedness of $A T_{F_{\epsilon}}^{-1 / 2}$ and $T_{F_{\epsilon}}^{-1 / 2} A, Q$ is a coupling between $R^{\text {in }}$ and $f_{A}(x, \xi):=F_{\epsilon}(x, \xi)\left\langle x, \xi\left|T_{F_{\epsilon}}^{-1 / 2} A^{2} T_{F_{\epsilon}}^{-1 / 2}\right| x, \xi\right\rangle$. Therefore, by Lemma 5.1,

$$
E_{\hbar}\left(\widetilde{W}\left[R_{\hbar}\right], R_{\hbar}\right) \leq \sqrt{E_{\hbar}\left(f_{A}, R_{\hbar}\right)^{2}+\frac{d \hbar}{2}}+E_{\hbar}\left(f_{A}, R_{\hbar}\right) .
$$

So the problem of estimating $E_{\hbar}\left(\widetilde{W}_{\hbar}\left[R^{i n}, R^{i n}\right)\right.$ reduces to estimating $E_{\hbar}\left(f_{A}, R_{\hbar}\right)$.

\section{Lemma 5.4.}

$$
c(x, \xi) Q_{0}(x, \xi)=\frac{d \hbar}{2} Q_{0}(x, \xi)
$$


Proof. Denoting by $T_{x, \xi}^{\hbar}, x, \xi \in \mathbf{R}^{d}$ the Weyl operators acting on any function $\psi \in$ $L^{2}\left(\mathbf{R}^{d}\right)$ by $T_{x, \xi}^{\hbar} \psi(y)=\psi(y-x) e^{i(\xi .(y-x / 2)}$ (see e.g. [13], Section 1), we have

$$
\begin{aligned}
c(x, \xi) Q_{0}(x, \xi) & =T(x, \xi) c(0,0) T(x, \xi)^{-1} Q_{0}(x, \xi) \\
& =T(x, \xi) c(0,0) Q_{0}(0,0) T(x, \xi)^{-1} \\
& =T(x, \xi) \frac{d \hbar}{2} Q_{0}(0,0) T(x, \xi)^{-1} \\
& =\frac{d \hbar}{2} Q_{0}(x, \xi) .
\end{aligned}
$$

Using Lemma 5.4, we get

$$
\begin{aligned}
E_{\hbar}\left(f_{A}, R\right)^{2} & \leq \int d x d \xi \operatorname{trace}(c(x, \xi) Q(x, \xi)) \\
& =\int d x d \xi \operatorname{trace}\left(c(x, \xi) A T_{F_{\epsilon}}^{-1 / 2} Q_{0}(x, \xi) T_{F_{\epsilon}}^{-1 / 2} A\right) \\
& =\int d x d \xi \operatorname{trace}\left(A T_{F_{\epsilon}}^{-1 / 2} c(x, \xi) Q_{0}(x, \xi) T_{F_{\epsilon}}^{-1 / 2} A\right) \\
& +\int d x d \xi \operatorname{trace}\left(\left[c(x, \xi), A T_{F_{\epsilon}}^{-1 / 2}\right] Q_{0}(x, \xi) T_{F_{\epsilon}}^{-1 / 2} A\right) \\
& =\frac{d \hbar}{2} \int d x d \xi \operatorname{trace} Q(x, \xi) \\
& +\int d x d \xi \operatorname{trace}\left(T_{F_{\epsilon}}^{-1 / 2} A\left[2 \xi \cdot y+2 x \cdot \nabla_{y}+y^{2}+\Delta_{y}, A T_{F_{\epsilon}}^{-1 / 2}\right] Q_{0}(x, \xi)\right) \\
& \leq \frac{d \hbar}{2} \\
& +\int d x d \xi\left\|T_{F_{\epsilon}}^{-1 / 2} A\left[2 \xi \cdot y+2 x . \nabla_{y}+y^{2}+\Delta_{y}, A T_{F_{\epsilon}}^{-1 / 2}\right]\right\| \operatorname{trace} Q_{0}(x, \xi) \\
& =\frac{d \hbar}{2}+\int d x d \xi\left\|T_{F_{\epsilon}}^{-1 / 2} A\left[2 \xi \cdot y+2 x \cdot \nabla_{y}+y^{2}+\Delta_{y}, A T_{F_{\epsilon}}^{-1 / 2}\right]\right\| F(x, \xi) \\
& \leq \frac{d \hbar}{2}+\int(1+|x|+|\xi|) F_{\epsilon}(d x, d \xi) \sum_{O \in \Omega}\left\|T_{F_{\epsilon}}^{-1 / 2} A\left[O, A T_{F_{\epsilon}}^{-1 / 2}\right]\right\| \\
& =\frac{d \hbar}{2}+\tau(\hbar) \int(1+|x|+|\xi|) F_{\epsilon}(d x, d \xi) .
\end{aligned}
$$

We conclude by using (27) and (26):

$$
E_{\hbar}\left(\widetilde{W}\left[R_{\hbar}\right], R_{\hbar}\right) \leq 2 \sqrt{\tau(\hbar) \int(1+|x|+|\xi|) F_{\epsilon}(d x, d \xi)+d \hbar} \leq 2 D_{I I I}(F) \max (\sqrt{\hbar}, \sqrt{\tau(\hbar)}) .
$$


with, since $F_{\epsilon} \leq F$,

$$
D_{I I I}(F)=2 \max \left(\sqrt{\int(1+|x|+|\xi|) F(d x, d \xi)}, \sqrt{d}\right) .
$$

\section{Proof of Theorem 4.1 item (II)}

We first notice the following elementary result.

Lemma 6.1. Let $\rho(\hbar), \tau^{\prime}(\hbar)$ be two functions such that $\rho(\hbar) \tau^{\prime}(\hbar)=o(1)$ as $\hbar \rightarrow 0$, and let $\sqrt{R^{i n}}$ satisfy the two following hypothesis

Then (1) and (2) in Theorem 2.3 hold true with

$$
\begin{gathered}
\left\|T_{F}^{-1 / 2} \sqrt{R^{i n}}\right\|,\left\|\sqrt{R^{i n}} T_{F}^{-1 / 2}\right\| \leq \rho(\hbar) \\
\sup _{O \in \Omega} \|\left[O, \sqrt{R^{i n}} T_{F}^{-1 / 2} \| \leq \tau^{\prime}(\hbar) .\right.
\end{gathered}
$$

$$
\tau(\hbar)=\rho(\hbar) \tau^{\prime}(\hbar)
$$

By Lemma 6.1, Theorem 4.1 item (II) is a consequence of the following result.

Proposition 6.2. Let us suppose that $R_{\hbar}$ satisfies the hypothesis of Theorem 2.2 for some $\mu^{\prime}, \nu^{\prime}, \epsilon$. Then $R_{\hbar}$ satisfies (1') and (2') for any function $F$ of the form $F\left(z_{1}, \ldots, z_{d}\right)=f\left(x_{1}^{2}+\xi_{1}^{2}\right) \ldots f\left(x_{d}^{2}+\xi_{d}^{2}\right)$ with $f$ satisfying $(33)$ below and $\rho$ and $\tau^{\prime}$ are given respectively by (42) and (48).

Proof. The proof of Proposition 6.2 is split in three steps. In Section 6.1 we will first realize $T_{F}^{-\frac{1}{2}}$, for $F$ satisfying (33) below, as a diagonal operator on the Hermite basis. This will simplify the computation of the matrix elements and therefore the norms of the operators arising in the hypothesis (1)' and (2)' in Lemma 6.1 out of the estimates of the matrix elements provided by hypothesis (1) and (2) in Theorem 2.2.This is done in Sections 6.2 and 6.3 respectively.

6.1. Construction of $T_{F}^{-\frac{1}{2}}$. Let us take $F$ in the form $F=f\left(x_{1}^{2}+\xi_{1}^{2}\right) \ldots f\left(x_{d}^{2}+\xi_{d}^{2}\right)$. In other words, calling $h_{0}(x, \xi)=\left(\xi^{2}+x^{2}\right) / 2$ defined on $T^{*}(\mathbf{R}), F=f\left(h_{0}\right)^{\otimes d}$. It is easy to see that, since $|x, \xi\rangle\langle x, \xi|$ evolves by conjugation by the quantum flow of the harmonic oscillator by the action of the classical underlying flow on $(x, \xi), \mathrm{Op}_{\hbar}^{T}\left((2 \pi \hbar)^{d} F\right)$ is invariant by conjugation by the quantum flow of one dimensional harmonic oscillators $\mathcal{H}_{0}$ as soon as $F$ is invariant by the action of the corresponding classical flow. 
Therefore $\mathrm{Op}_{\hbar}^{T}\left((2 \pi \hbar)^{d} F\right)=G\left(\mathcal{H}_{0}\right)^{\otimes d}$ for some $G$ and, by (16) and after denoting $I=(1, \ldots, 1) \in \mathbf{N}^{d}$,

$\mathrm{OP}_{\hbar}^{T}\left((2 \pi \hbar)^{d} F\right)=(2 \pi \hbar)^{d} \sum_{j \in \mathbf{N}^{d}} G^{\otimes d}\left(\left(j+\frac{1}{2} I\right) \hbar\right)\left|H_{j}\right\rangle\left\langle H_{j}\right|=\left(\sum_{j \in \mathbf{N}}(2 \pi \hbar) G\left(\left(j+\frac{1}{2}\right) \hbar\right)\left|h_{j}\right\rangle\left\langle h_{j}\right|\right)^{\otimes d}$.

When there is no confusion we will drop the notation $I$ and write for any $j \in \mathbf{N}^{d}$,

$$
j+\frac{1}{2} I=j+\frac{1}{2} .
$$

The function $G$ can be evaluated on the spectrum of $\mathcal{H}_{0}$ by the following argument: let $z^{\prime} \in \mathbf{C}, j \in \mathbf{N}$ and $\left|z^{\prime}, \hbar\right\rangle$ be defined by (17). By a simple computation using the generating function of the Hermite polynomials (see Section 7.1) we easily find that

$$
\left\langle z^{\prime} \mid h_{j}\right\rangle=\frac{z^{\prime j}}{\sqrt{j ! \hbar^{j}}} e^{-\frac{\left|z^{\prime}\right|^{2}}{2 \hbar}}
$$

so that

$$
\left\langle z^{\prime} \mid z\right\rangle=\sum_{j \in \mathbf{N}}\left\langle z^{\prime} \mid h_{j}\right\rangle\left\langle h_{j} \mid z\right\rangle=e^{\frac{z^{\prime} \bar{z}}{\hbar}} e^{-\frac{|z|^{2}}{2 \hbar}} e^{-\frac{\left|z^{\prime}\right|^{2}}{2 \hbar}}
$$

Then

$$
\begin{aligned}
G((j+1 / 2) \hbar)\left\langle z^{\prime} \mid h_{j}\right\rangle & =\left\langle z^{\prime}\left|G\left(\mathcal{H}_{0}\right)\right| h_{j}\right\rangle=\left\langle z^{\prime}\left|\mathrm{OP}_{\hbar}^{T}\left(f\left(h_{0}\right)\right)\right| h_{j}\right\rangle \\
(\text { by }(29)) & =\int\left\langle z^{\prime}|f(z \bar{z})| z\right\rangle \frac{\bar{z}^{j}}{\sqrt{j ! \hbar^{j}}} e^{-\frac{|z|^{2}}{2 \hbar}} d z d \bar{z} /(2 \pi \hbar) \\
& =\int f(z \bar{z})\left\langle z^{\prime} \mid z\right\rangle \frac{\bar{z}^{j}}{\sqrt{j ! \hbar^{j}}} e^{-\frac{|z|^{2}}{2 \hbar}} d z d \bar{z} /(2 \pi \hbar) \\
(\text { by (30)) } & =\frac{1}{\hbar} \frac{z^{\prime j}}{\sqrt{j ! \hbar^{j}}} e^{-\frac{\left|z^{\prime}\right|^{2}}{2 \hbar}} \int_{0}^{\infty} f\left(\rho^{2}\right) \frac{\rho^{2 j}}{j ! \hbar^{j}} e^{-\rho^{2} / \hbar} \rho d \rho \\
\text { (by (29) again) } & =\left\langle z^{\prime} \mid h_{j}\right\rangle \frac{1}{\hbar} \int_{0}^{\infty} f\left(\rho^{2}\right) \frac{\rho^{2 j}}{j ! \hbar^{j}} e^{-\rho^{2} / \hbar} \rho d \rho
\end{aligned}
$$

so that

$$
G((j+1 / 2) \hbar)=\int_{\mathbf{R}^{+}} f\left(\rho^{2}\right) \frac{\left(\rho^{2} / \hbar\right)^{j}}{j ! \hbar} e^{-\rho^{2} / \hbar} \rho d \rho,
$$

In particular, since $0 \neq f \geq 0, G((j+1 / 2) \hbar)>0$. Therefore $\mathrm{OP}_{\hbar}^{T}(F)^{-1 / 2}$ can be defined as an unbounded operator by

$$
\mathrm{OP}_{\hbar}^{T}\left((2 \pi \hbar)^{d} F\right)^{-1 / 2}\left|h_{j}\right\rangle=\left(((2 \pi \hbar) G)^{-1 / 2}\right)^{\otimes d}((j+1 / 2) \hbar)\left|h_{j}\right\rangle, \forall j \in \mathbf{N}^{d} .
$$

Remark 6.3. Note that, when $f \in C^{2}(\mathbf{R})$, the stationary phase lemma gives that $G(A) \sim f(A)$ as $j \rightarrow \infty, \hbar \rightarrow 0,(j+1 / 2) \hbar \rightarrow A<\infty$. Moreover, (31) can be inverted 
by

$$
\hbar \sum_{j \in \mathbf{N}} G((j+1 / 2) \hbar)(1-\lambda)^{j}=\mathbb{L}(f)(\lambda),
$$

where $\mathbb{L}$ is the Laplace transform.

Note also that $G$ is bounded since $\left(\rho^{2} / \hbar\right)^{j} e^{-\rho^{2} / \hbar} \leq j^{j} e^{-j} \leq j$ ! and $f$ is integrable, in fact $G \leq 1 / \hbar$.

Note finally that the Gaussian case is explicitly computable.

Lemma 6.4. Let us suppose there exist $\nu>\frac{3}{2}$, and $C_{1}, C_{0}>0$ such that,

$$
\left\{\begin{array}{c}
-2 C_{0}\left(\rho^{2}+1\right)^{-\nu-1} \leq f^{\prime}\left(\rho^{2}\right) \leq 0 \\
C_{1}\left(\rho^{2}+1\right)^{-\nu} \leq f\left(\rho^{2}\right) .
\end{array}\right.
$$

Then, for all $A \in \mathbf{R}^{+}$

$$
C^{\prime}(A+1)^{-\nu} \leq G(A)
$$

where $C^{\prime}$ is given by (36) below.

Moreover

$$
\begin{aligned}
G((j+1 / 2) \hbar)-G((j+3 / 2) \hbar) & =-\hbar \int_{0}^{\infty} f^{\prime}\left(\rho^{2}\right) \frac{\left(\rho^{2} / \hbar\right)^{j+1}}{(j+1) ! \hbar} e^{-\rho^{2} / \hbar} \rho d \rho \\
& \left.\leq 4^{\nu+1} \hbar C_{0}(j \hbar+1 / 2)\right)^{-(\nu+1)}
\end{aligned}
$$

Note that the condition on $\nu$ is compatible with the finiteness of $\int(|x|+|\xi|) F(d x, d \xi)$. Proof. We first remark that $C_{1}\left(\rho^{2}+1\right)^{-\nu}$ implies that, for all $\alpha>0$ and $C=C_{1}\left(\frac{1+\alpha}{\alpha}\right)^{\nu}$.

$$
\begin{aligned}
C \rho^{-2 \nu} & \leq f\left(\rho^{2}\right), & & \rho^{2} \geq \alpha \\
C_{1} & \leq f\left(\rho^{2}\right) . & & \rho^{2}<\alpha
\end{aligned}
$$

Since $f>0$ we have, for $j \geq \nu$, by (33)

$$
\begin{aligned}
G\left(\left(j+\frac{1}{2}\right) \hbar\right) & \geq \int_{\alpha}^{\infty} f\left(\rho^{2}\right) \frac{\left(\rho^{2} / \hbar\right)^{j}}{j ! \hbar} e^{-\rho^{2} / \hbar} d\left(\rho^{2} / 2\right) \\
& \geq \int_{\alpha}^{\infty} \frac{C}{\hbar^{\nu} j(j-1) \ldots(j-\nu+1)} \frac{\left(\rho^{2} / \hbar\right)^{j-\nu}}{(j-\nu) ! \hbar} e^{-\rho^{2} / \hbar} d\left(\rho^{2} / 2\right) \\
& \geq C(j \hbar)^{-\nu}\left(1-\int_{0}^{\frac{\alpha}{\hbar}} \frac{x^{j-\nu} e^{-x}}{(j-\nu) !} d x\right) \\
j^{\prime}=j-\nu & \geq C(j \hbar)^{-\nu}\left(1-(\alpha / \hbar)^{j^{\prime}}\left(j^{\prime}\right)^{-j^{\prime}} e^{j^{\prime}} \int_{0}^{\infty} e^{-x} d x\right) \quad \text { (by Stirling) } \\
& \geq C(j \hbar)^{-\nu}\left(1-e^{j^{\prime}\left(1+\log \frac{\alpha}{j^{\prime} \hbar}\right)}\right) \\
& \geq C(j \hbar)^{-\nu}\left(1-\frac{1}{e}\right) \text { for } j \hbar \geq e^{2} \alpha+\nu \hbar . \\
& \geq C \frac{e-1}{e}\left(\left(j+\frac{1}{2}\right) \hbar+1\right)^{-\nu}
\end{aligned}
$$


For $j=0$ we have that

$$
G(\hbar / 2)=\frac{1}{2} \int_{\mathbf{R}^{+}} f(\lambda) e^{-\frac{\lambda}{\hbar}} \frac{d \lambda}{\hbar}=\frac{1}{2} \int_{0}^{1} f(\hbar \lambda) e^{-\lambda} d \lambda \geq \frac{C_{1}}{2 e}:=C^{\prime \prime}
$$

For $0<A:=j \hbar \leq e^{2} \alpha+\nu \hbar$, we get, since $j ! \leq K \sqrt{2 \pi} j^{j} \sqrt{j} e^{-j}$ for some $K>1$,

$$
\begin{aligned}
G((j+1 / 2) \hbar)=G(A+\hbar / 2) & \geq \frac{e^{\frac{A}{\hbar}}}{2 K \sqrt{2 \pi} \sqrt{A}} \int_{\mathbf{R}^{+}} f(\lambda) e^{-\frac{\lambda-A \log \left(\frac{\lambda}{A}\right)}{\hbar}} \frac{d \lambda}{\sqrt{\hbar}} \\
& \geq \frac{e^{\frac{A}{\hbar}}}{2 K \sqrt{2 \pi} \sqrt{A}} \int_{A-\frac{1}{2} \sqrt{\hbar A}}^{A+\frac{1}{2} \sqrt{\hbar A}} f(\lambda) e^{-\frac{\lambda-A \log \left(\frac{\lambda}{A}\right)}{\hbar}} \frac{d \lambda}{\sqrt{\hbar}} \\
\text { (since } \left.\log (1+x)-x \geq-\frac{1}{2} x^{2}\right) & \geq \frac{1}{2 K \sqrt{2 \pi}} \int_{-\frac{1}{2}}^{+\frac{1}{2}} f(A+\sqrt{\hbar A} \mu) e^{-\frac{\mu^{2}}{2}} d \mu \\
& \geq \frac{1}{2 K \sqrt{2 \pi}}-\inf _{\frac{1}{2} \leq \mu \leq+\frac{1}{2}} f(A+\sqrt{\hbar A} \mu) \int_{-\frac{1}{2}}^{+\frac{1}{2}} e^{-\frac{\mu^{2}}{2}} d \mu \\
& \geq \frac{C_{1} e^{-\frac{1}{8}}}{K \sqrt{2 \pi}}:=C^{\prime \prime \prime}>0
\end{aligned}
$$

Therefore (34) holds true if we define

$$
C^{\prime}=\inf \left(C, C^{\prime \prime}, C^{\prime \prime \prime}\right)=C_{1} / 2 \pi e^{\frac{1}{8}}
$$

Moreover, by integration by part,

$$
\begin{aligned}
G((j+1 / 2) \hbar) & =-\int_{0}^{\infty} \frac{\left(\rho^{2} / \hbar\right)^{j+1}}{(j+1) ! \hbar} \frac{d}{d\left(\frac{\rho^{2}}{\hbar}\right)}\left(f\left(\rho^{2}\right) e^{-\rho^{2} / \hbar}\right) \rho d \rho \\
& =\int_{0}^{\infty} f\left(\rho^{2}\right) \frac{\left(\rho^{2} / \hbar\right)^{j+1}}{(j+1) ! \hbar} e^{-\rho^{2} / \hbar} \rho d \rho \\
& -\int_{0}^{\infty} \hbar f^{\prime}\left(\rho^{2}\right) \frac{\left(\rho^{2} / \hbar\right)^{j+1}}{(j+1) ! \hbar} e^{-\rho^{2} / \hbar} \rho d \rho
\end{aligned}
$$

This proves the first part of (35).

For $0<\eta<\infty$ we decompose the middle term in (35) in two parts. Since by the inequality of $(33),-f^{\prime}\left(\rho^{2}\right) \leq 2 C_{0}$, we first compute

$$
-\int_{0}^{\sqrt{\eta}} \hbar f^{\prime}\left(\rho^{2}\right) \frac{\left(\rho^{2} / \hbar\right)^{j+1}}{(j+1) ! \hbar} e^{-\rho^{2} / \hbar} \rho d \rho \leq \hbar C_{0} \int_{0}^{\eta / \hbar} \frac{(\lambda)^{j+1}}{(j+1) !} e^{-\lambda} d \lambda \leq \hbar C_{0}
$$


Using the first inequality of (33) we get that

$$
\begin{aligned}
-\int_{\sqrt{\eta}}^{\infty} \hbar f^{\prime}\left(\rho^{2}\right) \frac{\left(\rho^{2} / \hbar\right)^{j+1}}{(j+1) ! \hbar} e^{-\rho^{2} / \hbar} \rho d \rho & =-\frac{1}{2} \int_{\eta}^{\infty} f^{\prime}\left(\lambda^{\prime}\right) \frac{e^{-\frac{\lambda^{\prime}-(j+1) \hbar \log \lambda^{\prime}}{\hbar}}}{\hbar^{j+1}(j+1) !} d \lambda^{\prime} \\
& =-\frac{\hbar}{2} \int_{\eta / \hbar}^{\infty} f^{\prime}(\hbar \lambda) \frac{\lambda^{j+1}}{(j+1) !} e^{-\lambda} d \lambda \\
& \leq \hbar C_{0} \int_{0}^{\infty}(\lambda \hbar+1)^{-(\nu+1)} \frac{\lambda^{j+1}}{(j+1) !} e^{-\lambda} d \lambda \\
& \leq \hbar C_{0} \int_{0}^{\infty} \min \left(\frac{\hbar^{-(\nu+1)}}{(j+1) j(j-1)} \frac{\lambda^{j-\nu}}{(j-2) !} \frac{\lambda^{j+1}}{(j+1) !}\right) e^{-\lambda} d \lambda \\
& \leq \hbar C_{0} \min \left((\hbar(j-(\nu-1)))^{-(\nu+1)}, 1\right) \\
& \leq \hbar C_{0} 2^{\nu+1}(\hbar(j-(\nu-1))+1)^{-(\nu+1)} \\
& \leq 4^{\nu+1} \hbar C_{0}\left(j \hbar+\frac{1}{2}\right)^{-(\nu+1)} .
\end{aligned}
$$

Let us summarize what has been done in this section: out of any $F=f^{\otimes d}$ where $f$ satisfies condition (33), we have defined $\mathrm{OP}_{\hbar}^{T}\left((2 \pi \hbar)^{d} F\right)^{-1 / 2}$ by the formula

$$
\mathrm{OP}_{\hbar}^{T}\left((2 \pi \hbar)^{d} F\right)^{-1 / 2}=(2 \pi \hbar)^{-d / 2} \sum_{j \in \mathbf{N}^{d}} g^{\otimes d}((j+1 / 2) \hbar)\left|H_{j}\right\rangle\left\langle H_{j}\right|,
$$

in the sense that

$$
\left\langle H_{j}\left|\mathrm{Op}_{\hbar}^{T}\left((2 \pi \hbar)^{d} F\right)^{-1 / 2}\right| H_{i}\right\rangle=(2 \pi \hbar)^{-d / 2} \delta_{i, j} g^{\otimes d}((j+1 / 2) \hbar)
$$

where $g$ satisfies, for all $A \in \mathbf{R}^{+}$,

$$
\begin{aligned}
g(A) & \leq\left(C^{\prime}\right)^{-\frac{1}{2}}(A+1)^{\frac{\nu}{2}} \\
g^{-2}\left(\left(j+\frac{1}{2}\right) \hbar\right)-g^{-2}\left(\left(j+\frac{3}{2}\right) \hbar\right) & \leq 4^{\nu+1} \hbar C_{0}\left(A+\frac{1}{2}\right)^{-(\nu+1)}
\end{aligned}
$$

for $C, C_{0}$ defined in (33) and $C^{\prime}$ in (36).

From now on, we will suppose that $f$ satisfies (33) for some $\nu>\frac{3}{2}$.

\subsection{Hypothesis (1) in Theorem $2.2 \Longrightarrow\left(1^{\prime}\right)$ in Lemma 6.1.}

Condition (1') is easily checkable by the control of the decay of $\left\langle H_{j+k}\left|\sqrt{R^{i n}}\right| H_{j}\right\rangle$ as both $j$ and $|k| \rightarrow \infty$.

Let us recall that, [5] Chapter VI, Paragraph 9, Exercise 54, the operator norm of any operator $B$ on a separable Hilbert space $\mathcal{H}$, satisfies, for any orthonormal basis $\{|j\rangle\}$ of $\mathcal{H}$

$$
\|B\| \leq \max \left(\sup _{j} \sum_{j^{\prime}}\left|\left\langle j^{\prime}|B| j\right\rangle\right|, \sup _{j^{\prime}} \sum_{j}\left|\left\langle j^{\prime}|B| j\right\rangle\right|\right)
$$

In our case $B=\sqrt{R^{i n}} \mathrm{OP}_{\hbar}^{T}\left((2 \pi \hbar)^{d} F\right)^{-1 / 2}$ so that, after taking $\nu=\frac{3}{2}+2 \epsilon, 0<\epsilon \leq 1$, (say) in (37),

$$
\langle j+k|B| j\rangle=(2 \pi \hbar)^{-d / 2}\left\langle j+k\left|\sqrt{R^{i n}}\right| j\right\rangle g^{\otimes d}((j+1 / 2) \hbar)
$$


with $\sqrt{R^{i n}}$ self-adjoint and $g$ satisfying (38).

Therefore, one has, under the condition (1) in Theorem 2.2,

$$
\left\langle j^{\prime}\left|\sqrt{R^{i n}} \mathrm{OP}_{\hbar}^{T}\left((2 \pi \hbar)^{d} F\right)^{-1 / 2}\right| j\right\rangle \leq\left(C^{\prime}\right)^{-\frac{d}{2}} \mu^{\prime}(\hbar) \prod_{1 \leq l \leq d}\left(\left|j_{l}-j_{l}^{\prime}\right|+1\right)^{-\frac{3}{2}-\epsilon}
$$

Since

$$
\sum_{j_{l}^{\prime}=0}^{\infty}\left(\left|j_{l}-j_{l}^{\prime}\right|+1\right)^{-\frac{3}{2}-\epsilon}=\sum_{j_{l}=0}^{\infty}\left(\left|j_{l}-j_{l}^{\prime}\right|+1\right)^{-\frac{3}{2}-\epsilon} \leq 1,
$$

and $C^{\prime}=C_{1} /\left(2 \pi e^{\frac{1}{8}}\right),(40)$ gives

$$
\left\|\sqrt{R^{i n}} \mathrm{OP}_{\hbar}^{T}(F)^{-1 / 2}\right\| \leq(2 \pi)^{\frac{d}{2}} e^{\frac{d}{16}} C_{1}^{-\frac{d}{2}} \mu^{\prime}(\hbar)
$$

Therefore, the tightness condition (1') in Proposition 6.2 is satisfied with

$$
\rho(\hbar)=(2 \pi)^{\frac{d}{2}} e^{\frac{d}{16}} C_{1}^{-\frac{d}{2}} \mu^{\prime}(\hbar) .
$$

\subsection{Hypothesis (1) and (2) in Theorem $2.2 \Longrightarrow\left(2^{\prime}\right)$ in Lemma 6.1.}

In order to prove the semiclassical condition $\left(2^{\prime}\right)$, we first remark that

$$
\left[O, \sqrt{R^{i n}} T_{F}^{-1 / 2}\right]=\sqrt{R^{i n}}\left[O, T_{F}^{-1 / 2}\right]+\left[O, \sqrt{R^{i n}}\right] T_{F}^{-1 / 2} .
$$

The second term of the right hand side is treated exactly the same way as for $\left(1^{\prime}\right)$ :

- for $O=\mathcal{H}_{l}=-\hbar^{2} \nabla_{l}^{2}+x_{l}^{2}$ we first note that

$$
\left\langle j^{\prime}\left|\left[\mathcal{H}_{l}, \sqrt{R^{i n}}\right]\right| j\right\rangle=\left(j_{l}^{\prime}-j_{l}\right) \hbar\left\langle j^{\prime}\left|\sqrt{R^{i n}}\right| j\right\rangle,
$$

so that, by (1) in Theorem 2.2 ,

$$
\left|\left\langle j^{\prime}\left|\left[\mathcal{H}_{l}, \sqrt{R^{i n}}\right] T_{F}^{-\frac{1}{2}}\right| j\right\rangle\right| \leq C^{\prime-d} \hbar \mu^{\prime}(\hbar) \prod_{1 \leq l \leq d}\left(\left|j_{l}-j_{l}^{\prime}\right|+1\right)^{-1-\epsilon} .
$$

Since $\prod_{1 \leq l \leq d}\left(\left|j_{l}-j_{l}^{\prime}\right|+1\right)^{-1-\epsilon} \leq 2 / \epsilon$ and $C^{\prime}=C_{1} /\left(21 e^{\frac{1}{8}}\right)$, we get, by $(40)$,

$$
\left\|\left[\mathcal{H}_{l}, \sqrt{R^{i n}}\right] T_{F}^{-\frac{1}{2}}\right\| \leq(2 \pi)^{\frac{d}{2}} e^{\frac{d}{16}}\left(C_{1} \epsilon^{2}\right)^{-\frac{d}{2}} \hbar \mu^{\prime}(\hbar) .
$$

- for $O \neq \mathcal{H}_{l},(2)$ in Theorem 2.2 gives precisely by the same argument

$$
\left\|\left[O, \sqrt{R^{i n}}\right] T_{F}^{-\frac{1}{2}}\right\| \leq(2 \pi)^{\frac{d}{2}} e^{\frac{d}{16}}\left(C_{1} \epsilon^{2}\right)^{-\frac{d}{2}} \nu^{\prime}(\hbar) .
$$

For the first term in the right hand-side of (43), let us start with $O_{l}=x_{l}+\hbar \partial_{x_{l}}, l=$ $1, \ldots, d$, and let us remember that $O_{l} H_{j}=\sqrt{\left(j_{l}+1\right) \hbar} H_{j+1_{l}}$ and $O_{l}^{*} H_{j}=\sqrt{j_{l} \hbar} H_{j-1_{l}}$, as is easily checked. 
Defining $1_{l} \in \mathbf{N}^{d}$ by $\left(1_{l}\right)_{k}=\delta_{k, l}, k, l=1, \ldots, d$, one has, with $A:=\sqrt{R^{i n}}$,

$$
\begin{aligned}
& \left\langle H_{i}\left|A\left[O_{l}, T^{-1 / 2}\right]\right| H_{j}\right\rangle \\
= & \left(g^{\otimes d}\left(\left(j+\frac{1}{2}\right) \hbar\right)-g^{\otimes d}\left(\left(j+1_{l}+\frac{1}{2}\right) \hbar\right)\right)\left(\sqrt{\left(j_{l}+1\right) \hbar}\left\langle H_{i}|A| H_{j+1_{l}}\right\rangle\right.
\end{aligned}
$$

so that (remember $g=G^{-\frac{1}{2}}$ )

$$
\begin{aligned}
& \left|\left\langle H_{j+k}\left|A\left[O_{l}, T^{-1 / 2}\right]\right| H_{j}\right\rangle\right| \leq \\
& \left.\left|G\left(\left(j_{l}+\frac{1}{2}\right) \hbar\right)^{-1 / 2}-G\left(\left(j_{l}+\frac{3}{2}\right) \hbar\right)^{-1 / 2}\right|\right) \sqrt{\left(j_{l}+1\right) \hbar} \mid\left(\left(j_{l}+\frac{1}{2}\right) \hbar+1\right)^{-\frac{1}{2}-\epsilon}\left(\left|k_{l}-1\right|+1\right)^{-2-\epsilon} \\
& \left.\times \mu^{\prime}(\hbar) \prod_{m \neq l} \mid g\left(j_{m}+\frac{1}{2}\right) \hbar\right)\left|\left(\left(j_{m}+\frac{1}{2}\right) \hbar+1\right)\right|^{-\frac{1}{2}-\epsilon}\left(\left|k_{m}\right|+1\right)^{-2-\epsilon} .
\end{aligned}
$$

- the second factor in the right hand-side of (46), namely

$$
\left.C \prod_{m \neq l} \mid G\left(j_{m}+\frac{1}{2}\right) \hbar\right)\left|\left(\left(j_{m}+\frac{1}{2}\right) \hbar+1\right)\right|^{-\frac{1}{2}-\epsilon}\left(\left|k_{m}\right|+1\right)^{-1-\epsilon}
$$

will gives again, aa for the derivation of (41) by Lemma 40, a contribution to $\left\|A\left[O, T^{-1 / 2}\right]\right\|$ bounded by $\mu^{\prime}(\hbar)(2 \pi)^{d-1} e^{\frac{d-1}{8}} C_{1}^{-(d-1)}$.

- for the estimate of the first factor in the right hand-side of (46),

$$
\begin{aligned}
& \left.\left|G\left(\left(j_{l}+\frac{1}{2}\right) \hbar\right)^{-1 / 2}-G\left(\left(j_{l}+\frac{3}{2}\right) \hbar\right)^{-1 / 2}\right|\right) \sqrt{\left(j_{l}+1\right) \hbar} \mid\left(\left(j_{l}+\frac{1}{2}\right) \hbar+1\right)^{-\frac{1}{2}-\epsilon}\left(\left|k_{l}-1\right|+1\right)^{-2-\epsilon} \\
\leq & \left.\left|G\left(\left(j_{l}+\frac{1}{2}\right) \hbar\right)^{-1 / 2}-G\left(\left(j_{l}+\frac{3}{2}\right) \hbar\right)^{-1 / 2}\right|\right)\left(\left(j_{l}+\frac{1}{2}\right) \hbar+1\right)^{-\epsilon}\left(\left|k_{l}-1\right|+1\right)^{-2-\epsilon}
\end{aligned}
$$

we first remark that, since $f^{\prime} \leq 0$ and (35) holds true, one can extend $G$ to $\mathbf{R}^{+}$with $G^{\prime}(x) \leq 0, x \in \mathbf{R}^{+}$. Therefore,

$$
\begin{aligned}
& \left|G\left(\left(j_{l}+\frac{1}{2}\right) \hbar\right)^{-1 / 2}-G\left(\left(j_{l}+\frac{3}{2}\right) \hbar\right)^{-1 / 2}\right| \\
= & \frac{1}{2}\left|\int_{)}^{\hbar} G^{\prime}\left(\left(j+\frac{1}{2}\right) \hbar+\lambda\right) G\left(\left(j+\frac{1}{2}\right) \hbar+\lambda\right)^{-\frac{3}{2}} d \lambda\right|
\end{aligned}
$$

$\left(\right.$ since $\left.G^{\prime} \leq 0 \leq G\right) \leq \frac{1}{2} \sup _{0 \leq \lambda \leq \hbar}\left(G\left(\left(j+\frac{1}{2}\right) \hbar+\lambda\right)^{-\frac{3}{2}}\right) \mid G\left(\left(j+\frac{3}{2}\right) \hbar-G\left(\left(j+\frac{1}{2}\right) \hbar\right) \mid\right.$

$$
=\frac{1}{2} \sup _{0 \leq \lambda \leq \hbar}\left(g\left(\left(j+\frac{1}{2}\right) \hbar+\lambda\right)^{3}\right) \mid G\left(\left(j+\frac{3}{2}\right) \hbar-G\left(\left(j+\frac{1}{2}\right) \hbar\right) \mid\right.
$$

(by $(34)$ and $(35)) \leq 4^{\nu+1} \hbar C_{0}\left(2 \pi e^{\frac{1}{8}} C_{1}\right)^{-\frac{3}{2}}(j \hbar+1 / 2)^{\frac{\nu}{2}-1}$.

Recalling that $\nu=\frac{3}{2}+2 \epsilon$ we get that the first factor in the right hand-side of (43) is bounded by

$$
4^{\nu+1} \hbar C_{0}\left(\left|k_{l}-1\right|+1\right)^{-1-\epsilon}=4^{\nu+1} \hbar C_{0}\left(2 \pi e^{\frac{1}{8}} C_{1}\right)^{-\frac{3}{2}}\left(\left|(j+k)_{l}-j_{l}-1\right|+1\right)^{-1-\epsilon}
$$

Therefore, it gives a contribution to $\left\|A\left[O, T^{-1 / 2}\right]\right\|$, by (40) again, bounded by $8 \hbar C_{0}$.

Putting together the two contributions of (46), we get that

$$
\left\|A\left[O, T^{-1 / 2}\right]\right\| \leq 4^{\nu+1} C_{0}\left(2 \pi e^{\frac{1}{8}} C_{1}\right)^{-\frac{d}{2}-1} \hbar \mu^{\prime}(\hbar) .
$$

The term with $O_{l}^{*}=x_{l}-\partial_{x_{l}}=\left(x_{l}+\partial_{x_{l}}\right)$ is done the same way. 
Putting together (44), (45) and (47), we get the semiclassical condition (2') in Proposition 6.2 with

$$
\tau^{\prime}(\hbar)=\left(2 \pi e^{\frac{1}{8}} C_{1}\right)^{-\frac{d}{2}-1}\left(4^{\nu+1} C_{0}+2 \pi e^{\frac{1}{8}} C_{1} \epsilon^{-d}\right) \sup \left(\hbar \mu^{\prime}(\hbar), \nu^{\prime}(\hbar)\right) .
$$

so that, by (42), Theorem 4.1 item (II) is a consequence of item (III) with

$$
\tau(\hbar)=\rho(\hbar) \tau^{\prime}(\hbar)=\left(2 \pi e^{\frac{1}{8}} C_{1}\right)^{-d-1}\left(4^{\nu+1} C_{0}+2 \pi e^{\frac{1}{8}} C_{1} \epsilon^{-d}\right) \sup \left(\hbar \mu^{\prime}(\hbar)^{2}, \nu^{\prime}(\hbar) \mu^{\prime}(\hbar)\right)
$$

Taking $f\left(\rho^{2}\right)=C_{1}\left(1+\rho^{2}\right)^{-2-2 \epsilon}$ with $2 \pi e^{\frac{1}{8}} C_{1} \leq 1$ leads to

$$
\tau(\hbar) \leq\left(4^{6}(2+\epsilon)+\epsilon^{-d}\right) \sup \left(\hbar \mu^{\prime}(\hbar)^{2}, \nu^{\prime}(\hbar) \mu^{\prime}(\hbar)\right)
$$

Therefore item $I I)$ is proven for

$$
D_{I I}=\left(4^{6}(2+\epsilon)+\epsilon^{-d}\right) D_{I I I}
$$

\section{Proof of Theorem 4.1 item (I)}

In order to prove Theorem 4.1 item (I) as a corollary of item (II), we need to estimate, under the hypothesis (1) and (2) of Theorem 2.1, the matrix elements of $\sqrt{R^{i n}}$ and $\left[O, \sqrt{R^{i n}}\right], O \in \Omega_{1}$, between semiclassical Hermite functions, uniformly in $\hbar$. We will carry out this computation out of their Wigner transform, or equivalently their Weyl symbols (which we recall to be their Wigner functions multiplied by $(2 \pi \hbar)^{d}$ ).

Of course no exact formula for these matrix elements exists, and WKB expansions involve unsatisfactory (for our purpose) remainder terms. Nevertheless, there exist explicit formulas for $H_{j}$ expressed in terms of coherent states, which, together with a formulation of Weyl calculus involving Wigner operators (see below), will allow us to conclude. The proof is a bit involved, and we split it in several steps.

\subsection{Hermite as exact Gaussian quasimodes. We first take $d=1$.}

From the formula for the generating function of the Hermite polynomials $\left\{h_{n}^{p}\right\}_{n=0, \ldots}$, namely

$$
\sum_{n=0}^{\infty} h_{n}^{p}(x) \frac{z^{n}}{n !}=e^{2 x z-z^{2}},\left\|h_{n}^{p}\right\|_{L^{2}\left(\mathbf{R}, e^{-x^{2}} d x\right)}=\pi^{\frac{1}{4}} \sqrt{2^{n} n !}
$$

valid for all $z \in \mathbf{C}$, we get easily that the normalized eigenfunctions of the harmonic oscillator $h_{j}, j=0, \ldots$ satisfy

$$
\sum_{j=0}^{\infty} h_{j}(x) \frac{\left(\frac{z}{\sqrt{\hbar}}\right)^{j} e^{-\frac{|z|^{2}}{2 \hbar}}}{\sqrt{j !}}=(\pi \hbar)^{-\frac{1}{4}} e^{-\frac{z^{2}-2 \sqrt{2} z x+x^{2}}{2 \hbar}} e^{-\frac{|z|^{2}}{2 \hbar}}:=g^{z}(x) .
$$

Note that $\left\|g^{z}\right\|_{L^{2}(\mathbf{R}, d x)}=1$. 
We get that, for any choice of $z \in \mathbf{C}, z \neq 0$,

$$
(2 \pi)^{-\frac{3}{4}} \sqrt{\frac{|z|}{\sqrt{\hbar}}} \int_{0}^{2 \pi} g^{e^{i t} z} e^{-i j t} d t=\sqrt{\frac{\left(\frac{|z|^{2}}{\hbar}\right)^{j} e^{-\frac{|z|^{2}}{\hbar}} \sqrt{2 \pi \frac{|z|^{2}}{\hbar}}}{j !}} h_{j}
$$

so that

$$
\begin{aligned}
h_{j} & =\sqrt{\frac{j !}{\left(\frac{|z|^{2}}{\hbar}\right)^{j} e^{-\frac{|z|^{2}}{\hbar}} \sqrt{2 \pi \frac{|z|^{2}}{\hbar}}}}(2 \pi)^{-\frac{3}{4}} \sqrt{\frac{|z|}{\sqrt{\hbar}}} \int_{0}^{2 \pi} g^{e^{i t} z} e^{-i j t} d t \\
& :=C_{j}(z)(2 \pi)^{-\frac{3}{4}} \sqrt{\frac{|z|}{\sqrt{\hbar}}} \int_{0}^{2 \pi} g^{e^{i t} z} e^{-i j t} d t
\end{aligned}
$$

for all $z \neq 0 \in \mathbf{C}$. In particular, taking now $|z|^{2}=(j+1 / 2) \hbar$,

$$
\bar{C}_{j}:=C_{j}(\sqrt{(j+1 / 2) \hbar})=\sqrt{\frac{j !}{\sqrt{2 \pi j} j^{j} e^{-j}}}<1 \text { and } \sim 1 \text { as } j \rightarrow \infty,
$$

since $\sqrt{2 \pi(j+1 / 2)}(j+1 / 2)^{j} e^{-(j+1 / 2)}>\sqrt{2 \pi j} j^{j} e^{-j}$ and $\sqrt{2 \pi(j+1 / 2)}(j+1 / 2)^{j} e^{-(j+1 / 2)} \sim$ $\sqrt{2 \pi j} j^{j} e^{-j}$ as $j \rightarrow \infty$.

The same way,

$$
h_{j^{\prime}}=C_{j^{\prime}}\left(\sqrt{\left(j^{\prime}+1 / 2\right)}\right)(2 \pi)^{-3 / 4}\left(j^{\prime}+1 / 2\right)^{1 / 4} \int_{0}^{2 \pi} g^{e^{i \theta} \sqrt{\left(j^{\prime}+1 / 2\right) \hbar}} e^{-i j^{\prime} \theta} d \theta,
$$

but also $\quad h_{j^{\prime}}=C_{j^{\prime}}(\sqrt{(j+1 / 2)})(2 \pi)^{-3 / 4}(j+1 / 2)^{1 / 4} \int_{0}^{2 \pi} g^{e^{i \theta} \sqrt{(j+1 / 2) \hbar}} e^{-i j^{\prime} \theta} d \theta$.

Therefore $\quad h_{j^{\prime}} \sim \sqrt{\frac{j^{\prime} !}{j !}}(2 \pi)^{-3 / 4}(j+1 / 2)^{j-j^{\prime}+1 / 4} \int_{0}^{2 \pi} g^{e^{i \theta} \sqrt{(j+1 / 2) \hbar}} e^{-i j^{\prime} \theta} d \theta$,

since $\sqrt{2 \pi j} j^{j} e^{-j} \sim \sqrt{2 \pi(j+1 / 2)} j^{j} e^{-(j+1 / 2)}$ as $j \rightarrow \infty$.

Therefore, for any bounded operator $A$,

$$
\left\langle j^{\prime}|A| j\right\rangle=\frac{\bar{C}_{j} \bar{C}_{j^{\prime}}}{(2 \pi)^{\frac{3}{e}}}(j+1 / 2)^{1 / 4}\left(j^{\prime}+1 / 2\right)^{1 / 4} \int_{0}^{2 \pi} d s^{\prime} \int_{0}^{2 \pi} d s e^{i\left(j^{\prime} s^{\prime}-j s\right)}\left(g^{e^{i s^{\prime}} \sqrt{j^{\prime} \hbar}}, A g^{i s} \sqrt{j \hbar}\right) .
$$

but also

$$
\left\langle j^{\prime}|A| j\right\rangle=\frac{\bar{C}_{j} C_{j^{\prime}}(\sqrt{(j+1 / 2) \hbar})}{(2 \pi)^{\frac{3}{e}}}(j+1 / 2)^{1 / 2} \int_{0}^{2 \pi} d s^{\prime} \int_{0}^{2 \pi} d s e^{i\left(j^{\prime} s^{\prime}-j s\right)}\left(g^{e^{i s^{\prime}} \sqrt{j \hbar}}, A g^{e^{i s} \sqrt{j \hbar}}\right) .
$$

Here we have used the Dirac notation, and $\left\langle j^{\prime}|A| j\right\rangle$ is meant for $\left(h_{j^{\prime}}, A h_{j}\right)_{L^{2}(\mathbf{R}, d x)}$. 
In particular,

$$
\left|\left\langle j^{\prime}|A| j\right\rangle\right| \leq \frac{(j+1 / 2)^{1 / 4}\left(j^{\prime}+1 / 2\right)^{1 / 4}}{(2 \pi)^{\frac{3}{2}}}\left|\int_{\mathbf{T}^{2}} d s^{\prime} d s e^{i\left(j^{\prime} s^{\prime}-j s\right)}\left(g^{e^{i s^{\prime}} \sqrt{\left(j^{\prime}+\frac{1}{2}\right) \hbar}}, A g^{e^{i s} \sqrt{\left(j+\frac{1}{2}\right) \hbar}}\right)\right| .
$$

but also

$$
\begin{aligned}
\left|\left\langle j^{\prime}|A| j\right\rangle\right| & \leq(2 \pi)^{-\frac{3}{2}} \sqrt{\frac{j^{\prime} !}{j !}(j+1 / 2)^{j-j^{\prime}+1}}\left|\int_{\mathbf{T}^{2}} d s^{\prime} d s e^{i\left(j^{\prime} s^{\prime}-j s\right)}\left(g^{e^{i s^{\prime}} \sqrt{\left(j^{\prime}+\frac{1}{2}\right) \hbar}}, A g^{i s} \sqrt{\left(j+\frac{1}{2}\right) \hbar}\right)\right| \\
(54) & \leq \frac{e^{\frac{\left(j-j^{\prime}\right)^{2}}{j+1 / 2}}(j+1 / 2)^{1 / 2}}{(2 \pi)^{\frac{3}{2}}} \mid \int_{\mathbf{T}^{2}} d s^{\prime} d s e^{i\left(j^{\prime} s^{\prime}-j s\right)}\left(g^{e^{i s^{\prime}} \sqrt{\left(j^{\prime}+\frac{1}{2}\right) \hbar}}, A g^{\left.e^{i s} \sqrt{\left(j+\frac{1}{2}\right) \hbar}\right)} \mid\right.
\end{aligned}
$$

Indeed, if $j^{\prime}=j+k, k \geq 0$ (the case $k<0$ is done the same way),

$$
\frac{j^{\prime} !}{j !}(j+1 / 2)^{j-j^{\prime}} \leq \frac{(j+1 / 2+k)^{k}}{(j+1 / 2)^{k}} \leq\left(1+\frac{k}{j+1 / 2}\right)^{k} \leq e^{\frac{k^{2}}{j+1 / 2}}
$$

We'll estimate later $\left|\left\langle j^{\prime}|A| j\right\rangle\right|$ by (54) for $e^{\frac{\left(j-j^{\prime}\right)^{2}}{j+1 / 2}} \leq 1$ and by (53) for $e^{\frac{\left(j-j^{\prime}\right)^{2}}{2}}>1$.

The extension to the $d$-dimensional case is straightforward, by tensorial factorization.

7.2. Weyl calculus through Wigner operators. Let us suppose now that $A$ is a Hilbert-Schmidt operator of Wigner function $a \in L^{2}\left(\mathbf{R}^{d}\right)$. Note that this is to say that, in a weak sense,

$$
A=\int_{\mathbf{C}^{d}} d^{2} z a(z) W(-z) I W(z):=\operatorname{Wig}(a)
$$

where the Wigner operators $W(z)$ are the unitary operators on $L^{2}\left(\mathbf{R}^{d}\right)$ defined through (denoting $z=(q, p)$ )

$$
W(q, p) f(x)=f(x-q) e^{-i \frac{p x}{\hbar}} e^{-i \frac{p q}{4}} \text { and } \operatorname{If}(x):=f(-x) .
$$

Indeed the integral kernel of $W(-z) I W(z)$ is $\delta(-x-2 q-y) e^{i \frac{p\left(2 x-\frac{q}{2}\right)}{\hbar}}$ so that the integral kernel of $A=\int_{\mathbf{C}^{d}} d^{2} z a(z) W(-z) I W(z)$ is

$$
\rho_{A}(x, y)=\int_{\mathbf{R}^{d}} a\left(\frac{x+y}{2}, \xi\right) e^{i \frac{\xi(x-y)}{\hbar}} d \xi
$$

which is the well-known Weyl quantization formula expressed on the Wigner function (let us recall that the latter is nothing but the Weyl symbol divided by $(2 \pi \hbar)^{d}$ ).

We get easily that

$$
\begin{aligned}
\left(g^{z^{\prime}}, W\left(-z_{0}\right) I W\left(z_{0}\right) g^{z}\right) & =e^{\frac{\bar{z} z_{0}-\bar{z}_{0} z}{\sqrt{2} \hbar}} e^{-\frac{\bar{z}^{\prime}\left(z+\sqrt{2} z_{0}\right)}{\hbar}} e^{-\frac{\left|z^{\prime}\right|}{2 \hbar}} e^{-\frac{\left|z+\sqrt{2} z_{0}\right|}{2 \hbar}} \\
& =e^{-\frac{\left|z^{\prime}\right|^{2}}{2 \hbar}-\frac{|z|^{2}}{2 \hbar}-\frac{\left|z_{0}\right|^{2}}{\hbar}-\frac{\bar{z}^{\prime} z}{\hbar}-\sqrt{2} \frac{\overline{z^{\prime} z_{0}+z z_{0}}}{\hbar}} .
\end{aligned}
$$


so that

$$
\left(g^{z^{\prime}}, W\left(-\frac{z_{0}}{\sqrt{2}}\right) I W\left(\frac{z_{0}}{\sqrt{2}}\right) g^{z}\right)=e^{-\frac{\left|z^{\prime}\right|^{2}}{2 \hbar}-\frac{|z|^{2}}{2 \hbar}-\frac{\left|z_{0}\right|^{2}}{2 \hbar}-\frac{\overline{z^{\prime}} z}{\hbar}-\frac{\overline{z^{\prime}} z_{0}+z \overline{z_{0}}}{\hbar}}
$$

Note that

$$
\Re\left(\frac{\left|z^{\prime}\right|^{2}}{2}+\frac{|z|^{2}}{2}+\frac{\left|z_{0}\right|^{2}}{2}+\overline{z^{\prime}} z+\overline{z^{\prime}} z_{0}+z \overline{z_{0}}\right)=\left|z^{\prime}+z+z_{0}\right|^{2} \geq 0 .
$$

For $d=1$ we get that (53) becomes

$$
\begin{aligned}
\left|\left\langle j^{\prime}|A| j\right\rangle\right| \leq & \frac{(j+1 / 2)^{1 / 4}\left(j^{\prime}+1 / 2\right)^{1 / 4}}{(2 \pi)^{\frac{3}{2}}} B_{j, j^{\prime}} \\
B_{j, j^{\prime}}:= & \left|\int_{S^{1}} d \theta^{\prime}\left(\int_{\mathbf{R}^{2}} d^{2} z \int_{S^{1}} d \theta a\left(\frac{z}{\sqrt{2}}\right) e^{-\frac{\phi_{j, j^{\prime}}\left(z, \bar{z}, \theta, \theta^{\prime}\right)}{\hbar}}\right) e^{i\left(j^{\prime}-j\right) \theta^{\prime}}\right| \\
\phi_{j, j^{\prime}}\left(z, \bar{z}, \theta, \theta^{\prime}\right):= & \frac{\left(j+\frac{1}{2}\right) \hbar}{2}+\frac{\left(j^{\prime}+\frac{1}{2}\right) \hbar}{2}+\frac{z \bar{z}}{2}+\sqrt{\left(j+\frac{1}{2}\right)\left(j^{\prime}+\frac{1}{2}\right)} \hbar e^{i\left(\theta-\theta^{\prime}\right)} \\
& +\bar{z} \sqrt{\left(j+\frac{1}{2}\right) \hbar} e^{i \theta}+z \sqrt{\left(j^{\prime}+\frac{1}{2}\right) \hbar} e^{-i \theta^{\prime}}+i j \hbar\left(\theta-\theta^{\prime}\right) .
\end{aligned}
$$

and (54) becomes

$$
\begin{aligned}
\left|\left\langle j^{\prime}|A| j\right\rangle\right| \leq & \frac{e^{\frac{\left(j-j^{\prime}\right)^{2}}{j+1 / 2}}(j+1 / 2)^{1 / 2}}{(2 \pi)^{\frac{3}{2}}} A_{j, j^{\prime}} \\
A_{j, j^{\prime}}:= & \left|\int_{S^{1}} d \theta^{\prime}\left(\int_{\mathbf{R}^{2}} d^{2} z \int_{S^{1}} d \theta a\left(\frac{z}{\sqrt{2}}\right) e^{-\frac{\phi_{j}\left(z, \bar{z}, \theta, \theta^{\prime}\right)}{\hbar}}\right) e^{i\left(j^{\prime}-j\right) \theta^{\prime}}\right| \\
\phi_{j}\left(z, \bar{z}, \theta, \theta^{\prime}\right):= & \left(j+\frac{1}{2}\right) \hbar+\frac{z \bar{z}}{2}+\left(j+\frac{1}{2}\right) \hbar e^{i\left(\theta-\theta^{\prime}\right)} \\
& +\bar{z} \sqrt{\left(j+\frac{1}{2}\right) \hbar} e^{i \theta}+z \sqrt{\left(j+\frac{1}{2}\right) \hbar} e^{-i \theta^{\prime}}+i j \hbar\left(\theta-\theta^{\prime}\right) .
\end{aligned}
$$

Performing in (57) and (60) first the change of variable $z \rightarrow z e^{i \theta^{\prime}}$ and then the one $\theta \rightarrow \theta+\theta^{\prime}$ we get easily that, after definition (14)

$$
\begin{aligned}
& B_{j, j^{\prime}}=\left|\int_{\mathbf{R}^{2}} d^{2} z \int_{S^{1}} d \theta a_{j-j^{\prime}}\left(\frac{z}{\sqrt{2}}\right) e^{-\frac{\Phi_{j, j^{\prime}}(z, \bar{z}, \theta)}{\hbar}}\right| \\
& A_{j \cdot j^{\prime}}=\left|\int_{\mathbf{R}^{2}} d^{2} z \int_{S^{1}} d \theta a_{j-j^{\prime}}\left(\frac{z}{\sqrt{2}}\right) e^{-\frac{\Phi_{j}(z, \bar{z}, \theta)}{\hbar}}\right|
\end{aligned}
$$

(64) with $\Phi_{j, j^{\prime}}(z, \bar{z}, \theta):=\frac{\left(j+\frac{1}{2}\right) \hbar}{2}+\frac{\left(j^{\prime}+\frac{1}{2}\right) \hbar}{2}+\frac{z \bar{z}}{2}+\sqrt{\left(j+\frac{1}{2}\right)\left(j^{\prime}+\frac{1}{2}\right)} \hbar e^{i \theta}$

$$
+\bar{z} \sqrt{\left(j+\frac{1}{2}\right) \hbar} e^{i \theta}+z \sqrt{\left(j^{\prime}+\frac{1}{2}\right) \hbar}+i j \hbar \theta
$$

(65) and

$$
\begin{aligned}
\Phi_{j}(z, \bar{z}, \theta):= & \Phi_{j, j}(z, \bar{z}, \theta)=\left(j+\frac{1}{2}\right) \hbar+\frac{z \bar{z}}{2}+\left(j+\frac{1}{2}\right) \hbar e^{i \theta} \\
& +\bar{z} \sqrt{\left(j+\frac{1}{2}\right) \hbar} e^{i \theta}+z \sqrt{\left(j+\frac{1}{2}\right) \hbar}+i j \hbar \theta .
\end{aligned}
$$


Let us recall that

$$
\Re \Phi_{j, j^{\prime}}(z, \bar{z}, \theta)=\left|\sqrt{\left(j+\frac{1}{2}\right) \hbar} e^{i \theta_{l}}+\sqrt{\left(j^{\prime}+\frac{1}{2}\right) \hbar}+z\right|^{2} \geq 0
$$

so that, since $\Phi_{j}=\Phi_{j, j}$

$$
A_{j, j^{\prime}}, B_{j, j^{\prime}} \leq \frac{(2 \pi)^{3} \hbar}{2}\left\|a_{j-j^{\prime}}\right\|_{L^{\infty}\left(\mathbf{R}^{2 d}\right)}
$$

Since $\Phi_{j, j^{\prime}}^{d}$ is a sum of one dimensional contributions, we will treat the one dimensional case. The (straightforward) extension to $d$ dimensions will be done in the next section.

\subsection{The case $d=1$.}

Note that for $d=1,(15)$ reads, for any $K, M \in \mathbf{R}^{+}, N \in \mathbf{N}$

$$
\sup _{0 \leq|\alpha| \leq N}\left|\hbar^{\frac{|\alpha|}{2}} D_{z}^{\alpha} a_{k}(z)\right|, \leq\|a\|_{M, K, N}\left(|z|^{2}+1\right)^{-M}(|k|+1)^{-K}
$$

7.3.1. Matrix elements estimates. In this section we prove the following crucial result.

Proposition 7.1. Let $A$ by a Hilbert-Schmidt operator (say).

Then for all $M, \alpha, \tau, \mu, n \geq 0$,

$$
\left|\left\langle j^{\prime}|A| j\right\rangle\right| \leq D_{\mu, n} \frac{2 \pi \hbar\left\|W_{\hbar}[A]\right\|_{M, 1+\epsilon+\tau, n}}{|| j^{\prime}-j\left|+\frac{1}{2}\right| \min (1+\alpha+\epsilon, 1+\epsilon+\tau)\left(\left(j+\frac{1}{2}\right) \hbar+\frac{1}{2}\right)^{\min \left(\frac{\tau-\alpha-1}{2}, \mu-\frac{3}{2}, M, \frac{n-1}{2}\right)}} .
$$

where $D_{\mu, n}$ is given below by $(84)$.

Proof. The desired estimates of $\left|\left\langle j^{\prime}|A| j\right\rangle\right|$, expressed as in (53), that is (56) or (59), will be obtained by different methods, depending on the size of $\left|j^{\prime}-j\right|$.

(1) for $\left|j^{\prime}-j\right|^{2}>j+\frac{1}{2}$, we will use (57) and (62), together with (67), to estimate $\left|\left\langle j^{\prime}|A| j\right\rangle\right|$.

(2) for $\left|j^{\prime}-j\right|^{2} \leq j+\frac{1}{2}$, we will use (60) and (63): we will perform a smooth partition of the domain of integration in $|z|$ in the integral present in the expression $A_{j^{\prime}, j}$ in $(63)$.

(a) for $|z| \geq \frac{5}{2} \sqrt{\left(j+\frac{1}{2}\right) \hbar}$, the decay given by the negative imaginary part of the phase will be enough.

(b) for $\sqrt{\left(j+\frac{1}{2}\right) \hbar} \leq|z| \leq 3 \sqrt{\left(j+\frac{1}{2}\right) \hbar}$, we will have two cases:

(i) $\left|j^{\prime}-j\right|>0$, the desired estimates will be obtained by a brutal estimate of the integral of $\left|a_{j^{\prime}-j}\right|$ on the domain.

(ii) $j+j^{\prime}$, the stationary phase lemma applied to the integral on the domain will give the needed estimate.

(c) for $|z| \leq \frac{3}{2} \sqrt{\left(j+\frac{1}{2}\right) \hbar}$, the non stationary phase Lemma will apply and give the result. 
Note that since $A$ is self-adjoint, one can restrict to the case $j^{\prime} \leq j$ without loss of generality.

(1) Suppose $\left|j^{\prime}-j\right|^{2}>j$. By (57), (62) and (67) we get for any $\alpha$

$$
\begin{aligned}
\left|\left\langle j^{\prime}|A| j\right\rangle\right| & \leq \frac{(2 \pi)^{\frac{3}{2} \hbar}}{2}\left(j+\frac{1}{2}\right)^{1 / 4}\left(j^{\prime}+\frac{1}{2}\right)^{1 / 4}\left\|a_{j-j^{\prime}}\right\|_{L^{\infty}\left(\mathbf{R}^{2 d}\right)} \\
& \leq \frac{(2 \pi)^{\frac{3}{2} \hbar}}{2}\|a\|_{M, 1+\tau+\epsilon, N}\left(j+\frac{1}{2}\right)^{1 / 4}\left(j^{\prime}+\frac{1}{2}\right)^{1 / 4}\left(\left|j-j^{\prime}\right|+1\right)^{-1-\epsilon-\tau} \\
& \leq \frac{(2 \pi)^{\frac{3}{2} \hbar}}{2}\|a\|_{M, 1+\tau+\epsilon, N}\left(j+\frac{1}{2}\right)^{1 / 2}\left(\left(\left|j-j^{\prime}\right|+1\right)^{-1-\alpha-\epsilon}(\sqrt{j}+1)^{-\tau+\alpha}\right. \\
& \leq \frac{(2 \pi)^{\frac{3}{2} \hbar}}{2}\|a\|_{M, 1+\tau+\epsilon, N}\left(\left|j-j^{\prime}\right|+1\right)^{-1-\alpha-\epsilon}(j+1)^{-(\tau-\alpha-1) / 2} \\
& \leq \frac{(2 \pi)^{\frac{3}{2}}}{2} \hbar\|a\|_{M, 1+\tau+\epsilon, N}\left(\left|j-j^{\prime}\right|+1\right)^{-1-\alpha-\epsilon}\left(\left(j+\frac{1}{2}\right) \hbar+\frac{1}{2}\right)^{-(\tau-\alpha-1) / 2} .
\end{aligned}
$$

(2) We now consider the case $\left|j^{\prime}-j\right|^{2} \leq j+\frac{1}{2}$. We will use (60) which becomes

$$
\left|\left\langle j^{\prime}|A| j\right\rangle\right| \leq \frac{(j+1 / 2)^{1 / 2}}{(2 \pi)^{\frac{3}{2}}} A_{j, j^{\prime}}
$$

We first perform a change of variable $z \rightarrow\left(\left(j+\frac{1}{2}\right) \hbar\right)^{1 / 2} z$ in the integral inside the definition of $A_{j, j^{\prime}}$ in (60). We get

$$
\begin{aligned}
A_{j, j^{\prime}} & =\left|\int_{\mathbf{R}^{2}} d^{2} z \int_{S^{1}} d \theta a_{j-j^{\prime}}\left(\frac{z}{\sqrt{2}}\right) e^{-\frac{\Phi_{j}\left(z, \bar{z}, \theta^{\prime}\right)}{\hbar}}\right| \\
& =\left(j+\frac{1}{2}\right) \hbar\left|\int_{\mathbf{R}^{2}} d^{2} z \int_{S^{1}} d \theta a_{j-j^{\prime}}\left(\frac{\sqrt{\left(j+\frac{1}{2}\right) \hbar}}{\sqrt{2}} z\right) e^{-\left(j+\frac{1}{2}\right) \frac{\Phi(z, \bar{z}, \theta)}{\hbar}}\right| .
\end{aligned}
$$

with

$$
\Phi(z, \bar{z}, \theta)=1+\frac{z \bar{z}}{2}+e^{i \theta}+\bar{z} e^{i \theta}+z+i \theta .
$$

We first perform a smooth decomposition of the identity $1=\chi^{\leq}+\chi^{=}+\chi^{\geq}$, such that,

$$
\left\{\begin{array}{l}
\sup \chi^{\leq}=\left\{z,|z| \leq \frac{3}{2}\right\} \\
\sup \chi^{=}=\{z, 1 \leq|z| \leq 3\} \\
\sup \chi^{\geq}=\left\{z,|z| \geq \frac{5}{2}\right\}
\end{array}\right.
$$

and decompose $a_{j-j^{\prime}}$ in three terms $a_{j-j^{\prime}}^{\bullet}, \bullet \in\{\leq,=, \geq\}$ :

$$
a_{j-j^{\prime}}^{\bullet}\left(\frac{\sqrt{\left.\left(j+\frac{1}{2}\right)\right) \hbar z}}{\sqrt{2}}\right):=\chi^{\bullet}(z) a_{j-j^{\prime}}\left(\frac{\sqrt{\left.\left(j+\frac{1}{2}\right)\right) \hbar z}}{\sqrt{2}}\right) .
$$


Note that, since the $\chi^{\bullet}$ are smooth and constant for $|z| \geq 3$,

$$
\left|D_{z}^{\alpha} a_{j-j^{\prime}}^{\bullet}\left(\frac{\sqrt{\left.\left(j+\frac{1}{2}\right)\right) \hbar} z}{\sqrt{2}}\right)\right| \leq C_{\chi,|\alpha|} \sum_{|\beta| \leq|\alpha|}\left|D_{z}^{\beta} a_{j-j^{\prime}}\left(\frac{\sqrt{\left.\left(j+\frac{1}{2}\right)\right) \hbar} z}{\sqrt{2}}\right)\right|
$$

with

$$
C_{\chi,|\alpha|}=2^{|\alpha|} \sum_{\substack{\bullet \in\{\leq,=, \geq\} \\|\beta| \leq|\alpha|}}\left\|D_{z}^{\beta} \chi^{\bullet}\right\|_{L^{\infty}\left(\mathbf{R}^{2}\right)} .
$$

Of course, $C_{\chi, 0}=1$.

(a) $\bullet=\geq$.

By (66), $\Re \Phi\left(z, \bar{z} \theta, \theta^{\prime}\right) \geq \frac{1}{4}$ for $z$ in the support of $a_{j-j^{\prime}}^{\geq}$, the latter gives a contribution to $A_{j, j^{\prime}}$ (which is linear in $a$ ) in (63) equal to

$$
\begin{aligned}
& A_{j, j^{\prime}}^{\geq} \leq 2(2 \pi)^{2}\left(j+\frac{1}{2}\right) \hbar\left\|a_{j-j^{\prime}}^{\geq}\right\|_{L^{\infty}\left(\mathbf{R}^{2 d}\right)} \int_{|z| \geq \frac{5}{2}} e^{-\left(j+\frac{1}{2}\right)\left|z+e^{i \theta}+e^{-i \theta^{\prime}}\right|^{2}} d z d \bar{z} d \theta d \theta^{\prime} . \\
& \leq 2(2 \pi)^{2}\left(j+\frac{1}{2}\right) \hbar\left\|a_{j-j^{\prime}}^{\geq}\right\|_{L^{\infty}\left(\mathbf{R}^{2 d}\right)} e^{-\left(j+\frac{1}{2}\right) / 4} \int_{|z| \geq \frac{5}{2}} e^{-\left(j+\frac{1}{2}\right)\left(\left|z+e^{i \theta}+e^{-i \theta^{\prime}}\right|^{2}-\frac{1}{4}\right)} d z d \bar{z} d \theta d \theta^{\prime} . \\
& \leq 2(2 \pi)^{2}\left(j+\frac{1}{2}\right) \hbar\left\|a_{j-j^{\prime}}^{\geq}\right\|_{L^{\infty}\left(\mathbf{R}^{2 d}\right)} e^{-\left(j+\frac{1}{2}\right) / 4} \int_{|z| \geq \frac{5}{2}} e^{-\frac{1}{2}\left(\left|z+e^{i \theta}+e^{-i \theta^{\prime}}\right|^{2}-\frac{1}{4}\right)} d z d \bar{z} d \theta d \theta^{\prime} \\
& \leq 2(2 \pi)^{2}\left(j+\frac{1}{2}\right) \hbar\left\|a_{j-j^{\prime}}\right\|_{L^{\infty}\left(\mathbf{R}^{2 d}\right)} e^{-\left(j+\frac{1}{2}\right) / 4}(2 \pi)^{2} \int_{\mathbf{C}} e^{\frac{1}{8}} e^{-\frac{1}{2}\left(|z|^{2}\right.} d z d \bar{z} \\
& \leq 2(2 \pi)^{5} e^{\frac{1}{8}}\left(j+\frac{1}{2}\right) \hbar\|a\|_{M, 1+\epsilon+\tau, N}\left(\left|j-j^{\prime}\right|+1\right)^{-1-\epsilon-\tau} e^{-\left(j+\frac{1}{2}\right) / 4} \text {. }
\end{aligned}
$$

so that, by (68) again,

$\left|\left\langle j^{\prime}\left|\operatorname{Wig}\left(a^{\geq}\right)\right| j\right\rangle\right| \leq 2(2 \pi)^{\frac{7}{2}} e^{\frac{1}{8}} \hbar\|a\|_{M, 1+\epsilon+\tau, N}\left(\left|j-j^{\prime}\right|+1\right)^{-1-\epsilon-\tau}\left(j+\frac{1}{2}\right)^{\frac{3}{2}} e^{-\frac{j+\frac{1}{2}}{4}}$

$$
\leq 2(2 \pi)^{\frac{7}{2}} e^{\frac{1}{8}}\left(\frac{4 \mu}{e}\right)^{\mu} \hbar\|a\|_{M, 1+\epsilon+\tau, N}\left(\left|j^{\prime}-j\right|+1\right)^{-1-\epsilon-\tau}\left(\left(j+\frac{1}{2}\right) \hbar+\frac{1}{2}\right)^{\frac{3}{2}-\mu}
$$

for all $\mu>\frac{1}{2}$.

(b) $\bullet==$

We will use the stationary phase Lemma for estimating $A_{j, j}^{\bar{j}}$ given by (63). We get

$$
\begin{aligned}
\partial_{z} \Phi(z, \bar{z}, \theta) & =\frac{\bar{z}}{2}+1 \\
\partial_{\bar{z}} \Phi(z, \bar{z}, \theta) & =\frac{z}{2}+e^{i \theta^{\prime \prime}} \\
\partial_{\theta} \Phi(z, \bar{z}, \theta) & =i\left(e^{i(\theta)}+\bar{z} e^{i \theta}+1\right),
\end{aligned}
$$

so that the critical point of $\Phi$ is at $\theta=0, z=-2$ and, at the critical point, 


$$
\Phi=0, d^{2} \Phi=\left(\begin{array}{ccc}
0 & 1 / 2 & 0 \\
1 / 2 & 0 & 1 \\
0 & 1 & 1
\end{array}\right) \text { and } \operatorname{det}\left(d^{2} \Phi\right)=-1 / 4
$$

The stationary phase Lemma (with quadratic phase) gives immediately that, as $j \rightarrow \infty$ (independently of $\hbar$ ),

$$
\begin{aligned}
A_{j, j}^{=} \leq & \frac{2}{\pi^{\frac{3}{2}}} \frac{\left(j+\frac{1}{2}\right) \hbar}{\left(j+\frac{1}{2}\right)^{3 / 2}}\left(\left|a_{j^{\prime}-j}\left(\sqrt{2\left(j+\frac{1}{2}\right) \hbar}\right)\right|\right. \\
& \left.+\frac{1}{16} \frac{1}{j+\frac{1}{2}} \sup _{|\alpha|=2}\left|D_{z}^{\alpha} a_{j^{\prime}-j}^{\overline{1}-|z| \leq 3}\left(\sqrt{\frac{\left(j+\frac{1}{2}\right) \hbar}{2}} z\right)\right|\right), \\
\leq & \frac{2}{\pi^{\frac{3}{2}}}\left(1+\frac{C_{\chi, 2}}{4}\right)\|a\|_{M, 1+\epsilon+\tau, N}\left(\left|j-j^{\prime}\right|+1\right)^{-1-\epsilon-\tau}\left(\left(j+\frac{1}{2}\right) \hbar+1\right)^{-M}
\end{aligned}
$$

since, by (75),

$$
\begin{aligned}
& \left|D_{z}^{\alpha} a_{j^{\prime}-j}^{=}\left(\sqrt{\frac{\left(j+\frac{1}{2}\right) \hbar}{2}} z\right)\right| \\
\leq & C_{\chi,|\alpha|} \sum_{|\beta| \leq|\alpha|}\left|D_{z}^{\beta} a_{j-j^{\prime}}\left(\frac{\sqrt{\left.\left(j+\frac{1}{2}\right)\right) \hbar} z}{\sqrt{2}}\right)\right| \\
\leq & C_{\chi,|\alpha|} \sum_{|\beta| \leq|\alpha|}\left(\frac{\sqrt{\left.\left(j+\frac{1}{2}\right)\right)}}{\sqrt{2}}\right)^{|\beta|}\left|\left(\left(\sqrt{\hbar} D_{z}\right)^{\beta} a_{j-j^{\prime}}\right)\left(\frac{\sqrt{\left.\left(j+\frac{1}{2}\right)\right) \hbar z}}{\sqrt{2}}\right)\right| \\
\leq & C_{\chi,|\alpha|} 2^{\frac{|\alpha|}{2}}\left(j+\frac{1}{2}\right)^{\frac{|\alpha|}{2}} \sum_{|\beta| \leq|\alpha|}\left(\frac{\sqrt{\left.\left(j+\frac{1}{2}\right)\right)}}{\sqrt{2}}\right)^{|\beta|}\left|\left(\left(\sqrt{\hbar} D_{z}\right)^{\beta} a_{j-j^{\prime}}\right)\left(\frac{\sqrt{\left.\left(j+\frac{1}{2}\right)\right) \hbar z}}{\sqrt{2}}\right)\right|
\end{aligned}
$$

$(78) \leq\left. C_{\chi,|\alpha|}\right|^{\frac{|\alpha|}{2}}\left(j+\frac{1}{2}\right)^{\frac{|\alpha|}{2}}\|a\|_{M, 1+\epsilon+\tau, N}\left(\left|j-j^{\prime}\right|+1\right)^{-1-\epsilon-\tau}\left(\left(j+\frac{1}{2}\right) \hbar|z|^{2}+1\right)^{-M}$

$$
\leq C_{\chi,|\alpha|^{2}} 2^{\frac{|\alpha|}{2}}\left(j+\frac{1}{2}\right)^{\frac{|\alpha|}{2}}\|a\|_{M, 1+\epsilon+\tau, N}\left(\left|j-j^{\prime}\right|+1\right)^{-1-\epsilon-\tau}\left(\left(j+\frac{1}{2}\right) \hbar+1\right)^{-M},|z| \geq 1 .
$$

Here we have use the fact that $\sqrt{j+\frac{1}{2}} \leq 2\left(j+\frac{1}{2}\right), j=0.1, \ldots$

Therefore, by (68),

$$
\begin{aligned}
\left|\left\langle j^{\prime}\left|\operatorname{Wig}\left(a^{=}\right)\right| j\right\rangle\right| & \leq \frac{1}{(2 \pi)^{\frac{3}{2}}} \frac{2}{\pi^{\frac{3}{2}}}\left(1+\frac{C_{\chi, 2}}{4}\right) \hbar\|a\|_{M, 1+\epsilon+\tau, N}\left(\left|j-j^{\prime}\right|+1\right)^{-1-\epsilon-\tau}\left(\left(j+\frac{1}{2}\right) \hbar+1\right)^{-M} \\
(79) & \leq \frac{1+\frac{C_{\chi, 2}}{4}}{\sqrt{2} \pi^{3}} \hbar\|a\|_{M, 1+\epsilon+\tau, N}\left(\left|j-j^{\prime}\right|+1\right)^{-1-\epsilon-\tau}\left(\left(j+\frac{1}{2}\right) \hbar+1\right)^{-M}
\end{aligned}
$$

$(\mathrm{c}) \bullet=\leq$ 
Finally, we will estimate the contribution given by $a_{j-j^{\prime}}^{\leq}$by first estimating the integral $\int d^{2} z a_{j-j^{\prime}}^{\leq}\left(\frac{\sqrt{\left(j+\frac{1}{2}\right) \hbar} z}{\sqrt{2}}\right) e^{-\left(j+\frac{1}{2}\right) \Phi(z, \bar{z}, \theta)}$ pointwise in $\theta$ by the non-stationary phase method. Indeed,

Therefore, for $z \in \sup a_{j-j^{\prime}}^{\leq}$

$$
\begin{aligned}
& \partial_{z} \Phi=\frac{\bar{z}}{2}+e^{-i \theta^{\prime}} \\
& \partial_{\bar{z}} \Phi=\frac{z}{2}+e^{i \theta}
\end{aligned}
$$

Using now that

$$
\frac{1}{2} \leq\left|D_{z} \Phi\right| \leq \frac{7}{2}
$$

$$
e^{-\left(j+\frac{1}{2}\right) \Phi(z, \bar{z}, \theta)}=\frac{1}{j+\frac{1}{2}} \frac{1}{\left|D_{z} \Phi\right|^{2}} D_{z} \Phi \cdot D_{z} e^{-\left(j+\frac{1}{2}\right) \Phi(z, \bar{z}, \theta)}
$$

Let

$$
D_{\Phi}:=D_{z} \cdot\left(D_{z} \Phi\right)\left|D_{z} \Phi\right|^{-2}=\left(\left|D_{z} \Phi\right|^{-2} D_{z} \Phi \cdot D_{z}\right)^{*} .
$$

We get by integration by part that, for any $n \in \mathbf{N}$,

$$
\begin{aligned}
A_{j, j^{\prime}} & \leq\left(j+\frac{1}{2}\right) \hbar\left(j+\frac{1}{2}\right)^{-n}\left|\int D_{\Phi}^{n}\left(a_{j-j^{\prime}}^{\leq}\left(\frac{\sqrt{\left(j+\frac{1}{2}\right) \hbar z}}{\sqrt{2}}\right)\right) e^{-\left(j+\frac{1}{2}\right) \Phi(z, \bar{z}, \theta)} d^{2} z d \theta\right| \\
& \leq\left(j+\frac{1}{2}\right) \hbar \frac{2 \pi}{\left(j+\frac{1}{2}\right)^{n}} \sup _{\substack{|z| \leq \frac{3}{2} \\
\left(\theta, \theta^{\prime}\right) \in \mathbf{T}^{2}}}\left|D_{\Phi}^{n}\left(a_{j-j^{\prime}}^{\leq}\left(\frac{\sqrt{\left(j+\frac{1}{2}\right) \hbar z}}{\sqrt{2}}\right)\right)\right| \int e^{-\left(j+\frac{1}{2}\right) \Re \Phi\left(z, \bar{z}, \theta, \theta^{\prime}\right)} d^{2} z .
\end{aligned}
$$

One has

$$
\begin{aligned}
& \sup _{\substack{|z| \leq \frac{3}{2} \\
\left(\theta, \theta^{\prime}\right) \in \mathbf{T}^{2}}}\left|D_{\Phi}^{n}\left(a_{j-j^{\prime}}^{\leq}\left(\frac{\sqrt{\left(j+\frac{1}{2}\right) \hbar z}}{\sqrt{2}}\right)\right)\right| \\
\leq & 2^{n} \sup _{\substack{|z| \leq \frac{3}{2} \\
\left(\theta, \theta^{\prime}\right) \in \mathbf{T}^{2}}} \sup _{|\beta|+k \leq n}\left|D^{\beta}\left(\frac{\Phi(z)}{\left|D_{z} \Phi(z)\right|^{2}}\right)^{k}\right| \sum_{|\alpha| \leq n}\left|D_{z}^{\alpha}\left(a_{j-j^{\prime}}^{\leq}\left(\frac{\sqrt{\left(j+\frac{1}{2}\right) \hbar z}}{\sqrt{2}}\right)\right)\right| \\
\leq & 2^{n} C_{\Phi}\left|\sum_{|\alpha| \leq n}\right| D_{z}^{\alpha}\left(a_{j-j^{\prime}}^{\leq}\left(\frac{\sqrt{\left(j+\frac{1}{2}\right) \hbar z}}{\sqrt{2}}\right)\right) \mid \\
\leq & 2^{n} C_{\Phi} C_{\chi, n} 2^{\frac{n}{2}}\left(j+\frac{1}{2}\right)^{\frac{n}{2}}\|a\|_{M, 1+\epsilon+\tau, N}\left(\left|j-j^{\prime}\right|+1\right)^{-1-\epsilon-\tau}
\end{aligned}
$$

by (78).

We have used $\left(j+\frac{1}{2}\right)^{k} \leq 2^{n}\left(j+\frac{1}{2}\right)^{n}, j=0,1, \ldots, 0 \leq k \leq n$ and define

$$
C_{\Phi}=\sup _{\substack{|z| \leq \frac{3}{2} \\\left(\theta, \theta^{\prime}\right) \in \mathbf{T}^{2}}} \sup _{\substack{|\beta|+k \leq n\\}}\left|D^{\beta}\left(\frac{\Phi(z)}{\left|D_{z} \Phi(z)\right|^{2}}\right)^{k}\right| .
$$


Therefore

$$
A_{j, j^{\prime}} \leq 2^{\frac{3}{2} n} C_{\Phi} C_{\chi, n} \frac{2 \pi^{2}}{\left(j+\frac{1}{2}\right)^{\frac{n}{2}}} \hbar\|a\|_{M, 1+\epsilon+\tau, N}\left(\left|j-j^{\prime}\right|+1\right)^{-1-\epsilon-\tau},
$$

so that, for by (59),

$$
\begin{aligned}
& \left|\left\langle j^{\prime}\left|\operatorname{Wig}\left(a_{j^{\prime}-j}^{\leq}\right)\right| j\right\rangle\right| \\
\leq & \frac{2^{\frac{3}{2} n}}{(2 \pi)^{\frac{3}{2}}} C_{\Phi} C_{\chi, n} \frac{2 \pi^{2}}{\left(j+\frac{1}{2}\right)^{\frac{n-1}{2}}} \hbar\|a\|_{M, 1+\epsilon+\tau, N}\left(\left|j-j^{\prime}\right|+1\right)^{-1-\epsilon-\tau} \\
\leq & C_{\Phi} C_{\chi, n} \frac{2^{\frac{3}{2}(n-1)} \pi^{\frac{1}{2}}}{\left(j+\frac{1}{2}\right)^{\frac{n-1}{2}}} \hbar\|a\|_{M, 1+\epsilon+\tau, N}\left(\left|j-j^{\prime}\right|+1\right)^{-1-\epsilon-\tau} \\
\leq & 2^{\frac{n-1}{2}} C_{\Phi} C_{\chi, n} \hbar\|a\|_{M, 1+\epsilon+\tau, N}\left(\left|j-j^{\prime}\right|+1\right)^{-1-\epsilon-\tau}\left(\left(j+\frac{1}{2}\right)+1\right)^{-\frac{n-1}{2}}
\end{aligned}
$$

Adding the estimates given by (70), (77), (79) and (83), we get (69) with

$$
2 \pi D_{\mu, n}=\frac{(2 \pi)^{3 / 2}}{2}+2(2 \pi)^{\frac{7}{2}} e^{\frac{1}{8}}(4 \mu)^{\mu} e^{-\mu}+\frac{1+\frac{C_{\chi, 2}}{4}}{\sqrt{2} \pi^{3}}+2^{\frac{n-1}{2}} C_{\Phi} C_{\chi, n} .
$$

7.3.2. Hypothesis 1 in Theorem 2.1 C Condition 1 in Theorem 2.2.

By Proposition $7.1 \sqrt{R^{i n}}$ satisfies the hypothesis (1) in Theorem 2.2 as soon as

$$
\min (1+\alpha+\epsilon, 1+\epsilon+\tau)=2+\epsilon, \min \left(\frac{\tau-\alpha-1}{2}, \mu-\frac{3}{2}, M, \frac{n-1}{2}\right)=\frac{3}{4}+\epsilon,
$$

that is

and

$$
\alpha=1, \tau=\frac{7}{2}+2 \epsilon, \mu=\frac{9}{4}+\epsilon, M=\frac{3}{4}+\epsilon, n=3
$$

$$
\left\|W_{\hbar}\left[\sqrt{R^{i n}}\right]\right\|_{\frac{3}{4}+\epsilon, \frac{7}{2}+3 \epsilon, 3} \leq(2 \pi \hbar)^{-\frac{1}{2}} \frac{\mu^{\prime}(\hbar)}{D_{\frac{9}{4}+\epsilon, 3}}:=(2 \pi \hbar)^{-\frac{1}{2}} \mu(\hbar)
$$

7.3.3. Hypothesis 2 in Theorem $2.1 \Longrightarrow$ Condition 2 in Theorem 2.2.

Since

$$
\begin{aligned}
W_{\hbar}\left[\left[x, \sqrt{R^{i n}}\right]\right](q, p) & =i \hbar \partial_{p} W_{\hbar}\left[\sqrt{R^{i n}}\right](q, p) \\
W_{\hbar}\left[\left[-i \hbar \nabla, \sqrt{R^{i n}}\right]\right](q, p) & =-i \hbar \partial_{q} W_{\hbar}\left[\sqrt{R^{i n}}\right](q, p),
\end{aligned}
$$

we get by Proposition 7.1, that the hypothesis (2) in Theorem 2.2 is satisfied as soon as

$$
\hbar\left\|\nabla W_{\hbar}\left[\sqrt{R^{i n}}\right]\right\|_{\frac{3}{4}+\epsilon, \frac{5}{2}+3 \epsilon, 3} \leq(2 \pi \hbar)^{-\frac{1}{2}} \frac{\nu^{\prime}(\hbar)}{D_{\frac{9}{4}+\epsilon, 3}}
$$

It is easy to check that, by the definition (15) of $\|a\|_{M, K, N}$

$$
\|\nabla a\|_{M, K, N} \leq \hbar^{-\frac{1}{2}} 2^{K}\|a\|_{M, K, N+1} .
$$

Indeed, let us do the computation in the $z, \bar{z}$ variables. We have

$$
\left|\left(\partial_{z} a\right)_{k}\right|=\left|\partial_{z} a_{k+1}\right|
$$


so that, since $|k-1|+1 \leq|k|+2=2(|k| / 2+1) \leq 2(|k|+1)$,

$$
\begin{aligned}
\left\|\partial_{z} a\right\|_{M, K, N} & =\sup _{z \in \mathbf{R}^{2 d}}\left(|z|^{2}+1\right)^{M}(|k|+1)^{K} \sup _{0 \leq|\alpha| \leq N}\left|(\sqrt{\hbar} D)^{\alpha} \partial_{z} a_{k(z)+1}\right| \\
& \leq \sup _{z \in \mathbf{R}^{2 d}}\left(|z|^{2}+1\right)^{M}(|k-1|+1)^{K} \sup _{0 \leq|\alpha| \leq N}\left|(\sqrt{\hbar} D)^{\alpha} \partial_{z} a_{k}(z)\right| \\
& \leq 2^{K} \hbar^{-\frac{1}{2}} \sup _{z \in \mathbf{R}^{2 d}}\left(|z|^{2}+1\right)^{M}(|k|+1)^{K} \sup _{0 \leq|\alpha| \leq N+1}\left|(\sqrt{\hbar} D)^{\alpha} a_{k}(z)\right| .
\end{aligned}
$$

The term $\partial_{\bar{z}} a$ is obtained the same way.

Therefore (86) is satisfied as soon as

$$
\hbar^{\frac{1}{2}}\left\|W_{\hbar}\left[\sqrt{R^{i n}}\right]\right\|_{\frac{3}{4}+\epsilon, \frac{5}{2}+3 \epsilon, 4} \leq(2 \pi \hbar)^{-\frac{1}{2}} \frac{\nu^{\prime}(\hbar)}{2^{\frac{5}{2}+3 \epsilon} D_{\frac{9}{4}+\epsilon, 4}}:=(2 \pi \hbar)^{-\frac{1}{2}} \nu(\hbar)
$$

7.4. The case of any dimension $d$. Let us perform the change of variable

$$
z_{l} \rightarrow\left(\left(j_{l}+\frac{1}{2}\right) \hbar\right)^{\frac{1}{2}} z_{l}, l=1, \ldots, d,
$$

and decompose $a=\sum a^{ \pm \bullet_{1}, \ldots, \pm \bullet_{d}}, \bullet_{l} \in\{\leq,=, \geq\}, l=1, \ldots, d$, where

$$
a_{j-j^{\prime}}^{ \pm \bullet_{1}, \ldots, \pm \bullet_{d}}\left(\frac{\sqrt{\left.\left(j+\frac{1}{2}\right)\right) \hbar} z}{\sqrt{2}}\right):=\prod_{l=1}^{d} \chi^{\bullet_{l}}\left(z_{l}\right) \chi_{\mathbf{N}}\left( \pm\left(\left(j_{l}-j_{l}^{\prime}\right)^{2}-j_{l}\right) a_{j-j^{\prime}}\left(\frac{\sqrt{\left.\left(j+\frac{1}{2}\right)\right) \hbar} z}{\sqrt{2}}\right),\right.
$$

where $\chi^{\bullet}$ is defined in $(74), \chi_{\mathbf{N}}$ is the characteristic function on $\mathbf{N}$ and, by abuse of notation,

$\left\langle j\left|\operatorname{Wig}\left(a_{j-j^{\prime}}^{ \pm \bullet_{1}, \ldots, \pm \bullet_{d}}\right)\right| j^{\prime}\right\rangle$ will involve $d$ integrations in the variable $z_{1}, \ldots, z_{d}$.

(1) For each $l$ such that the sign of $\bullet_{l}$ is positive, we will have $\left(j_{l}-j_{l}^{\prime}\right)^{2} \geq j_{l}$ so that the argument of the case (1) in the proceeding section apply (after a inverse change of variable $z_{l} \rightarrow\left(\left(j_{l}+\frac{1}{2}\right) \hbar\right)^{-\frac{1}{2}} z_{l}$.

(2) For each of the other variables $z_{k}$, the integral will be reduced to the domain of $\chi^{{ }^{*}}$ and can be treated as in the cases (a), (b), (c) of the preceding section.

(3) Combining in each dimension the different points (1), (2) (a), (2) (b) and (2) (c) of the proof of Proposition 7.1 in Section 7.3.1, we realize easily that (69) becomes in dimension $d$

$\mid\left\langle j^{\prime}\left|\operatorname{Wig}\left(a_{j-j^{\prime}}|j\rangle \mid \leq\left(D_{\mu, n} \hbar\right)^{d} 2^{(M+K) d}\right.\right.\right.$

$\times\left\|a_{j-j^{\prime}}\right\|_{M, 1+\epsilon+\tau, n}|| j^{\prime}-j\left|+\frac{1}{2}\right|^{-\min (1+\alpha+\epsilon, 1+\epsilon+\tau)}\left(\left(j+\frac{1}{2}\right) \hbar+\frac{1}{2}\right)^{-\min \left(\frac{\tau-\alpha-1}{2}, \mu-\frac{3}{2}, M, \frac{n-1}{2}\right)}$.

Therefore, $\sqrt{R^{i n}}$ satisfies the hypothesis (1) in Theorem 2.2 as soon as

$$
\left\|W_{\hbar}\left[\sqrt{R^{i n}}\right]\right\|_{\frac{3}{4}+\epsilon, \frac{7}{2}+3 \epsilon, 3} \leq(2 \pi \hbar)^{-\frac{d}{2}} \frac{\mu^{\prime}(\hbar)}{D_{\frac{9}{4}+\epsilon, 3}}=(2 \pi \hbar)^{-\frac{d}{2}} \mu(\hbar)
$$


By the same argument, hypothesis (2) in Theorem 2.2 is satisfied as soon as

$$
\hbar^{\frac{1}{2}}\left\|W_{\hbar}\left[\sqrt{R^{i n}}\right]\right\|_{\frac{3}{4}+\epsilon, \frac{5}{2}+3 \epsilon, 4} \leq(2 \pi \hbar)^{-\frac{d}{2}} \frac{\nu^{\prime}(\hbar)}{2^{\frac{5}{2}+3 \epsilon} D_{\frac{9}{4}+\epsilon, 4}}=(2 \pi \hbar)^{-\frac{d}{2}} \nu(\hbar)
$$

\subsection{End of the proof of item (I) in Theorem 4.1.}

We just proved that hypothesis (1) and (2) in Theorem 2.1 imply conditions(62) and (63) in Theorem 2.2 for

$$
\mu^{\prime}(\hbar)=D_{\frac{9}{4}+\epsilon, 3} \mu(\hbar), \nu^{\prime}(\hbar)=D_{\frac{9}{4}+\epsilon, 4} \nu(\hbar) .
$$

Therefore, by item $(I I)$, item $(I)$ holds true for

$$
D_{I}=D_{\frac{9}{4}+\epsilon, 4} D_{I I}
$$

since $D_{\frac{9}{4}+\epsilon, 4}>D_{\frac{9}{4}+\epsilon, 3}>1$.

\section{ThE CASE OF $N$ PARTICLES}

As it was already mentioned, a peculiar feature of Theorem 3.6 compared to usual semiclassical/microlocal methods is the fact that it provides an upper bound of a quantity at time $t$ linear in the same quantity at time $t=0$, without any extra remainder term to estimate. We will use this fact in the case of an $N$-body problem with factorized initial data through the use of the following result.

Lemma 8.1. Let $R_{1}, \ldots, R_{N}$ be $N$ density matrices on $L^{2}\left(\mathbf{R}^{d}\right)$ and let $f_{1}, \ldots, f_{N}$ be $N$ probability densities on $\mathbf{R}^{2 d}$. Let us denote

$$
\begin{aligned}
R & =R_{1} \otimes \cdots \otimes R_{N} \\
f(X, \Xi) & =f_{1}\left(x_{1}, \xi_{1}\right) \ldots f\left(x_{N}, \xi_{n}\right), X=\left(x_{1}, \ldots, x_{N}\right), \chi=\left(\xi_{1} \ldots \xi_{n}\right) .
\end{aligned}
$$

Then

$$
E_{\hbar}(f, R)^{2} \leq E_{\hbar}\left(f_{1}, R_{1}\right)^{2}+\cdots+E_{\hbar}\left(f_{N}, R_{N}\right)^{2} .
$$

Proof. Let

$$
\Pi(X, \Xi)=\pi_{1}^{o p}\left(x_{1}, \xi_{1}\right) \otimes \cdots \otimes \pi_{N}^{o p}\left(x_{N}, \xi_{N}\right),
$$

where $\pi_{i}^{o p}$ is an optimal coupling of $f_{i}$ and $R_{i}$. Then it is easy to see that $\Pi$ is a coupling of $f$ and $R$ and therefore, writing $c_{N}(X, \Xi)=\sum_{i=1}^{N} c_{i}\left(x_{i}, \xi_{i}\right)$ where $c_{i}\left(x_{i}, \xi_{i}\right)=$ $\frac{1}{2}\left(\left|x_{i}-y_{i}\right|^{2}+\left|\xi_{i}+i \hbar \nabla_{y_{i}}\right|^{2}\right)$ on $L^{2}\left(\mathbf{R}^{N d}, d y_{1} \ldots d y_{N}\right)$,

$$
\begin{aligned}
E_{\hbar}(f, R)^{2} & \leq \int \operatorname{trace} c_{N}(X, \Xi) \Pi(X, \Xi) d X d \Xi \\
& =\sum_{i=1}^{N} \int \operatorname{trace}\left(c_{i}\left(x_{i}, \xi_{i}\right) \pi_{i}^{o p}\left(x_{i}, \xi_{i}\right)\right) d x_{i} d \xi_{i} \leq \sum_{i=1}^{N} E_{\hbar}\left(f_{i}, R_{i}\right)^{2} .
\end{aligned}
$$


Lemma 8.1 suggests to multiply $E_{\hbar}(f, R)^{2}$ by $\frac{1}{N}$ in the situation involving $N$ particles. Likewise we will divide $\operatorname{dist}_{\mathrm{MK}, 2}^{2}$ by $N$ as is customary, and the square of the distance $\delta$ as well.

We will consider, as in [12], Section 2.3, the quantum evolution of interacting $N$ particles through the following von Neumann equation.

$$
i \hbar \frac{d}{d t} R_{N}(t)=\left[-\frac{1}{2} \hbar^{2} \Delta_{N}+V_{N}, R_{N}(t)\right], R_{N}(0)=R_{N}^{i n}
$$

with the factorized initial condition

$$
R_{N}^{i n}=\left(R^{i n}\right)^{\otimes N}
$$

where $R^{i n}$ is a density matrix on $L^{2}\left(\mathbf{R}^{d}\right)$.

In $(92), \Delta_{N}$ is the Laplacien on $L^{2}\left(\mathbf{R}^{N d}\right)$ and

$$
V_{N}\left(x_{1}, \ldots, x_{N}\right)=\frac{1}{N} \sum_{i<j} V\left(x_{1}-x_{j}\right), \quad V \in C^{1,1}\left(\mathbf{R}^{d}\right), \quad V \text { even, } V(0)=0 .
$$

Under these conditions on $V$, the $N$-body dynamics is well defined (see [12], Section $2.3)$.

We denote by $\rho_{N}(t)$ the solution of the corresponding Liouville equation on $\mathbf{R}^{2 d}$ with initial condition $\widetilde{W}\left[R_{N}^{i n}\right]=\left(W\left[R^{i n}\right]\right)^{\otimes N}$ :

$$
\dot{\rho}_{N}=\left\{\frac{1}{2} \sum_{i=1}^{N} \xi_{i}^{2}+\frac{1}{N} \sum_{i<j} V\left(x_{i}-x_{j}\right), \rho_{N}\right\}, \quad \rho(0)=\widetilde{W}\left[R^{i n}\right],
$$

and $\Phi_{N}^{t}$ the Hamiltonian flow of Hamiltonian $\frac{1}{2} \sum_{i=1}^{N} p_{i}^{2}+\frac{1}{N} \sum_{i<j} V\left(q_{i}-q_{j}\right)$ so that

$$
\rho_{N}(t)=\rho(0) \circ \Phi_{N}^{-t}
$$

Moreover we define again

$$
\lambda=\frac{1+\max \left(4 \operatorname{Lip}(\nabla V)^{2}, 1\right)}{2} .
$$

Theorem 8.2. Let either $W_{\hbar}\left[\sqrt{R^{i n}}\right]$ satisfy the hypothesis of Theorem 2.1, or $\sqrt{R^{i n}}$ satisfy the hypothesis of Theorem 2.2 or Theorem 2.3. Let us suppose for simplicity that $\mu(\hbar)=\mu^{\prime}(\hbar)=1, \nu(\hbar)=\nu^{\prime}(\hbar)=\hbar$ (the general case is straightforward to state). Then

$$
\frac{1}{N} \operatorname{dist}_{\mathrm{MK}, 2}\left(\widetilde{W}_{\hbar}\left[R_{N}(t)\right], \widetilde{W}_{\hbar}\left[R_{N}^{i n}\right] \circ \Phi_{N}^{-t}\right)^{2} \leq C e^{\lambda|t|} \hbar
$$

where $C$ is independent of $N$.

Moreover, if $\left\|W_{\hbar}\left[R^{i n}\right]\right\|_{L^{2}\left(\mathbf{R}^{2 d}\right)} \leq C^{\prime}<1$, uniformly in $\hbar$, that is to say $\left\|R^{\text {in }}\right\|_{2} \leq$ $C^{\prime}(2 \pi \hbar)^{d}$, then

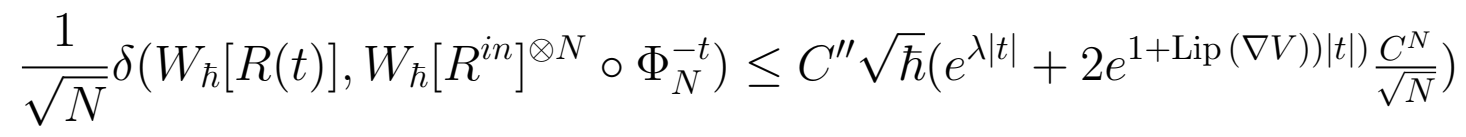


where $C^{\prime \prime}$ is independent of $N$.

Proof. By Theorem 2.7 in [12] in the case $n=N$ we know that, by Lemma 8.1,

$$
\frac{1}{N} E\left(\widetilde{W}_{\hbar}\left[R_{N}^{i n} \circ \Phi_{N}(t)\right], R_{N}(t)\right)^{2} \leq e^{\lambda|t|} \frac{1}{N} E\left(\widetilde{W}_{\hbar}\left[R_{N}^{i n}\right], R_{N}^{i n}\right)^{2} \leq e^{\lambda|t|} E\left(\widetilde{W}_{\hbar}\left[R^{i n}\right], R^{i n}\right)^{2} .
$$

Theorem 3.3, (3) and Theorem 4.1, I - III, give then the first inequality of Theorem 8.2 .

Theorem B.4 gives directly the second one.

\section{Appendix A. Proof of the triangle inequality}

In this section, we prove Theorem 3.5.

The proof makes use of some inequalities between the (classical and/or quantum) transportation cost operators. We begin with an elementary, but useful lemma, which can be viewed as the Peter-Paul inequality for operators.

Lemma A.1. Let $T, S$ be unbounded self-adjoint operators on $\mathfrak{H}=L^{2}\left(\mathbf{R}^{n}\right)$, with domains $\operatorname{Dom}(T)$ and $\operatorname{Dom}(S)$ respectively such that $\operatorname{Dom}(T) \cap \operatorname{Dom}(S)$ is dense in $\mathfrak{H}$. Then, for all $\alpha>0$, one has

$$
\langle v|T S+S T| v\rangle \leq \alpha\left\langle v\left|T^{2}\right| v\right\rangle+\frac{1}{\alpha}\left\langle v\left|S^{2}\right| v\right\rangle, \quad \text { for all } v \in \operatorname{Dom}(T) \cap \operatorname{Dom}(S) .
$$

Proof. Indeed, for each $\alpha>0$ and each $v \in \operatorname{Dom}(T) \cap \operatorname{Dom}(S)$, one has

$$
\begin{array}{r}
\alpha\left\langle v\left|T^{2}\right| v\right\rangle+\frac{1}{\alpha}\left\langle v\left|S^{2}\right| v\right\rangle-\langle v|T S+S T| v\rangle \\
=|\sqrt{\alpha} T v|^{2}+\left|\frac{1}{\sqrt{\alpha}} S v\right|^{2}-\left\langle\sqrt{\alpha} T v \mid \frac{1}{\sqrt{\alpha}} S v\right\rangle-\left\langle\frac{1}{\sqrt{\alpha}} S v \mid \sqrt{\alpha} T v\right\rangle \\
=\left|\sqrt{\alpha} T v-\frac{1}{\sqrt{\alpha}} S v\right|^{2} \geq 0 .
\end{array}
$$

Let us redefine the $\operatorname{cost} c_{\hbar}(x, \xi)$ that way

$$
c\left(x, \xi ; z, \hbar D_{z}\right):=|x-z|^{2}+\left|\xi-\hbar D_{z}\right|^{2} .
$$

Lemma A.2. For each $x, \xi, y, \eta, z \in \mathbf{R}^{d}$ and each $\alpha>0$, one has

$$
\begin{gathered}
c\left(x, \xi ; z, \hbar D_{z}\right) \leq(1+\alpha)\left(|x-y|^{2}+|\xi-\eta|^{2}\right)+\left(1+\frac{1}{\alpha}\right) c\left(y, \eta ; z, \hbar D_{z}\right), \\
|x-z|^{2}+|\xi-\zeta|^{2} \leq(1+\alpha) c\left(x, \xi ; y, \hbar D_{y}\right)+(1+\alpha) c\left(z, \zeta ; y, \hbar D_{y}\right) .
\end{gathered}
$$

These two inequalities are of the form $A \leq B$ where $A$ and $B$ are unbounded selfadjoint operators on $L^{2}\left(\mathbf{R}^{n}\right)$ for some $n \geq 1$, with

$$
\mathcal{W}:=\left\{\psi \in H^{1}\left(\mathbf{R}^{n}\right) \text { s.t. }|x| \psi \in \mathcal{H}\right\} \subset \operatorname{Dom}_{f}(A) \cap \operatorname{Dom}_{f}(B),
$$

denoting by $\operatorname{Dom}_{f}(A)$ (resp. $\left.\operatorname{Dom}_{f}(B)\right)$ the form-domain of $A$ (resp. of $B$ ) - see $\S$ VIII.6 in [17] on pp. 276-277. The inequality $A \leq B$ means that the bilinear form associated to $B-A$ is nonnegative, i.e. that

$$
\langle w|A| w\rangle \leq\langle w|B| w\rangle, \quad \text { for all } w \in \mathcal{W} .
$$


Proof. These two inequalities are proved in the same way. Let us prove for instance the first inequality:

$$
\begin{aligned}
c\left(x, \xi ; z, \hbar D_{z}\right)= & |x-y+y-z|^{2}+\left|\xi-\eta+\eta-\hbar D_{z}\right|^{2} \\
= & |x-y|^{2}+|\xi-\eta|^{2}+c\left(y, \eta ; z, \hbar D_{z}\right) \\
& +2(x-y) \cdot(y-z)+2(\xi-\eta) \cdot\left(\eta-\hbar D_{z}\right) .
\end{aligned}
$$

By Lemma A.1 with $T=(x-y) \mathbb{I}_{z}$ and $S=\eta-\hbar D_{z}$ we get

$$
\begin{aligned}
& 2(x-y) \cdot(y-z)+2(\xi-\eta) \cdot\left(\eta-\hbar D_{z}\right) \\
\leq & \alpha\left(|x-y|^{2}+|\xi-\eta|^{2}\right)+\frac{1}{\alpha} c\left(y, \eta ; z, \hbar D_{z}\right),
\end{aligned}
$$

which concludes the proof of the first inequality.

Finally we will need the following proposition whose proof is postponed to the end of this section.

Proposition A.3. Let $A=A^{*} \geq 0$ be an unbounded self-adjoint operator on $\mathcal{H}$ with domain $\operatorname{Dom}(A)$, and let $E$ be its spectral decomposition. Let $T \in \mathcal{L}^{1}(\mathfrak{H})$ satisfy $T=$ $T^{*} \geq 0$, and let $\left(e_{j}\right)_{j \geq 1}$ be a complete orthonormal system of eigenvectors of $T$ with $T e_{j}=\tau_{j} e_{j}$ and $\tau_{j} \in[0,+\infty)$ for each $j \geq 1$.

Assume that

$$
\sum_{j \geq 1} \tau_{j} \int_{0}^{\infty} \lambda\left\langle e_{j}|E(d \lambda)| e_{j}\right\rangle<\infty .
$$

Let $\Phi_{n}: \mathbf{R}_{+} \rightarrow \mathbf{R}_{+}$be a sequence of continuous, bounded and nondecreasing functions such that

Set

$$
0 \leq \Phi_{1}(r) \leq \Phi_{2}(r) \leq \ldots \leq \Phi_{n}(r) \rightarrow r \quad \text { as } n \rightarrow \infty
$$

$$
\Phi_{n}(A):=\int_{0}^{\infty} \Phi_{n}(\lambda) E(d \lambda) \in \mathcal{L}(\mathcal{H})
$$

Then $\Phi_{n}(A)=\Phi_{n}(A)^{*} \geq 0$ for each $n \geq 1$ and the sequence $T^{1 / 2} \Phi_{n}(A) T^{1 / 2}$ converges weakly to $T^{1 / 2} A T^{1 / 2}$ as $n \rightarrow \infty$. Moreover

$$
\operatorname{trace}_{\mathcal{H}}\left(T \Phi_{n}(A)\right) \rightarrow \operatorname{trace}_{\mathcal{H}}\left(T^{1 / 2} A T^{1 / 2}\right) \quad \text { as } n \rightarrow \infty .
$$

Proof of Theorem 3.5.

We start by the following "disintegration" result.

Lemma A.4. Let $f \in \mathcal{P}^{a c}\left(\mathbf{R}^{d} \times \mathbf{R}^{d}\right)$, let $R \in \mathcal{D}(\mathfrak{H})$ and let $Q \in \mathcal{C}(f, R)$. There exists a $\sigma\left(\mathcal{L}^{1}(\mathfrak{H}), \mathcal{L}(\mathfrak{H})\right)$ weakly measurable function $(x, \xi) \mapsto Q_{f}(x, \xi)$ defined a.e. on $\mathbf{R}^{d} \times \mathbf{R}^{d}$ with values in $\mathcal{L}^{1}(\mathfrak{H})$ such that

$$
Q_{f}(x, \xi)=Q_{f}^{*}(x, \xi) \geq 0, \quad \operatorname{trace}\left(Q_{f}(x, \xi)\right)=1, \quad \text { and } Q(x, \xi)=f(x, \xi) Q_{f}(x, \xi)
$$

for a.e. $(x, \xi) \in \mathbf{R}^{d} \times \mathbf{R}^{d}$. 
Proof of the Lemma.

Let $f_{1}$ be a Borel measurable function defined on $\mathbf{R}^{d} \times \mathbf{R}^{d}$ and such that $f(x, \xi)=$ $f_{1}(x, \xi)$ for a.e. $(x, \xi) \in \mathbf{R}^{d} \times \mathbf{R}^{d}$. Let $\mathcal{N}$ be the Borel measurable set defined as follows: $\mathcal{N}:=\left\{(x, \xi) \in \mathbf{R}^{d} \times \mathbf{R}^{d}\right.$ s.t. $\left.f(x, \xi)=0\right\}$, and let $u \in \mathfrak{H}$ satisfy $|u|=1$. Consider the function

$$
(x, \xi) \mapsto Q_{f}(x, \xi):=\frac{Q(x, \xi)+\mathbf{1}_{\mathcal{N}}(x, \xi)|u\rangle\langle u|}{f_{1}(x, \xi)+\mathbf{1}_{\mathcal{N}}(x, \xi)} \in \mathcal{L}(\mathfrak{H})
$$

defined a.e. on $\mathbf{R}^{d} \times \mathbf{R}^{d}$. The function $f_{1}+\mathbf{1}_{\mathcal{N}}>0$ is Borel measurable on $\mathbf{R}^{d} \times \mathbf{R}^{d}$ while $(x, \xi) \mapsto\langle\phi|Q(x, \xi)| \psi\rangle$ is measurable and defined a.e. on $\mathbf{R}^{d} \times \mathbf{R}^{d}$ for each $\phi, \psi \in \mathfrak{H}$. Set $\mathcal{A}: \mathcal{L}(\mathfrak{H}) \times(0,+\infty) \ni(T, \lambda) \mapsto \lambda^{-1} T \in \mathcal{L}(\mathfrak{H}) ;$ since $\mathcal{A}$ is continuous, the function $Q_{f}:=\mathcal{A}\left(Q+\mathbf{1}_{\mathcal{N}} \otimes|u\rangle\langle u|, f_{1}+\mathbf{1}_{\mathcal{N}}\right)$ is weakly measurable on $\mathbf{R}^{d} \times \mathbf{R}^{d}$. Since $f_{1}+\mathbf{1}_{\mathcal{N}}>0$, and since $Q(x, \xi)=Q^{*}(x, \xi) \geq 0$, one has $\left(Q(x, \xi)+\mathbf{1}_{\mathcal{N}} \otimes|u\rangle\langle u|\right)^{*}=$ $Q(x, \xi)+\mathbf{1}_{\mathcal{N}} \otimes|u\rangle\langle u| \geq 0$ for a.e. $(x, \xi) \in \mathbf{R}^{d} \times \mathbf{R}^{d}$. On the other hand, for a.e. $(x, \xi) \in \mathbf{R}^{d} \times \mathbf{R}^{d}$, one has $\operatorname{trace}\left(Q(x, \xi)+\mathbf{1}_{\mathcal{N}} \otimes|u\rangle\langle u|\right)=f(x, \xi)+\mathbf{1}_{\mathcal{N}}(x, \xi)$, so that $\operatorname{trace}\left(Q_{f}(x, \xi)\right)=1$. Finally

$$
f(x, \xi) Q_{f}(x, \xi)=\frac{f(x, \xi) Q(x, \xi)}{f_{1}(x, \xi)+\mathbf{1}_{\mathcal{N}}(x, \xi)}=Q(x, \xi) \quad \text { for a.e. }(x, \xi) \in \mathbf{R}^{d} \times \mathbf{R}^{d},
$$

since $f=f_{1}$ a.e. on $\mathbf{R}^{d} \times \mathbf{R}^{d}$ and $\mathbf{1}_{\mathcal{N}}(x, \xi)=0$ for a.e. $(x, \xi) \in \mathbf{R}^{d} \times \mathbf{R}^{d}$ such that $f(x, \xi)>0$. Since $Q_{f}$ satisfies trace $\left(Q_{f}(x, \xi)\right)=1$ for a.e. $(x, \xi) \in \mathbf{R}^{d} \times \mathbf{R}^{d}$ and is weakly measurable on $\mathbf{R}^{d} \times \mathbf{R}^{d}$, it is $\sigma\left(\mathcal{L}^{1}(\mathfrak{H}), \mathcal{L}(\mathfrak{H})\right)$ weakly measurable.

By Theorem 2.12 in chapter 2 of [19], there exists an optimal coupling for $W_{2}(f, g)$, of the form $f(x, \xi) \delta_{\nabla \Phi(x, \xi)}(d y d \eta)$, where $\Phi$ is a convex function on $\mathbf{R}^{d} \times \mathbf{R}^{d}$. Let $Q \in \mathcal{C}\left(g, R_{1}\right)$ and set

$$
P(x, \xi ; d y d \eta):=f(x, \xi) \delta_{\nabla \Phi(x, \xi)}(d y d \eta) Q_{g}(y, \eta),
$$

where $Q_{g}$ is the disintegration of $Q$ with respect to $f$ obtained in Lemma A.4. Then $P$ is a nonnegative, self-adjoint operator-valued measure satisfying

$$
\operatorname{trace}_{\mathfrak{H}}(P(x, \xi ; d y d \eta))=f(x, \xi) \delta_{\nabla \Phi(x, \xi)}(d y d \eta)
$$

while

$$
\int P d x d \xi=(\nabla \Phi \# f)(y, \eta) d y d \eta Q_{g}(y, \eta)=g(y, \eta) Q_{g}(y, \eta) d y d \eta=Q(y, \eta) d y d \eta .
$$

In particular

$$
\int P(x, \xi ; d y d \eta)=f(x, \xi) Q_{g}(\nabla \Phi(x, \xi)) \in \mathcal{C}\left(f, R_{1}\right)
$$

Therefore

$$
E_{\hbar}\left(f, R_{1}\right)^{2} \leq \int \operatorname{trace}_{\mathfrak{H}}\left(Q_{g}(\nabla \Phi(x, \xi))^{1 / 2} c_{\hbar}(x, \xi) Q_{g}(\nabla \Phi(x, \xi))^{1 / 2}\right) f(x, \xi) d x d \xi .
$$

By the first inequality in Lemma A.2, one has

$$
c\left(x, \xi ; z, \hbar D_{z}\right) \leq(1+\alpha)|(x, \xi)-\nabla \phi(x, \xi)|^{2}+\left(1+\frac{1}{\alpha}\right) c\left(\nabla \Phi(x, \xi) ; z, \hbar D_{z}\right)
$$


for a.e. $(x, \xi) \in \mathbf{R}^{d} \times \mathbf{R}^{d}$ and all $\alpha>0$. Since $g \in \mathcal{P}_{2}^{a c}\left(\mathbf{R}^{d} \times \mathbf{R}^{d}\right)$ and $R_{1} \in \mathcal{D}_{2}(\mathfrak{H})$ and $Q \in \mathcal{C}\left(g, R_{1}\right)$, then

$$
\begin{gathered}
\int \operatorname{trace}_{\mathfrak{H}}\left(Q(y, \eta)^{1 / 2} c(y, \eta) Q(y, \eta)^{1 / 2}\right) d y d \eta \\
=\int \operatorname{trace}_{\mathfrak{H}}\left(Q_{g}(\nabla \Phi(x, \xi))^{1 / 2} c(\nabla \Phi(x, \xi)) Q_{g}(\nabla \Phi(x, \xi))^{1 / 2}\right) f(x, \xi) d x d \xi<\infty .
\end{gathered}
$$

For each $\epsilon>0$, set

$$
c^{\epsilon}\left(x, \xi ; z, \hbar D_{z}\right)=\left(I+\epsilon c\left(x, \xi ; z, \hbar D_{z}\right)\right)^{-1} c\left(x, \xi ; z, \hbar D_{z}\right) \leq c\left(x, \xi ; z, \hbar D_{z}\right) .
$$

Then, for a.e. $(x, \xi) \in \mathbf{R}^{d} \times \mathbf{R}^{d}$ and each $\epsilon>0$, one has

$$
Q_{g}(\nabla \Phi(x, \xi))^{1 / 2} c\left(\nabla \Phi(x, \xi) ; z, \hbar D_{z}\right) Q_{g}(\nabla \Phi(x, \xi))^{1 / 2} \in \mathcal{L}^{1}(\mathfrak{H})
$$

and

$$
\begin{aligned}
Q_{g}(\nabla \Phi(x, \xi))^{1 / 2} & c_{\hbar}^{\epsilon}\left(x, \xi ; z, \hbar D_{z}\right) Q_{g}(\nabla \Phi(x, \xi))^{1 / 2} \\
\leq & (1+\alpha)|(x, \xi)-\nabla \phi(x, \xi)|^{2} Q_{g}(\nabla \Phi(x, \xi)) \\
& +\left(1+\frac{1}{\alpha}\right) Q_{g}(\nabla \Phi(x, \xi))^{1 / 2} c\left(\nabla \Phi(x, \xi) ; z, \hbar D_{z}\right) Q_{g}(\nabla \Phi(x, \xi))^{1 / 2}
\end{aligned}
$$

Integrating both sides of this inequality with respect to the probability distribution $f(x, \xi)$, one finds

$$
\begin{array}{r}
\int \operatorname{trace}_{\mathfrak{H}}\left(Q_{g}(\nabla \Phi(x, \xi))^{1 / 2} c_{\hbar}^{\epsilon}(x, \xi) Q_{g}(\nabla \Phi(x, \xi))^{1 / 2}\right) f(x, \xi) d x d \xi \\
\leq(1+\alpha) \int|(x, \xi)-\nabla \phi(x, \xi)|^{2} f(x, \xi) d x d \xi \\
+\left(1+\frac{1}{\alpha}\right) \int \operatorname{trace}_{\mathfrak{H}}\left(Q_{g}(\nabla \Phi(x, \xi))^{1 / 2} c(\nabla \Phi(x, \xi)) Q_{g}(\nabla \Phi(x, \xi))^{1 / 2}\right) f(x, \xi) d x d \xi \\
\leq(1+\alpha) \operatorname{dist}_{\mathrm{MK}, 2}(f, g)^{2} \\
+\left(1+\frac{1}{\alpha}\right) \int \operatorname{trace}_{\mathfrak{H}}\left(Q_{g}(y, \eta)^{1 / 2} c(y, \eta) Q_{g}(y, \eta)^{1 / 2}\right) g(y, \eta) d y d \eta \\
\leq(1+\alpha) \operatorname{dist}_{\mathrm{MK}, 2}(f, g)^{2}+\left(1+\frac{1}{\alpha}\right) \int \operatorname{trace}_{\mathfrak{H}}\left(Q(y, \eta)^{1 / 2} c(y, \eta) Q(y, \eta)^{1 / 2}\right) d y d \eta
\end{array}
$$

Minimizing the last right hand side of this inequality in $Q \in \mathcal{C}\left(g, R_{1}\right)$ shows that

$$
\begin{array}{r}
\int \operatorname{trace}_{\mathfrak{H}}\left(Q_{g}(\nabla \Phi(x, \xi))^{1 / 2} c_{\hbar}^{\epsilon}(x, \xi) Q_{g}(\nabla \Phi(x, \xi))^{1 / 2}\right) f(x, \xi) d x d \xi \\
\leq(1+\alpha) \operatorname{dist}_{\mathrm{MK}, 2}(f, g)^{2}+\left(1+\frac{1}{\alpha}\right) E\left(g, R_{1}\right)^{2}
\end{array}
$$

Passing to the limit as $\epsilon \rightarrow 0^{+}$in the left hand side and applying Proposition A.3 shows that

$$
\begin{aligned}
E_{\hbar}\left(f, R_{1}\right)^{2} & \leq \int \operatorname{trace}_{\mathfrak{H}}\left(Q_{g}(\nabla \Phi(x, \xi))^{1 / 2} c(x, \xi) Q_{g}(\nabla \Phi(x, \xi))^{1 / 2}\right) f(x, \xi) d x d \xi \\
& \leq(1+\alpha) \operatorname{dist}_{\mathrm{MK}, 2}(f, g)^{2}+\left(1+\frac{1}{\alpha}\right) E\left(g, R_{1}\right)^{2},
\end{aligned}
$$

the first inequality being a consequence of the definition of $E_{\hbar}$ according to (99). 
Finally, minimizing the right hand side of this inequality as $\alpha>0$, i.e. choosing $\alpha=E_{\hbar}(f, g) / \operatorname{dist}_{\mathrm{MK}, 2}(f, g)$ if $f \neq g$ a.e. on $\mathbf{R}^{d} \times \mathbf{R}^{d}$, or letting $\alpha \rightarrow+\infty$ if $f=g$, we arrive at the inequality

$$
\begin{aligned}
E_{\hbar}\left(f, R_{1}\right)^{2} & \leq \operatorname{dist}_{\mathrm{MK}, 2}(f, g)^{2}+E_{\hbar}\left(g, R_{1}\right)^{2}+2 E_{\hbar}\left(g, R_{1}\right) \operatorname{dist}_{\mathrm{MK}, 2}(f, g) \\
& =\left(\operatorname{dist}_{\mathrm{MK}, 2}(f, g)+E_{\hbar}\left(g, R_{1}\right)\right)^{2}
\end{aligned}
$$

which is precisely the desired inequality. Theorem 3.5 is proven.

Proof of Proposition A.3. Since $E$ is a resolution of the identity on $[0,+\infty)$, and since $\Phi_{n}$ is continuous, bounded and with values in $[0,+\infty)$, the operators $\Phi_{n}(A)$ satisfy

$$
0 \leq \Phi_{n}(A)=\Phi_{n}(A)^{*} \leq\left(\sup _{z \geq 0} \Phi_{n}(z)\right) I_{\mathcal{H}}
$$

and

$$
0 \leq \Phi_{1}(A) \leq \Phi_{2}(A) \leq \ldots \leq \Phi_{n}(A) \leq \ldots
$$

Set $R_{n}:=T^{1 / 2} \Phi_{n}(A) T^{1 / 2}$; by definition $0 \leq R_{n}=R_{n}^{*} \in \mathcal{L}^{1}(\mathfrak{H})$ and one has

$$
0 \leq R_{1} \leq R_{2} \leq \ldots \leq R_{n} \leq \ldots
$$

together with

$$
\operatorname{trace}_{\mathcal{H}}\left(R_{n}\right)=\sum_{j \geq 1} \tau_{j} \int_{0}^{\infty} \Phi_{n}(\lambda)\left\langle e_{j}|E(d \lambda)| e_{j}\right\rangle \leq \sum_{j \geq 1} \tau_{j} \int_{0}^{\infty} \lambda\left\langle e_{j}|E(d \lambda)| e_{j}\right\rangle<\infty
$$

by (98).

Therefore, since $0 \leq R_{n} \in \mathcal{L}^{1}(\mathcal{H})$, $\sup _{n \geq 1}\left\langle x\left|R_{n}\right| x\right\rangle \leq \sup _{n \geq 1} \||x\rangle\langle x|\left\|_{\mathcal{L}(\mathcal{H})} \operatorname{trace}{ }_{\mathcal{H}}\left(R_{n}\right)=\right\| x \|_{\mathcal{H}}^{2} \sup _{n \geq 1} \operatorname{trace} \mathcal{H}_{\mathcal{H}}\left(R_{n}\right)<\infty$ for all $x \in \mathcal{H}$. Since the sequence $\left\langle x\left|R_{n}\right| x\right\rangle \in[0,+\infty)$ is nondecreasing for each $x \in \mathcal{H}$,

$$
\left\langle x\left|R_{n}\right| x\right\rangle \rightarrow \sup _{n \geq 1}\left\langle x\left|R_{n}\right| x\right\rangle=: q(x) \in[0,+\infty) \quad \text { for all } x \in \mathcal{H}
$$

as $n \rightarrow \infty$. Hence

$$
\left\langle x\left|R_{n}\right| y\right\rangle=\left\langle y\left|R_{n}\right| x\right\rangle \rightarrow \frac{1}{4}(q(x+y)-q(x-y)+i q(x-i y)-i q(x+i y))=: b(x, y) \in \mathbf{C}
$$

as $n \rightarrow+\infty$. By construction, $b$ is a nonnegative sesquilinear form on $\mathcal{H}$.

Consider, for each $k \geq 0$,

$$
F_{k}:=\left\{x \in \mathcal{H} \text { s.t. }\left\langle x\left|R_{n}\right| x\right\rangle \leq k \text { for each } n \geq 1\right\} .
$$

The set $F_{k}$ is closed for each $k \geq 0$, being the intersection of the closed sets defined by the inequality $\left\langle x\left|R_{n}\right| x\right\rangle \leq k$ as $n \geq 1$. Since the sequence $\left\langle x\left|R_{n}\right| x\right\rangle$ is bounded for each $x \in \mathcal{H}$,

$$
\bigcup_{k \geq 0} F_{k}=\mathcal{H}
$$

Applying Baire's theorem shows that there exists $N \geq 0$ such that $\stackrel{\circ}{F}_{N} \neq \varnothing$. In other words, there exists $r>0$ and $x_{0} \in \mathcal{H}$ such that

$$
\left|x-x_{0}\right| \leq r \Longrightarrow\left|\left\langle x\left|R_{n}\right| x\right\rangle\right| \leq N \text { for all } n \geq 1 \text {. }
$$


By linearity and positivity of $R_{n}$, this implies

$$
\left|\left\langle z\left|R_{n}\right| z\right\rangle\right| \leq \frac{2}{r}(M+N)\|z\|^{2} \text { for all } n \geq 1, \quad \text { with } M:=\sup _{n \geq 1}\left\langle x_{0}\left|R_{n}\right| x_{0}\right\rangle .
$$

In particular

$$
\sup _{|z| \leq 1} q(z) \leq \frac{2}{r}(M+N), \quad \text { so that }|b(x, y)| \leq \frac{2}{r}(M+N) \mid\|x\|_{\mathcal{H}}\|y\|_{\mathcal{H}}
$$

for each $x, y \in \mathcal{H}$ by the Cauchy-Schwarz inequality. By the Riesz representation theorem, there exists $R \in \mathcal{L}(\mathcal{H})$ such that

$$
R=R^{*} \geq 0, \quad \text { and } \quad b(x, y)=\langle x|R| y\rangle=\lim _{n \rightarrow \infty}\left\langle x\left|R_{n}\right| y\right\rangle,
$$

so that $R_{n} \rightarrow R \in \mathcal{L}(\mathcal{H})$ weakly as $n \rightarrow \infty$.

Observe now that $R \geq R_{n}$ for each $n \geq 1$, so that

In particular

$$
\sup _{n \geq 1} \operatorname{trace}_{\mathcal{H}}\left(R_{n}\right) \leq \operatorname{trace}_{\mathcal{H}}(R) \text {. }
$$

$$
\sup _{n \geq 1} \operatorname{trace}_{\mathcal{H}}\left(R_{n}\right)=+\infty \Longrightarrow \operatorname{trace}_{\mathcal{H}}(R)=+\infty .
$$

Since the sequence $\operatorname{trace}_{\mathcal{H}}\left(R_{n}\right)$ is nondecreasing,

$$
\operatorname{trace}_{\mathcal{H}}\left(R_{n}\right) \rightarrow \sup _{n \geq 1} \operatorname{trace}_{\mathcal{H}}\left(R_{n}\right) \quad \text { as } n \rightarrow \infty \text {. }
$$

By the noncommutative variant of Fatou's lemma (Theorem 2.7 (d) in [18]),

$$
\sup _{n \geq 1} \operatorname{trace}_{\mathcal{H}}\left(R_{n}\right)<\infty \Longrightarrow R \in \mathcal{L}^{1}(\mathcal{H}) \text { and } \operatorname{trace}_{\mathcal{H}}(R) \leq \sup _{n \geq 1} \operatorname{trace}_{\mathcal{H}}\left(R_{n}\right)
$$

so that, by $(100)$,

$$
\operatorname{trace}_{\mathcal{H}}(R)=\sup _{n \geq 1} \operatorname{trace}_{\mathcal{H}}\left(R_{n}\right) .
$$

Finally

$$
T^{1 / 2} A T^{1 / 2}-R_{n}=\sum_{j, k \geq 1} \tau_{j}^{1 / 2} \tau_{k}^{1 / 2}\left(\int_{0}^{\infty}\left(\lambda-\Phi_{n}(\lambda)\right)\left\langle e_{j}|E(d \lambda)| e_{k}\right\rangle\right)\left|e_{j}\right\rangle\left\langle e_{k}\right|
$$

so that

$$
\begin{aligned}
\left\langle x\left|T^{1 / 2} A T^{1 / 2}-R_{n}\right| x\right\rangle & =\int_{0}^{\infty}\left(\lambda-\Phi_{n}(\lambda)\right)\left\langle\sum_{j \geq 1} \tau_{j}^{1 / 2}\left\langle e_{j} \mid x\right\rangle e_{j}|E(d \lambda)| \sum_{k \geq 1} \tau_{k}^{1 / 2}\left\langle e_{k} \mid x\right\rangle e_{k}\right\rangle \\
& =\int_{0}^{\infty}\left(\lambda-\Phi_{n}(\lambda)\right)\left\langle T^{1 / 2} x|E(d \lambda)| T^{1 / 2} x\right\rangle \geq 0 .
\end{aligned}
$$

Hence

$$
0 \leq T^{1 / 2} A T^{1 / 2}-R_{n}=\left(T^{1 / 2} A T^{1 / 2}-R_{n}\right)^{*} \in \mathcal{L}^{1}(\mathcal{H})
$$

so that

$$
\begin{aligned}
\left\|T^{1 / 2} A T^{1 / 2}-R_{n}\right\|_{1} & =\operatorname{trace} \mathcal{H}\left(T^{1 / 2} A T^{1 / 2}-R_{n}\right) \\
& =\sum_{j \geq 1} \tau_{j} \int_{0}^{\infty}\left(\lambda-\Phi_{n}(\lambda)\right)\left\langle e_{j}|E(d \lambda)| e_{j}\right\rangle \rightarrow 0
\end{aligned}
$$


as $n \rightarrow \infty$ by monotone convergence. Hence $R_{n} \rightarrow T^{1 / 2} A T^{1 / 2}$ in $\mathcal{L}^{1}(\mathcal{H})$ and one has in particular

$$
\operatorname{trace}_{\mathcal{H}}\left(T \Phi_{n}(A)\right)=\operatorname{trace}_{\mathcal{H}}\left(T^{1 / 2} \Phi_{n}(A) T^{1 / 2}\right) \rightarrow \operatorname{trace}_{\mathcal{H}}\left(T^{1 / 2} A T^{1 / 2}\right) .
$$

\section{Appendix B. Quantum Wasserstein and Weak topologies}

It is well known that Wasserstein metrics dominate weak topologies. It is natural to wonder whether the $E_{\hbar}$ does the same. The answer will be positive when considering, for two density matrices $S$ and $R$, the quantity $E_{\hbar}\left(\widetilde{W}_{\hbar}[S], R\right)$. Since, by Theorem $3.3,3)$,

$$
E_{\hbar}\left(\widetilde{W}_{\hbar}[S], R\right) \geq \frac{1}{\sqrt{2}} \operatorname{dist}_{\mathrm{MK}, 2}\left(\widetilde{W}_{\hbar}[S], \widetilde{W}_{\hbar}[R]\right),
$$

the following results will give the answer.

Notation: unless the contrary is specified, we will define the norm of a vector as the maximum value of the norm over all the components.

B.1. $L^{2}$ test functions. We will denote by $\mathcal{D}_{H S}(\mathfrak{H})$ the set of Hilbert-Schmidt operators on $\mathfrak{H}$.

Definition B.1. Let $R, S \in \mathcal{D}_{H S}(\mathfrak{H})$, with Wigner transforms $W_{\hbar}[R], W_{\hbar}[S]$. We define

$$
d(R, S)=\sup _{\|F\|_{1},\left\|\frac{1}{i \hbar}[x, F]\right\|_{1},\left\|\frac{1}{i \hbar}[-i \hbar \nabla, F]\right\|_{1} \leq 1}|\operatorname{trace}(F(R-S))|
$$

and

$$
\delta\left(W_{\hbar}[R], W_{\hbar}[S]\right)=\sup _{\substack{\operatorname{Lip}(f) \leq 1 \\\|f\|_{L^{2}\left(\mathbf{R}^{2 d}\right)},\|\nabla f\|_{L^{2}\left(\mathbf{R}^{2 d)} \leq 1\right.}}}\left|\int\left(W_{\hbar}[R]-W_{\hbar}[S]\right) f(x, \xi) d x d \xi\right|
$$

Note that, for $R, S \in \mathcal{D}_{H S}(\mathfrak{H})$, we have $d(R, S) \leq\|R-S\| \leq\|R-S\|_{H S}<\infty$ and $\delta\left(W_{\hbar}[R], W_{\hbar}[S)\right) \leq(2 \pi \hbar)^{-d / 2}\|R-S\|_{H S}<\infty$.

Proposition B.2. Let $\hbar \leq \frac{2}{\pi}$.

Then the functions $d(\cdot, \cdot), \delta\left(W_{\hbar}[\cdot], W_{\hbar}[\cdot]\right): \mathcal{D}_{H S}(\mathfrak{H}) \times \mathcal{D}_{H S}(\mathfrak{H}) \rightarrow[0,+\infty[$ are distances.

Moreover,

$$
d(\cdot, \cdot) \leq 2^{d} \delta\left(W_{\hbar}[\cdot], W_{\hbar}[\cdot]\right)
$$

Proof. We first prove the inequality. Let us recall the following elementary facts:

$$
\operatorname{trace}(F(R-S))=(2 \pi \hbar)^{d} \int W_{\hbar}[F]\left(W_{\hbar}[R]-W_{\hbar}[S]\right)(x, \xi) d x d \xi
$$

and $W_{\hbar}\left[\frac{1}{i \hbar}[x, F]\right]=\nabla_{p} W_{\hbar}[F], W_{\hbar}\left[\frac{1}{i \hbar}[-i \hbar \nabla, F]\right]=-\nabla_{x} W_{\hbar}[F]$. 
By the part (b) of the proof of Proposition B.5 in [10] we know that, for $\|D\|_{1}<\infty$,

$$
\left\|W_{\hbar}[D]\right\|_{\infty} \leq \frac{\|D\|_{1}}{(\pi \hbar)^{d}}
$$

so that, for any $F \in \mathcal{D}(\mathfrak{H})$,

$\|F\|_{1},\left\|\frac{1}{i \hbar}[x, F]\right\|_{1},\left\|\frac{1}{i \hbar}[-i \hbar \nabla, F]\right\|_{1} \leq 1 \Longrightarrow\left\|(\pi \hbar)^{d} W_{\hbar}[F]\right\|_{L^{\infty}}$ and $\operatorname{Lip}\left((\pi \hbar)^{d} W_{\hbar}[F]\right) \leq 1$.

Finally, a straightforward computation shows that, for $\|D\|_{1}<\infty$,

$$
(2 \pi \hbar)^{d}\left\|W_{\hbar}[D]\right\|_{L^{2}}^{2} \leq\|D\|_{2}^{2}, \leq\|D\|_{1}^{2},
$$

so that, for $\frac{\pi \hbar}{2} \leq 1$,

$$
\|D\|_{1} \leq 1 \Longrightarrow\|D\|_{2} \leq\left(\frac{\pi \hbar}{2}\right)^{-\frac{d}{2}} \Longrightarrow\left\|(\pi \hbar)^{d} W_{\hbar}[D]\right\|_{L^{2}\left(\mathbf{R}^{2 d}\right)} \leq 1 .
$$

Therefore,

$\|F\|_{1},\left\|\frac{1}{i \hbar}[x, F]\right\|_{1},\left\|\frac{1}{i \hbar}[-i \hbar \nabla, F]\right\|_{1} \leq 1 \Longrightarrow\left\|(\pi \hbar)^{d} W_{\hbar}[F]\right\|_{L^{2}\left(\mathbf{R}^{2 d}\right)} ;\left\|\nabla(\pi \hbar)^{d} W_{\hbar}[F]\right\|_{L^{2}\left(\mathbf{R}^{2 d}\right)} \leq 1$, and, by (103) and the change of test function $f \rightarrow(\pi \hbar)^{d} W_{\hbar}[F]$, we get $d(\cdot, \cdot) \leq$ $2^{d} \delta\left(W_{\hbar}[\cdot], W_{\hbar}[\cdot]\right)$.

The symmetry in the argument and the triangle inequality for $d$ and $\delta$ are obvious by construction. Moreover $2^{d} \delta\left(W_{\hbar}[\cdot], W_{\hbar}[\cdot]\right) \geq d(\cdot, \cdot)=d_{1}(\cdot, \cdot)$ where $d_{1}$ is the distance defined in (110) below. Therefore $d(\cdot, \cdot)$ and $\delta\left(W_{\hbar}[\cdot], W_{\hbar}[\cdot]\right)$ separate points.

Proposition B.3. Let $R, S \in \mathcal{D}^{2}(\mathfrak{H})$, with Wigner transforms $W_{\hbar}[R], W_{\hbar}[S]$ and Husimi transforms $\widetilde{W}_{\hbar}[R], \widetilde{W}_{\hbar}[S]$. Then

$$
\begin{aligned}
\delta\left(W_{\hbar}[R], W_{\hbar}[S]\right) & \leq \operatorname{dist}_{\mathrm{MK}, 2}\left(\widetilde{W}_{\hbar}[R], \widetilde{W}_{\hbar}[S]\right)+\sqrt{\hbar}\left\|W_{\hbar}[R]-W_{\hbar}[S]\right\|_{L^{2}\left(\mathbf{R}^{2 d}\right)} \\
& =\operatorname{dist}_{\mathrm{MK}, 2}\left(\widetilde{W}_{\hbar}[R], \widetilde{W}_{\hbar}[S]\right)+\sqrt{\hbar} \frac{\|R-S\|_{2}}{(2 \pi \hbar)^{d / 2}}
\end{aligned}
$$

Proof. Using successively formulas (7.1) and formula (7.3) in chapter 7 of [19], we have

$$
\left|\int f(x, \xi)\left(\widetilde{W}_{\hbar}[R]-\widetilde{W}_{\hbar}[S]\right)(x, \xi) d x d \xi\right| \leq \operatorname{Lip}(f) \operatorname{dist}_{M K, 2}\left(\widetilde{W}_{\hbar}[R], \widetilde{W}_{\hbar}[S]\right)
$$

Since $\widetilde{W}_{\hbar}=e^{\hbar \Delta / 4} W_{\hbar}$ one find

$$
\begin{aligned}
\left|\int f(x, \xi)\left(W_{\hbar}[R]-W_{\hbar}[S]\right)(x, \xi) d x d \xi\right| & -\operatorname{Lip}(f) \operatorname{dist}_{\mathrm{MK}, 2}\left(\widetilde{W}_{\hbar}[R], \widetilde{W}_{\hbar}[S]\right) \\
& \leq\left|\int\left(e^{\hbar \Delta / 4}-1\right) f(x, \xi)\left(W_{\hbar}[R]-W_{\hbar}[S]\right)(x, \xi) d x d \xi\right| \\
& \leq\left\|\left(e^{\hbar \Delta / 4}-1\right) f\right\|_{L^{2}\left(\mathbf{R}^{2 d}\right)}\left\|W_{\hbar}[R]-W_{\hbar}[S]\right\|_{L^{2}\left(\mathbf{R}^{2 d}\right)}
\end{aligned}
$$

by Cauchy-Schwarz.

By $\left(e^{\hbar \Delta / 4}-1\right)^{2}=\left(e^{\hbar \Delta / 2}-1\right)-2\left(e^{\hbar \Delta / 4}-1\right)$ and $1-e^{\hbar \Delta / \lambda} \leq-\hbar \Delta / \lambda, \lambda>0$, one gets

$$
\left\|\left(e^{\hbar \Delta / 4}-1\right) f\right\|_{L^{2}\left(\mathbf{R}^{2 d}\right)} \leq \sqrt{\hbar}\|\nabla f\|_{L^{2}\left(\mathbf{R}^{2 d}\right)}
$$

which gives the desired inequality. 
Let $R(t)$ solves the von Neumann equation (7) with initial condition $R^{i n}$ and let $\Phi^{t}$ be the underlying Hamiltonian flow of Hamiltonian $\frac{1}{2} p^{2}+V(q)$ as defined by (12). Let moreover $\mathcal{R}(t)$ be defined by

$$
W_{\hbar}[\mathcal{R}(t)]=W_{\hbar}\left[R^{i n}\right] \circ \Phi^{-t}
$$

Note that, since $R^{i n}$ is Hilbert-Schmidt and $\Phi^{-t}$ is symplectic, $W_{\hbar}[\mathcal{R}(t)]$ is square integrable on $\mathbf{R}^{2 d}$ so that $\mathcal{R}(t)$ is well defined as a Hilbert-Schmidt operator.

Moreover, at the contrary of $\widetilde{W}_{\hbar}[\mathcal{R}(t)]$ which is, as $\mathcal{R}(t)$, not positive, $\widetilde{W}_{\hbar}\left[R^{i n}\right] \circ \Phi^{-t}$ is a probability measure since $R^{i n}$ is positive (and $\Phi^{-t}$ symplectic).

Theorem B.4. Let $V \in C^{1,1}$. Then

$$
\begin{aligned}
& \delta\left(W_{\hbar}[R(t)], W_{\hbar}[\mathcal{R}(t)]\right) \\
\leq & \operatorname{dist}_{\mathrm{MK}, 2}\left(\widetilde{W}_{\hbar}[R(t)], \widetilde{W}_{\hbar}\left[R^{i n}\right] \circ \Phi^{-t}\right)+\sqrt{\hbar}\left(1+e^{1+\operatorname{Lip}(\nabla V))|t|)}\right)\left\|W_{\hbar}\left[R^{i n}\right]\right\|_{L^{2}} \\
= & \operatorname{dist}_{\mathrm{MK}, 2}\left(\widetilde{W}_{\hbar}[R(t)], \widetilde{W}_{\hbar}\left[R^{i n}\right] \circ \Phi^{-t}\right)+\sqrt{\hbar}\left(1+e^{(1+\operatorname{Lip}(\nabla V)|t|)}\right)\left\|R^{i n}\right\|_{2} /(2 \pi \hbar)^{d / 2}
\end{aligned}
$$

Proof. One starts again with

$\left.\left|\iint f(x, \xi)\left(\widetilde{W}_{\hbar}[R(t)]-\widetilde{W}_{\hbar}\left[R^{i n}\right] \circ \Phi^{-t}\right)(x, \xi) d x d \xi\right| \leq \operatorname{Lip}(f) \operatorname{dist}_{\mathrm{MK}, 2}\left(\widetilde{W}_{\hbar}[R], \widetilde{W}_{\hbar}[S] \circ \Phi^{-t}\right)\right)$

Since $\Phi^{t}$ preserves the measure on phase space we get

$$
\begin{aligned}
\int f(x, \xi) \widetilde{W}_{\hbar}\left[R^{i n}\right] \circ \Phi^{-t}(x, \xi) d x d \xi & =\int f \circ \Phi^{t}(x, \xi) \widetilde{W}_{\hbar}\left[R^{i n}\right](x, \xi) d x d \xi \\
& =\int\left(e^{\hbar \Delta / 4}\left(f \circ \Phi^{t}\right)\right)(x, \xi) W_{\hbar}\left[R^{i n}\right](x, \xi) d x d \xi \\
& =\int f(x, \xi)\left(W_{\hbar}\left[R^{i n}\right] \circ \Phi^{-t}\right)(x, \xi) d x d \xi \\
& +\int g^{t}(x, \xi) W_{\hbar}\left[R^{i n}\right](x, \xi) d x d \xi
\end{aligned}
$$

with

$$
g^{t}(x, \xi)=\left(e^{\hbar \Delta / 4}-1\right)\left(f \circ \Phi^{t}\right)(x, \xi)
$$

Therefore

$$
\begin{aligned}
\left|\iint f\left(W_{\hbar}[R(t)]-W_{\hbar}\left[R^{i n}\right] \circ \Phi^{-t}\right) d x d \xi\right| & \leq \operatorname{Lip}(f) \operatorname{dist}_{\mathrm{MK}, 2}\left(\widetilde{W}_{\hbar}[R(t)], \widetilde{W}_{\hbar}[S] \circ \Phi^{-t}\right) \\
& +\int\left(g^{t=0} W_{\hbar}[R(t)]-g^{t} W_{\hbar}\left[R^{i n}\right]\right)(x, \xi) d x d \xi \\
& \leq\left\|g^{t=0}\right\|_{L^{2}}\left\|W_{\hbar}[R(t)]\right\|_{L^{2}}+\left\|g^{t}\right\|_{L^{2}}\left\|W_{\hbar}\left[R^{i n}\right]\right\|_{L^{2}} \\
& =\left(\left\|g^{t=0}\right\|_{L^{2}}+\left\|g^{t}\right\|_{L^{2}}\right)\left\|W_{\hbar}\left[R^{i n}\right]\right\|_{L^{2}}
\end{aligned}
$$

Since $g^{t=0}=\left(e^{\hbar \Delta / 4}-1\right) f$, the first term in the parenthesis in (108) can be estimated by (106). The second one will be treated by the following result. 
Lemma B.5. Let $\Phi^{t}$ the Hamiltonian flow associated to $\frac{1}{2} p^{2}+V(q)$ with $V \in C^{1,1}$. Then, for all $t \in \mathbf{R}$ and all $f \in W^{1,2}$, and all $1 \leq p<\infty$,

$$
\left\|d \Phi^{t}\right\|_{L^{\infty}} \leq e^{(1+\operatorname{Lip}(\nabla V)|t|)} \quad \text { and } \quad\left\|\nabla\left(f \circ \Phi^{t}\right)\right\|_{L^{p}} \leq e^{(1+\operatorname{Lip}(\nabla V)|t|)}\|\nabla f\|_{L^{p}} .
$$

Proof. By the Lipschitz condition on $V$ we have, for all $z, z^{\prime} \in \mathbf{R}^{2 d}$,

$$
\begin{aligned}
\left|\Phi^{t}(z)-\Phi^{s}\left(z^{\prime}\right)\right| & =\| z-z^{\prime} \mid+\int_{0}^{t} \partial_{s}\left(\Phi^{t}(z)-\Phi^{s}\left(z^{\prime}\right)\right) d s \\
& \leq|| z-z^{\prime}\left|+(1+\operatorname{Lip}(\nabla V)) \int_{0}^{t}\right| \Phi^{s}(z)-\Phi^{s}\left(z^{\prime}\right) \mid d s
\end{aligned}
$$

so that, by the Gronwall Lemma, $\Phi^{t}$ is Lipschitz for all $t$ with Lipschtz constant smaller than $e^{(1+\operatorname{Lip}(\nabla V))|t|)}$. Therefore, $\mathrm{d} \Phi^{t}$ exists a.e., $\left\|\mathrm{d} \Phi^{t}\right\|_{L^{\infty}} \leq e^{(1+\operatorname{Lip}(\nabla V)|t|)}$. Moreover $\left|\nabla\left(f \circ \Phi^{t}\right)\right| \leq e^{(1+\operatorname{Lip}(\nabla V)|t|)}\left|(\nabla f) \circ \Phi^{t}\right|$ a.e. and we can perform the change of variable $z \rightarrow \Phi^{t}(z)$ in $\left\|(\nabla f) \circ \Phi^{t} \mid\right\|_{L^{2}}$. Since, being a symplectomorphism, $\Phi^{t}$ preserves the Lebesgue measure on phase space, we get the second inequality in Lemma B.5.

The end of the proof of the first inequality in Theorem B.4 is achieved by (106) and Lemma B.5. The second one follows from the first one by (104).

\section{B.2. $L^{\infty}$ test functions.}

Definition B.6. Let $R, S \in \mathcal{D}(\mathfrak{H}) \cup\left\{\mathcal{R} \in \mathcal{D}_{H S}(\mathfrak{H}), W_{\hbar}[\mathcal{R}] \in L^{1}\left(\mathbf{R}^{2 d}\right)\right\}$, with Wigner transforms $W_{\hbar}[R], W_{\hbar}[S]$. We define, for any integer $M>0$,

$$
\text { (109) } \delta_{M}\left(W_{\hbar}[R], W_{\hbar}[S]\right):=\max _{|\alpha|,|\beta| \leq M}\left\|\partial_{x}^{\alpha} \partial_{\xi}^{\beta} f\right\|_{L^{\infty} \leq 1}\left|\int\left(W_{\hbar}[R]-W_{\hbar}[S]\right)(x, \xi) f(x, \xi) d x d \xi\right| .
$$

and

$$
d_{M}(R, S):=\max _{|\alpha|,|\beta| \leq M} \sup _{\mid \mathcal{D}_{-i \hbar \nabla}^{\alpha} \mathcal{D}_{x}^{\beta} F \|_{1} \leq 1}|\operatorname{trace}(F(R-S))|,
$$

where $\mathcal{D}_{A}=\frac{1}{i \hbar}[A, \cdot]$ for each (possibly unbounded) self-adjoint operator $A$ on $\mathfrak{H}$.

Note that when $R \in \mathcal{D}(\mathfrak{H}),|\operatorname{trace} F R| \leq\|F\|_{\infty}\|R\|_{1} \leq 1$ and when $R \in \mathcal{D}_{H S}(\mathfrak{H})$, $|\operatorname{trace} F R| \leq(F, R)_{H S} \leq\|R\|_{H S}\|F\|_{H S} \leq\|R\|_{H S}\|F\|_{1} \leq\|R\|_{H S}<\infty$, so that $d<\infty$.

Moreover, when $M \geq[d / 2]+2, \max _{|\alpha|,|\beta| \leq M}\left\|\partial_{x}^{\alpha} \partial_{\xi}^{\beta} f\right\|_{L^{\infty}} \leq 1$ implies by the CalderonVaillancourt theorem that $f$ is the Wigner function of a bounded operator $F$ such that $\|F\| \leq \gamma_{d}(2 \pi \hbar)^{d}$. Therefore, when $R \in \mathcal{D}(\mathfrak{H}) \int W_{\hbar}[R] f(x, \xi) d x d \xi=(2 \pi \hbar)^{-d}$ trace $R F \leq$ $\gamma_{d}<\infty$, and when $W_{\hbar}[R] \in L^{1}\left(\mathbf{R}^{2 d}\right), \iint W_{\hbar}[R] f(x, \xi) d x d \xi \leq\left\|W_{\hbar}[R]\right\|_{1}<\infty$, so that $\delta_{M}<\infty, M \geq[d / 2]+2$.

By the same proof than the one of Lemma B2 in [10] one sees that $d_{M}$ is a distance, and by the same argument as for $\delta$ in the proof of Proposition B.2, together with the first inequality in Proposition B.7 below, one sees that $\delta_{M}$, considered as a functions of Wigner functions is also a distance. 
In order to use the estimate of $\gamma_{d}$ proved in the next section, we will restrict ourselves to the following restriction (because $2[d / 4]+2<[d / 2]+2$ ) of Proposition B5 in [10].

Proposition B.7. Let $R, S \in \mathcal{D}_{H S}(\mathfrak{H})$. Then

$$
d_{2[d / 4]+3}(R, S) \leq 2^{d} \delta_{2[d / 4]+3}\left(W_{\hbar}[R], W_{\hbar}[S]\right) .
$$

Moreover, if $R, S$ are density operators, then

$$
\delta_{2[d / 4]+3}\left(W_{\hbar}[R], W_{\hbar}[S]\right) \leq \operatorname{dist}_{\mathrm{MK}, 2}\left(\widetilde{W}_{\hbar}[R], \widetilde{W}_{\hbar}[S]\right)+\frac{2 d \gamma_{d}}{\sqrt{\pi}} \sqrt{\hbar}
$$

where $\gamma_{d}$ is the constant that appears in Theorem C.4 below.

Let us remark that $\delta_{M}$ is decreasing with respect to $M$, so the second inequality is valid for any $\delta_{M}, M \geq 2[d / 4]+3$.

Proof. The second inequality (for $M=[d / 2]+2$ but the proof is insensitive to $M$ ) is contained in Proposition B5 in [10], together with the first one in the case of density operators. It is easy to check that the proof of the latter extends verbatim to the Hilbert-Schmidt case, as it use only the fact that $W_{\hbar}[R-S]$ is square integrable.

The following result is the analogue of Theorem B.4 in Section B.2 above.

Let $R(t)$ solves the von Neumann equation (7) with initial condition $R^{i n}$ and let $\Phi^{t}$ be the underlying Hamiltonian flow of Hamiltonian $\frac{1}{2} p^{2}+V(q)$ as defined by (12).

Theorem B.8. Let $V \in C^{1,1}$ and $W_{\hbar}\left[R^{i n}\right] \in L^{1}\left(\mathbf{R}^{2 d}\right)$. Then

$$
\begin{aligned}
\delta_{2[d / 4]+3}\left(W_{\hbar}[R(t)], W_{\hbar}\left[R^{i n}\right] \circ \Phi^{t}\right) & \leq \operatorname{dist}_{\mathrm{MK}, 2}\left(\widetilde{W}_{\hbar}[R(t)], \widetilde{W}_{\hbar}\left[R^{i n}\right] \circ \Phi^{-t}\right) \\
& +\sqrt{\hbar} \frac{d}{\pi}\left(\gamma_{d}+e^{1+\operatorname{Lip}(\nabla V))|t|)}\left\|W_{\hbar}\left[R^{i n}\right]\right\|_{L^{1}\left(\mathbf{R}^{2 d}\right)}\right)
\end{aligned}
$$

Proof. One again one starts with

$\left.\left|\iint f(x, \xi)\left(\widetilde{W}_{\hbar}[R(t)]-\widetilde{W}_{\hbar}\left[R^{i n}\right] \circ \Phi^{-t}\right)(x, \xi) d x d \xi\right| \leq \operatorname{Lip}(f) \operatorname{dist}_{\mathrm{MK}, 2}\left(\widetilde{W}_{\hbar}[R], \widetilde{W}_{\hbar}[S] \circ \Phi^{-t}\right)\right)$ The term involving $\int f \widetilde{W}[R(t)]$ is treated along the same lines as for the beginning of the proof of theorem B.3 and we get that

$$
\left|\int f \widetilde{W}[R(t)] d x d \xi-\int f W[R(t)]\right| d x d \xi \leq \sqrt{\hbar} \frac{d \gamma_{d}}{\sqrt{2 \pi}} .
$$

Indeed, by the definition (115), (116) and Theorem C.4 in Appendix C below,

$$
\begin{aligned}
\left|\int f(\widetilde{W}[R]-W[R]) d x d \xi\right| & =\left|\int\left(e^{\hbar \Delta / 4}-1\right) f W[R] d x d \xi\right| \\
& =\left|\operatorname{trace}\left(\mathrm{Op}_{\hbar}^{W}\left(\left(e^{\hbar \Delta / 4}-1\right) f\right) R\right)\right| \\
& \leq\left\|\mathrm{Op}_{\hbar}^{W}\left(\left(e^{\hbar \Delta / 4}-1\right) f\right)\right\||\operatorname{trace} R| \\
& \leq \gamma_{d} \max _{|\alpha|,|\beta| \leq 2[d / 4]+2}\left|D_{x}^{\alpha} D_{\xi}^{\beta}\left(e^{\hbar \Delta / 4}-1\right) f\right| \\
& \leq \sqrt{\hbar} \frac{d \gamma_{d}}{\pi} \max _{|\alpha|,|\beta| \leq 2[d / 4]+3}\left|D_{x}^{\alpha} D_{\xi}^{\beta} f\right|
\end{aligned}
$$


For the second term we write, denoting $z=(x, \xi), z^{\prime}+\left(x^{\prime}, \xi^{\prime}\right)$,

$$
\begin{aligned}
\int f \widetilde{W}\left[R^{i n}\right] \circ \Phi^{-t} d x d \xi & =(\pi \hbar)^{-d} \int f(z) e^{-\left(\Phi^{-t}(z)-z^{\prime}\right)^{2} / \hbar} W\left[R^{i n}\right]\left(z^{\prime}\right) d z d z^{\prime} \\
& =(\pi \hbar)^{-d} \int f\left(\Phi^{t}(z)\right) e^{-\left(z-z^{\prime}\right)^{2} / \hbar} W\left[R^{i n}\right]\left(z^{\prime}\right) d z d z^{\prime} \\
& =\int\left(e^{\hbar \Delta / 4}\left(f \circ \Phi^{t}\right)\right)\left(z^{\prime}\right) W\left[R^{i n}\right]\left(z^{\prime}\right) d z^{\prime} \\
& =\int\left(f \circ \Phi^{t}-g_{t}\right)\left(z^{\prime}\right) W\left[R^{i n}\right]\left(z^{\prime}\right) d z^{\prime} \\
& =\int f\left(W\left[R^{i n}\right] \circ \Phi^{-t}\right) d x d \xi-\int g_{t} W\left[R^{i n}\right] d x d \xi
\end{aligned}
$$

with

$$
\begin{aligned}
g_{t}(x, \xi) & =f \circ \Phi^{t}(x, \xi)-e^{\hbar \Delta / 4}\left(f \circ \Phi^{t}\right)(x, \xi) \\
& =\int\left(f \circ \Phi^{t}(x+\sqrt{\hbar} q, \xi+\sqrt{\hbar} p)-f \circ \Phi^{t}(x, \xi)\right) e^{-|q|^{2}-|p|^{2}} d q d p / \pi^{d} .
\end{aligned}
$$

By Lemma B.5,

$$
\left|g_{t}(x, \xi)\right| \leq \sqrt{\hbar}\left\|d \phi^{t}\right\|_{L^{\infty}} \frac{d}{\pi} \operatorname{Lip}(f) . \leq \sqrt{\hbar} e^{1+\operatorname{Lip}(\nabla V))|t|)} \frac{d}{\pi} .
$$

and therefore

$$
\begin{aligned}
\left|\int f \widetilde{W}\left[R^{i n}\right] \circ \Phi^{t}-\int f W\left[R^{i n}\right] \circ \Phi^{t}\right| d x d \xi & \leq\left|-\int g_{t} W\left[R^{i n}\right]\right| d x d \xi \\
& \leq \sqrt{\hbar} \frac{d}{\pi} e^{1+\operatorname{Lip}(\nabla V))|t|)}\left\|W_{\hbar}\left[R^{i n}\right]\right\|_{L^{1}\left(\mathbf{R}^{2 d}\right)} .
\end{aligned}
$$

We conclude by adding (111) and (113).

Let us define finally

$$
\delta_{M, 1}\left(W_{\hbar}[R], W_{\hbar}[S]\right):=\sup _{\substack{f \in L^{2}\left(\mathbf{R}^{2 d}\right) \\ \max _{|\alpha|,|\beta| \leq M}\left\|\partial_{x}^{\alpha} \partial_{\xi}^{\beta} f\right\|_{L^{\infty}} \leq 1 \\\|\nabla f\|_{L^{1}\left(\mathbf{R}^{2 d}\right)} \leq 1}}\left|\int\left(W_{\hbar}[R](x, \xi)-W_{\hbar}[S](x, \xi)\right) f(x, \xi) d x d \xi\right| .
$$

Theorem B.9. Let $V \in C^{1,1}$ and $W_{\hbar}\left[R^{i n}\right] \in L^{\infty}\left(\mathbf{R}^{2 d}\right)$. Then

$$
\begin{aligned}
\delta_{2[d / 4]+3,1}\left(W_{\hbar}[R(t)], W_{\hbar}\left[R^{i n}\right] \circ \Phi^{t}\right) & \leq \operatorname{dist}_{\mathrm{MK}, 2}\left(\widetilde{W}_{\hbar}[R(t)], \widetilde{W}_{\hbar}\left[R^{i n}\right] \circ \Phi^{t}\right) \\
& +\sqrt{\hbar}\left(\frac{d \gamma_{d}}{\pi}+e^{1+\operatorname{Lip}(\nabla V))|t|)}\left\|W_{\hbar}\left[R^{i n}\right]\right\|_{L^{\infty}\left(\mathbf{R}^{2 d}\right)}\right)
\end{aligned}
$$

Proof. The proof is quasi identical to the one of Theorem B.8. the only change consists in estimating the last integral in (112) by

$$
\left|\int g_{t} W\left[R^{i n}\right] d x d \xi\right| \leq\left\|W\left[R^{i n}\right]\right\|_{L^{\infty}\left(\mathbf{R}^{2 d}\right)}\left\|g_{t}\right\|_{L^{1}\left(\mathbf{R}^{2 d}\right)}
$$


We estimate $\left\|g_{t}\right\|_{L^{1}\left(\mathbf{R}^{2 d}\right)}$ by

$$
\begin{aligned}
\int\left|g_{t}(x, \xi)\right| d x d \xi & =\int\left|f \circ \Phi^{t}(x, \xi)-e^{\hbar \Delta / 4}\left(f \circ \Phi^{t}\right)(x, \xi)\right| d x d \xi \\
& =\int\left|\left(f \circ \Phi^{t}(x+\sqrt{\hbar} q, \xi+\sqrt{\hbar} p)-f \circ \Phi^{t}(x, \xi)\right) e^{-|q|^{2}-|p|^{2}}\right| d q d p d x d \xi / \pi^{d} \\
& \leq\left\|\nabla\left(f \circ \Phi^{t}\right)\right\|_{L^{1}\left(\mathbf{R}^{2 d}\right)} \leq\left\|d \Phi^{t}\right\|_{L^{\infty}\left(\mathbf{R}^{2 d}\right)}\|\nabla f\|_{L^{1}\left(\mathbf{R}^{2 d}\right)} .
\end{aligned}
$$

And we conclude by Lemma B.5 in the $L^{1}$ version,

\section{Appendix C. An estimation of the Calderon-Vaillancourt constant}

In this section we revisit the proof of the Caderon-Vaillancourt Theorem by keeping track of the constants. We follow the proof by Hwang [14], as presented in [15].

Let us remind first the definition of the Weyl quantization (see [7] for extensive details).

To $a \in \mathcal{S}\left(\mathbf{R}^{2 d}\right)$ we associate the operator $\mathrm{Op}_{\hbar}^{W}(a)$ on $L^{2}\left(\mathbf{R}^{d}\right)$ defined by its integral kernel given by

$$
\mathrm{Op}_{\hbar}^{W}(a)(x, y):=\int_{\mathbf{R}^{d}} a\left(\frac{x+y}{2}, \xi\right) e^{i \frac{(x-y) \xi}{\hbar}} \frac{d \xi}{(2 \pi \hbar)^{d}} .
$$

A link with Wigner functions can be expressed by the (easily checkable) following identity, valid e.g. for $\mathrm{Op}_{\hbar}^{W}(a)$ bounded and $R$ trace class

$$
\operatorname{trace}\left(\mathrm{Op}_{\hbar}^{W}(a) E\right)=\int_{\mathbf{R}^{2 d}} a(x, \xi) W_{\hbar}[R](x, \xi) d x d \xi
$$

Finally, defining $a_{\hbar}(x, \xi):=a(x, \hbar \xi)$ we immediatly get that

$$
\mathrm{Op}_{\hbar}^{W}(a)=\left(O p_{1}^{W}\left(a_{\hbar}\right):=a_{\hbar}(x, D)\right. \text {. }
$$

We first define, for $k \in \mathbf{N}, k>[d / 2], k$ even, that is $k=2[d / 4]+2, x \in \mathbf{R}^{d}$,

$$
P_{k}(x)=\left(1+|x|^{2}\right)^{k / 2}
$$

and, for $u \in L^{2}$,

Obviously

$$
W_{u}(x, \xi)=\int u(y) P_{k}(x-y)^{-1} e^{-i y \xi} d y
$$

$$
\left\|W_{u}\right\|_{L^{2}} \leq\|u\|_{L^{2}}\left\|P_{k}^{-1}\right\|_{L^{2}}:=C_{k}\|u\|_{L^{2}}
$$

\section{Lemma C.1.}

$$
C_{k}^{2}=\int_{\mathbf{R}^{d}} \frac{1}{\left(1+x^{2}\right)^{k}} d x \leq 2 \frac{\operatorname{vol}\left(S_{d-1}\right)}{2 k-d}=\frac{2 \pi^{\frac{d}{2}}}{\Gamma(d / 2+1)(2 k-d)}
$$

Note that $C_{k}<\infty$ only for $k>d / 2$.

Proof.

$$
\int_{0}^{1} \frac{\rho^{d-1}}{\left(1+\rho^{2}\right)^{k}} d \rho \leq \int_{0}^{1} \rho^{d-1} d \rho=d^{-1}, \int_{1}^{\infty} \frac{\rho^{d-1}}{\left(1+\rho^{2}\right)^{k}} d \rho \leq \int_{1}^{\infty} \rho^{d-1-2 k} d \rho=(2 k-d)^{-1}
$$


Moreover, for all $\alpha,|\alpha| \leq k$, and calling $D:=-i \nabla$,

$$
\left\|D^{\alpha} P_{k}^{-1}\right\|_{L^{\infty}} \leq C_{\alpha, k} .
$$

\section{Lemma C.2.}

$$
C_{\alpha, k} \leq \sum_{m=0}^{|\alpha|-1} k^{|\alpha|-m}(3|\alpha|)^{m}|\alpha|^{d} \leq|\alpha|^{d} k^{|\alpha|}(3|\alpha| / k)^{|\alpha|}=|\alpha|^{d}(3|\alpha|)^{|\alpha|} .
$$

Proof. One first remark that, for $|\beta|=1$,

$$
D^{\beta} P_{k}(x)^{-1}=k \frac{x^{\beta}}{1+x^{2}} P_{k}(x)^{-1}
$$

Therefore the highest term in $k$ in $D^{\alpha} P_{k}^{-1}$ will be

$$
k^{|\alpha|} \sum_{\left|\alpha^{\prime}\right|=|\alpha|} \frac{x^{\alpha^{\prime}}}{\left(1+x^{2}\right)^{\left|\alpha^{\prime}\right|}} P_{k}(x)^{-1} \leq k^{|\alpha|}|\alpha|^{d}
$$

The preceding term will be

$$
k^{|\alpha|-1} \sum_{\left|\alpha^{\prime}\right|=|\alpha|-1}\left(D^{\alpha-\alpha^{\prime}} \frac{x^{\alpha^{\prime}}}{\left(1+x^{2}\right)^{\left|\alpha^{\prime}\right|}}\right) P_{k}(x)^{-1} \leq k^{|\alpha|-1}|\alpha|^{d} 3|\alpha|
$$

since, for $|\beta|=1$,

$$
\begin{aligned}
\left|D^{\beta} \frac{x^{\alpha^{\prime}}}{\left(1+x^{2}\right)^{\left|\alpha^{\prime}\right|}}\right| & =\left|\frac{D^{\beta} x^{\alpha^{\prime}}}{\left(1+x^{2}\right)^{\left|\alpha^{\prime}\right|}}-2\right| \alpha^{\prime}\left|\frac{x^{\alpha^{\prime}} x^{\beta}}{\left(1+x^{2}\right)^{\left|\alpha^{\prime}\right|+1}}\right| \\
& =\left|\beta \cdot \alpha^{\prime} \frac{x^{\alpha^{\prime}-\beta}}{\left(1+x^{2}\right)^{\left|\alpha^{\prime}\right|}}-2\right| \alpha^{\prime}\left|\frac{x^{\alpha^{\prime}} x^{\beta}}{\left(1+x^{2}\right)^{\left|\alpha^{\prime}\right|+1}}\right| \leq 3|\alpha|,
\end{aligned}
$$

due to,

$$
\frac{\left|x_{1}^{\alpha_{1}} \ldots x_{m}^{\alpha_{m}}\right|}{\left(1+x^{2}\right)^{M}} \leq \frac{\left|x_{1}\right|^{\alpha_{1}}}{\left(1+x^{2}\right)^{\alpha_{i}}} \cdots \frac{\left|x_{m}\right|^{\alpha_{m}}}{\left(1+x^{2}\right)^{\alpha_{m}}} \leq 1,\left|\alpha_{1}+\cdots+\alpha_{m}\right| \leq M
$$

The next term will be

$$
k^{|\alpha|-2} \sum_{\left|\alpha^{\prime \prime}\right|=|\alpha|-2}\left(D^{\alpha-\alpha^{\prime \prime}} \frac{x^{\alpha^{\prime \prime}}}{\left(1+x^{2}\right)^{\left|\alpha^{\prime \prime}\right|}}\right) P_{k}(x)^{-1} \leq k^{|\alpha|-2}(|\alpha|-2)^{d} d^{2} 3|\alpha|^{2} \leq k^{|\alpha|-2} 3^{2}|\alpha|^{2}|\alpha|^{d}
$$

since, decomposing $\alpha-\alpha^{\prime \prime}=\beta+\beta^{\prime},|\beta|=\left|\beta^{\prime}\right|=1$,

$$
\begin{aligned}
\mid D^{\beta^{\prime}} D^{\beta} \frac{x^{\alpha^{\prime \prime}}}{\left(1+x^{2}\right)^{\left|\alpha^{\prime \prime}\right|} \mid=} & \left|\beta \cdot \alpha^{\prime \prime} D^{\beta^{\prime}} \frac{x^{\alpha^{\prime \prime}-\beta}}{\left(1+x^{2}\right)^{\left|\alpha^{\prime \prime}\right|}}-2\right| \alpha^{\prime \prime}\left|D^{\beta^{\prime}} \frac{x^{\alpha^{\prime \prime}} x^{\beta}}{\left(1+x^{2}\right)^{\left|\alpha^{\prime \prime}\right|+1}}\right| \\
= & \left|\left(\beta \cdot \alpha^{\prime \prime}\right)\left(\beta^{\prime} \cdot\left(\alpha^{\prime \prime}-\beta\right)\right) \frac{x^{\alpha^{\prime \prime}-\beta-\beta^{\prime}}}{\left(1+x^{2}\right)^{\left|\alpha^{\prime \prime}\right|}}-2\right| \alpha^{\prime \prime} \mid \beta \cdot \alpha^{\prime \prime} \frac{x^{\alpha^{\prime \prime}-\beta+\beta^{\prime}}}{\left(1+x^{2}\right)^{\left|\alpha^{\prime \prime}\right|+1}} \\
& -2\left|\alpha^{\prime \prime}\right| \beta^{\prime} \cdot\left(\alpha^{\prime \prime}+\beta\right) \frac{x^{\alpha^{\prime \prime}+\beta-\beta^{\prime}}}{\left(1+x^{2}\right)^{\left|\alpha^{\prime \prime}\right|+1}}+4\left|\alpha^{\prime \prime}\right|\left(\left|\alpha^{\prime \prime}\right|+1\right) \frac{x^{\alpha^{\prime \prime}+\beta+\beta^{\prime}}}{\left(1+x^{2}\right)^{\left|\alpha^{\prime \prime}\right|+2} \mid} \\
\leq & 9\left|\alpha^{\prime \prime}\right|^{2} \leq 9|\alpha|^{2}=3^{2}|\alpha|^{2}
\end{aligned}
$$


Obviously, for $\left|\beta_{l}\right|=1, l=1, \ldots, m$ and $\left|\alpha^{\prime}\right| \leq|\alpha|$,

$$
\left|D^{\beta_{m}} D^{\beta_{m-1}} D^{\beta_{1}} \frac{x^{\alpha^{\prime}}}{\left(1+x^{2}\right)^{\left|\alpha^{\prime}\right|}}\right| \leq 3^{m}|\alpha|^{m}
$$

and the $m$ th preceding term will be estimated by

$$
k^{|\alpha|-m}(3|\alpha|)^{m}|\alpha|^{d} .
$$

We turn now to the proof of the Calderon-Vaillancourt Theorem in the framework of Weyl quantization. In order to lighten the fiormulas, we will perform the computation in the homogeneous (non semiclassical) case $\hbar=1$ and get back to the semiclassical situation thanks to (117).

Denoting now by $\hat{v}=(2 \pi)^{-d} \int_{\mathbf{R}^{d}} v(y) e^{i \xi y} d y$ the (renormalized and non unitary) Fourier transform of $u$, we first note that

$$
e^{i \xi x} v(x)=P_{k}\left(D_{x}\right)\left(W_{\hat{v}}(\xi, x) e^{i x \xi}\right) .
$$

We get, for $a(x, \xi) k$ times differentiable,

$$
\begin{aligned}
(2 \pi)^{d}(\bar{u}, a(x, D) v) & =\int a\left(\frac{x+y}{2}, \xi\right) e^{i \xi(x-y)} u(y) v(x) d x d y d \xi \\
& =\int a\left(\frac{x+y}{2}, \xi\right) P_{k}^{-1}(x-y) P_{k}\left(D_{\xi}\right) e^{i \xi(x-y)} u(y) v(x) d x d y d \xi \\
& =\int\left(\int u(y) P_{k}^{-1}(x-y) e^{-i \xi y} P_{k}\left(D_{\xi}\right) a\left(\frac{x+y}{2}, \xi\right) d y\right) e^{i \xi x} v(x) d x d \xi \\
& =\int W_{u P_{k}\left(D_{\xi}\right) a\left(\frac{x+.}{2} \cdot \xi\right)}(x, \xi) e^{i x \xi} v(x) d x d \xi \\
& =\int W_{u P_{k}\left(D_{\xi}\right) a\left(\frac{x+.}{2} \cdot \xi\right)}(x, \xi) P_{k}\left(D_{x}\right)\left(W_{\hat{v}}(\xi, x) e^{i x \xi}\right) d x d \xi \\
& =\int P_{k}\left(D_{x}\right)\left(W_{u P_{k}\left(D_{\xi}\right) a\left(\frac{x+.}{2} \cdot \xi\right)}(x, \xi)\right) W_{\hat{v}}(\xi, x) e^{i x \xi} d x d \xi
\end{aligned}
$$

since, by (119), $P_{k}^{-1}$ is $k$ times differentiable (as well as, let us recall, $a(x, \xi)$ ).

Therefore

$$
(2 \pi)^{d}|(\bar{u}, a(x, D) v)| \leq\left\|P_{k}\left(D_{x}\right) W_{u P_{k}\left(D_{\xi}\right) a\left(\frac{x+\cdot}{2} . \xi\right)}(x, \xi)\right\|_{L^{2}\left(\mathbf{R}^{2 d}\right)}\left\|W_{\hat{v}}(\xi, x)\right\|_{L^{2}\left(\mathbf{R}^{2 d}\right)} .
$$

We know that, by (118),

$$
\left\|W_{\hat{v}}\right\|_{L^{2}} \leq C_{k}\|v\|_{L^{2}}
$$

Moreover, for $l \in \mathbf{N}$,

$$
\left(D_{x}^{2}\right)^{l}\left(W_{u P_{k}\left(D_{\xi}\right) a\left(\frac{x+.}{2} . \xi\right)}(x, \xi)\right)=\sum_{|\alpha+\beta| \leq 2 l} c_{\alpha, \beta}^{l} \int u(y) P_{k}\left(D_{\xi}\right) D_{x}^{\alpha} a\left(\frac{x+y}{2} \cdot \xi\right) D_{x}^{\beta} P_{k}(x-y)^{-1} e^{-i y \xi} d y
$$


Indeed

$$
\left(D_{x}^{2}\right)^{l}=\sum_{l_{1}+\cdots+l_{d}=l}\left(\begin{array}{c}
l \\
\left(l_{1}, \ldots, l_{d}\right)
\end{array}\right) \prod_{i=1}^{d} D_{x_{i}}^{2 l_{i}} .
$$

where the multinomial coefficient $\left(\begin{array}{c}l \\ \left(l_{1}, \ldots, l_{d}\right)\end{array}\right)$ is defined by

$$
\left(\sum_{i=1}^{d} x_{i}\right)^{l}=\sum_{l_{1}+\ldots l_{d}=l}\left(\begin{array}{c}
l \\
\left(l_{1}, \ldots, l_{d}\right)
\end{array}\right) \prod_{i=1}^{m} x_{i}^{l_{i}} .
$$

In particular

$$
\sum_{l_{1}+\ldots l_{d}=l}\left(\begin{array}{c}
l \\
\left(l_{1}, \ldots, l_{d}\right)
\end{array}\right)=d^{l}
$$

We have

$$
\begin{aligned}
& D_{x_{i}}^{2 l_{i}}\left(W_{u P_{k}\left(D_{\xi}\right) a\left(\frac{x+\cdot}{2} \cdot \xi\right)}(x, \xi)\right) \\
= & D_{x_{i}}^{2 l_{i}} \int u(y) P_{k}\left(D_{\xi}\right)\left(a\left(\frac{x+y}{2}, \xi\right)\right) P_{k}(x-y)^{-1} e^{-i y \xi} d y \\
= & \int \sum_{m_{i}=1}^{2 l_{i}}\left(\begin{array}{c}
2 l_{i} \\
m_{1}
\end{array}\right) D_{x_{i}}^{2 l_{i}-m_{i}}\left(P_{k}\left(D_{\xi}\right) a\left(\frac{x+y}{2}, \xi\right)\right) D_{x_{i}}^{m_{i}}\left(P_{k}^{-1}(x-y)\right) u(y) e^{-i \xi y} d y
\end{aligned}
$$

so that

$$
\begin{aligned}
& \left(D_{x}^{2}\right)^{l}\left(W_{u P_{k}\left(D_{\xi}\right) a\left(\frac{x+\cdot}{2} . \xi\right)}(x, \xi)\right) \\
= & \int \sum_{l_{1}+\cdots+l_{d}=l}\left(\begin{array}{c}
l \\
\left(l_{1}, \ldots, l_{d}\right)
\end{array}\right) \prod_{i=1}^{d} \sum_{m_{i}=1}^{2 l_{i}}\left(\begin{array}{c}
2 l_{i} \\
m_{1}
\end{array}\right) D_{x_{i}}^{2 l_{i}-m_{i}} P_{k}\left(D_{\xi}\right) a\left(\frac{x+y}{2}, \xi\right) D_{x_{i}}^{m_{i}} P_{k}^{-1}(x-y) u(y) e^{-i \xi y} d y \\
= & \int \sum_{l_{1}+\cdots+l_{d}=l}\left(\begin{array}{c}
l \\
\left(l_{1}, \ldots, l_{d}\right)
\end{array}\right) \prod_{i=1}^{d} \sum_{\alpha_{i}+\beta_{i}=2 l_{i}} D_{x_{i}}^{\alpha_{i}} P_{k}\left(D_{\xi}\right) a\left(\frac{x+y}{2}, \xi\right) D_{x_{i}}^{\beta_{i}} P_{k}^{-1}(x-y) u(y) e^{-i \xi y} d y \\
= & \int \sum_{\substack{|\alpha+\beta|=2 l \\
\alpha_{i}+\beta_{i} \text { even }}}\left(\begin{array}{c}
\left(\frac{\alpha_{1}+\beta_{i}}{2}, \ldots, \frac{\alpha_{d}+\beta_{d}}{2}\right) \\
\hdashline
\end{array}\right)\left(\begin{array}{c}
\alpha+\beta \\
\beta
\end{array}\right) \prod_{i=1}^{d} D_{x_{i}}^{\alpha_{i}} P_{k}\left(D_{\xi}\right) a\left(\frac{x+y}{2}, \xi\right) D_{x_{i}}^{\beta_{i}} P_{k}^{-1}(x-y) u(y) e^{-i \xi y} \\
= & \int \sum_{\substack{|\alpha+\beta|=2 l \\
\alpha_{i}+\beta_{i} \text { even }}} c_{\alpha, \beta}^{l} \prod_{i=1}^{d} D_{x_{i}}^{\alpha_{i}} P_{k}\left(D_{\xi}\right) a\left(\frac{x+y}{2}, \xi\right) D_{x_{i}}^{\beta_{i}} P_{k}^{-1}(x-y) u(y) e^{-i \xi y} d y
\end{aligned}
$$

with

$$
c_{\alpha, \beta}^{l}=\left(\begin{array}{c}
l \\
\left(\frac{\alpha_{1}+\beta_{1}}{2}, \ldots, \frac{\alpha_{d}+\beta_{d}}{2}\right)
\end{array}\right)\left(\begin{array}{c}
\alpha+\beta \\
\beta
\end{array}\right)
$$


Lemma C.3.

Proof. Just observe that $\left(\begin{array}{c}\alpha+\beta \\ \beta\end{array}\right) \leq 2^{|\alpha+\beta|} \leq 2^{2 l}$.

$$
c_{\alpha, \beta}^{l} \leq 2^{2 l}\left(\begin{array}{c}
l \\
\left(\frac{\alpha_{1}+\beta_{i}}{2}, \ldots, \frac{\alpha_{d}+\beta_{d}}{2}\right)
\end{array}\right) .
$$

Therefore, developing $P_{k}\left(D_{x}\right)$ and $P_{k}\left(D_{\xi}\right)$,

$$
\begin{aligned}
& P_{k}\left(D_{x}\right)\left(W_{u P_{k}\left(D_{\xi}\right) a\left(\frac{x+\cdot}{2} \cdot \xi\right)}(x, \xi)\right) \\
= & \sum_{l, l^{\prime}=1}^{k / 2}\left(\begin{array}{l}
k \\
l
\end{array}\right)\left(\begin{array}{c}
k \\
l^{\prime}
\end{array}\right) \sum_{\substack{|\alpha+\beta|=2 l \\
\alpha_{i}+\beta_{i} \text { even }}} c_{\alpha, \beta}^{l} \int u(y)\left(D_{\xi}^{2}\right)^{l^{\prime}} D_{x}^{\alpha} a\left(\frac{x+y}{2} \cdot \xi\right) D_{x}^{\beta} P_{k}(x-y)^{-1} e^{-i y \xi} d y,
\end{aligned}
$$

and, using (118),

$$
\begin{aligned}
& \left\|P_{k}\left(D_{x}\right)\left(W_{u P_{k}\left(D_{\xi}\right) a\left(\frac{x+\cdot}{2} \cdot \xi\right)}(x, \xi)\right)\right\|_{L^{2}} \\
& \leq \sum_{l, l^{\prime}=1}^{k / 2}\left(\begin{array}{l}
k \\
l
\end{array}\right)\left(\begin{array}{l}
k \\
l^{\prime}
\end{array}\right) \sum_{\substack{|\alpha+\beta|=2 l \\
\alpha_{i}+\beta_{i} \text { even }}} c_{\alpha, \beta}^{l}\left\|u\left(D_{\xi}^{2}\right)^{l^{\prime}} D_{x}^{\alpha} a\left(\frac{x+\cdot}{2} \cdot \xi\right)\right\|_{L^{2}}\left\|D_{x}^{\beta} P_{k}^{-1}\right\|_{L^{2}} \\
& \leq\|u\|_{L^{2}} \sum_{l, l^{\prime}=1}^{k / 2}\left(\begin{array}{l}
k \\
l
\end{array}\right)\left(\begin{array}{l}
k \\
l^{\prime}
\end{array}\right) \sum_{\substack{|\alpha+\beta|=2 l \\
\alpha_{i}+\beta_{i} \text { even }}} C_{\beta, k} \max _{\substack{l^{\prime} \leq k \\
|\alpha| \leq k}}\left\|\left(D_{\xi}^{2}\right)^{l^{\prime}} D_{x}^{\alpha} a\right\|_{L^{\infty}} \\
& :=C^{k} \max _{\substack{l^{\prime} \leq k / 2 \\
|\alpha| \leq k}}\left\|\left(D_{\xi}^{2}\right)^{l^{\prime}} D_{x}^{\alpha} a\right\|_{L^{\infty}}\|u\|_{L^{2} .}
\end{aligned}
$$

with, by Lemma C.3 and Lemma C.2

$$
\begin{aligned}
C^{k} & =\sum_{l, l^{\prime}=1}^{k / 2}\left(\begin{array}{l}
k \\
l
\end{array}\right)\left(\begin{array}{c}
k \\
l^{\prime}
\end{array}\right) \sum_{\substack{|\alpha+\beta|=2 l \\
\alpha_{i}+\beta_{i} \text { even }}} c_{\alpha, \beta}^{l} C_{\beta, k} \\
& \leq 2^{k} \sum_{\substack { l, l^{\prime}=1 \\
\begin{subarray}{c}{|\alpha+\beta|=2 l \\
\alpha_{i}+\beta_{i} \text { even }{ l , l ^ { \prime } = 1 \\
\begin{subarray} { c } { | \alpha + \beta | = 2 l \\
\alpha _ { i } + \beta _ { i } \text { even } } }\end{subarray}} c_{\alpha, \beta}^{l}|\beta|^{d}(3|\beta|)^{|\beta|} \\
& \leq \frac{k^{2}}{4} 2^{k} k^{d}(3 k)^{k} \sum_{\substack{|\alpha+\beta|=2 l \\
\alpha_{i}+\beta_{i} \text { even }}} c_{\alpha, \beta}^{l} \\
& \leq \frac{k^{2}}{4} 2^{k} k^{d}(3 k)^{k} 2^{2 k} \sum_{k_{1}+\cdots+k_{d}=k}\left(\begin{array}{c}
k \\
\left(k_{1}, \ldots, k_{d}\right)
\end{array}\right) \\
& \leq \frac{k^{2}}{4} 2^{k} k^{d}(3 k)^{k} 2^{2 k} d^{k}=\frac{k^{2} 2^{3 k}}{4}(3 k)^{k} k^{d} d^{k}
\end{aligned}
$$


by (122).

Finally, by (120), (121) and (123),

$$
|(\bar{u}, a(x, D) v)| \leq D_{k} \max _{\substack{l^{\prime} \leq k / 2 \\|\alpha| \leq k}}\left\|\left(D_{\xi}^{2}\right)^{l^{\prime}} D_{x}^{\alpha} a\right\|_{L^{\infty}}\|u\|_{L^{2}}\|v\|_{L^{2}}
$$

with, by (121),

$$
D_{k}^{d}:=(2 \pi)^{-d} C^{k} C_{k}=(2 \pi)^{-d} \frac{\sqrt{2} \pi^{-d / 4} k^{2} 2^{3 k}}{4 \sqrt{\Gamma(d / 2+1)(2 k-d)}}(3 k)^{k} k^{d} d^{k}
$$

for any integer $k$ such that $2 k>d$.

Therefore, in particular,

$$
\left.\|a(x, D)\| \leq D_{2[d / 4]+2}^{d} \max _{\substack{l^{\prime} \leq 2[d / 4]+2 \\|\alpha| \leq 2[d / 4]+2}} \|\left(D_{\xi}^{2}\right)^{l^{\prime}}\right) D_{x}^{\alpha} a \|_{L^{\infty}}
$$

and, e.g., for $d \geq 4$ (so that $2[d / 4]+2 \leq d$ ),

We just proved the following result

$$
D_{2[d / 4]+2} \leq \frac{d^{3 / 4}\left(192 e^{-\frac{1}{4}} \pi^{-\frac{5}{4}}\right)^{d}}{4 e^{\frac{1}{4}}}\left(d^{d}\right)^{11 / 4} .
$$

Theorem C.4 (Calderon-Vaillancourt).

$$
\|a(x, D)\| \leq \gamma_{d} \max _{\substack{|\beta| \leq 2[d / 4]+2 \\|\alpha| \leq 2[d / 4]+2}}\left\|D_{\xi}^{\beta} D_{x}^{\alpha} a\right\|_{L^{\infty}}
$$

with

$$
\gamma_{d}=D_{2[d / 4]+2}^{d}
$$

The semiclassical result is the same by (117): for $\hbar \leq 1$,

$$
\left\|\mathrm{Op}_{\hbar}^{W}(a)\right\| \leq \gamma_{d} \max _{\substack{|\beta| \leq 2[d / 4]+2 \\|\alpha| \leq 2[d / 4]+2}}\left\|D_{\xi}^{\beta} D_{x}^{\alpha} a\right\|_{L^{\infty}} .
$$

Acknowledgements. The work of Thierry Paul was partly supported by LIA LYSM (co-funded by AMU, CNRS, ECM and INdAM). T.P. thanks also the Dipartimento di Matematica, Sapienza Università di Roma, for its kind hospitality for several stays during the development of this work.

\section{REFERENCES}

[1] L. Ambrosio, A. Figalli, G. Friesecke, J. Giannoulis, T. Paul, Semiclassical limit of quantum dynamics with rough potentials and well posedness of transport equations with measure initial data, Communication on Pure and Applied Mathematics, 64, 1199-1242 2011.

[2] A. Athanassoulis, N. Mauser, T. Paul, Coarse-scale representations and smoothed Wigner transforms, Journal de Mathématiques Pures et Appliquées, 91, p. 296-338 2009.

[3] H. Bacry, A. Grossman, and J. Zak:Proof of completeness of lattice states in the kq representation, Phys. Rev. B 12 (1975), 1118- .

[4] E. Caglioti, F. Golse and T. Paul. Towards quantum transport, preprint ... 
[5] A. Dunford and A. Schwartz, Theory of operators, Addison-Wesley, 1988.

[6] A. Figalli, M. Ligab, T. Paul Semiclassical limit for mixed states with singular and rough potentials, Indiana University Mathematics Journal, 61, (2013), 193-222.

[7] G. Folland, Harmonic Analysis in Phase Space, Annals of Mathematics Studies 122, Princeton University Press 1989.

[8] P.Gérard: Mesures semi-classiques et ondes de Bloch. Seminaire sur les Équations aux Dérivées Partielles, 1990-1991. Exp. No. XVI, 19 pp., École Polytechnique, Palaiseau, 1991.

[9] P.Gérard, P.A.Markowich, N.J.Mauser, F.Poupaud: Homogenization limits and Wigner transforms. Comm. Pure Appl. Math. 50 (1997), 323-379.

[10] F. Golse, S. Jin, T. Paul, On the Convergence of Time Splitting Methods for Quantum Dynamics in the Semiclassical Regime, Foundations of Computational Mathematics DOI: 10.1007/s10208-02009470-z.

[11] F. Golse, C. Mouhot, T. Paul, On the Mean-Field and Classical Limits of Quantum Mechanics, Communication in Mathematical Physics 343, 165-205 (2016).

[12] F. Golse, T. Paul: The Schrödinger Equation in the Mean-Field and Semiclassical Regime, Arch. Rational Mech. Anal. 223 (2017), 57-94.

[13] F. Golse, T. Paul: Wave Packets and the Quadratic Monge-Kantorovich Distance in Quantum Mechanics, Comptes Rendus Mathematique 356177 - 197 (2018).

[14] I. L. Hwang, The $L^{2}$-boundedness of pseudodifferential operators, Trans. Amer. Math. Soc.302 (1987), no. $1,5576$.

[15] N. Lerner, Metrics on the Phase Space and Non-Selfadjoint Pseudo-Differential Operators, Birkhäuser 2010.

[16] P.L. Lions, T. Paul, Sur les mesures de Wigner Rev. Mat. Iberoamericana, 9 (1993), 553-618.

[17] Reed, M., Simon, B.: "Methods of Modern Mathematical Physics I. Functional Analysis" Acad. Press., Inc., 1980.

[18] B. Simon: "Trace Ideals and their Applications", 2nd ed., Amer. Math. Soc. Providence, 2005.

[19] C. Villani: "Topics in Optimal Transportation", Amer. Math. Soc., Providence (RI), 2003.

[20] C. Villani: "Optimal Transport. Old and New", Springer-Verlag, Berlin, Heidelberg, 2009.

(F.G.) Ecole polytechnique, CMls, 91128 Palaiseau Cedex, France

E-mail address: francois.golse@polytechnique.edu

(T.P.) CNRS \& LJLL Sorbonne Universit 4 Place Jussieu 75005 Paris, France

E-mail address: thierry.paul@upmc.fr 